\title{
Signal analysis for radiation event identification
}

Andrew C. Stephan ${ }^{* a, b}$, David Bowling ${ }^{c}$, Hee-Jung Im $^{d}$, Sheng Dai ${ }^{d}$, Steven A. Wallace ${ }^{a}$

${ }^{a}$ Neutron Sciences, Inc., Knoxville, TN 37932 USA

${ }^{\mathrm{b}}$ Department of Nuclear Engineering, Pasqua Engineering Building, The University of

Tennessee, Knoxville, TN 37996-2300 USA

${ }^{c}$ Linear Path Consulting, LLC, Knoxville, TN USA

${ }^{\mathrm{d} C h e m i c a l ~ S c i e n c e s ~ D i v i s i o n, ~ M S ~ 6201, ~ O a k ~ R i d g e ~ N a t i o n a l ~ L a b o r a t o r y, ~ P . O . ~ B o x ~ 2008, ~}$ Oak Ridge, TN 37831 USA

\begin{abstract}
An analysis program has been developed to identify scintillation pulses using the digitized signals from two photomultiplier tubes viewing the output from a common source. The analysis is directed towards identifying the noise pulses in the background signal level. In this work a neutron detector test system is constructed and methods incorporated into a program for processing digitized signals from the detector so as to determine the effectiveness of amplitude discrimination for identifying neutron pulses in the presence of spurious events. Two methods are used for identifying pulses, one based on locating a monotonic increase in the signal amplitude exceeding some threshold value and the other on a locating a number of sequential signal data points exceeding the mean and standard deviation of the baseline of the record. The software written for controlling the digital oscilloscope is flexible and allows the selection of records to be taken such that statistically useful properties can be calculated as to the loss of efficiency that results from using a discriminator to remove noise. Experimental results are presented for a lithiated sol-gel based neutron scintillator tested using this system.
\end{abstract}


PACS: 29.85.+c, 29.40.Mc 


\section{Introduction}

The detection of ionizing radiation is an important field with applications in such areas as nuclear medicine, power production, scientific research, and nuclear safeguards and security. Radiation detectors typically have a background signal centered at zero volts with random superimposed pulses produced by radiation detection events and with unwanted noise pulses from other sources. Depending on the type of detector, typical pulses range from a few millivolts to several volts in amplitude and from under a nanosecond to several microseconds in duration. [1] Signals for analysis are either taken directly from the detector or run through a preamp and/or amplifier to achieve desired signal shape characteristics. The two most common pulse shapes encountered are an approximately Gaussian-shaped peak with a lengthened tail and a fast rising pulse followed by an exponentially decaying tail. Both types of pulses may be negative or positive and unipolar or bipolar.

Pulses within a signal may be produced by both radiation detection events and from electronic noise. Many radiation detectors intended to detect a particular type of radiation (e.g. neutrons) are also sensitive to other types of radiation (e.g. gamma rays). [1] Pulse discrimination based on amplitude and/or pulse shape is normally used to distinguish between the real detection events and noise. A major source of electronic noise when using a scintillator in conjunction with a photosensitive detector (e.g. a photomultiplier tube) is radiation interaction inside the photosensitive detector.

A two-dimensional position sensitive neutron detector based on a proprietary lithiated sol-gel scintillator screen and a wavelength-shifting (WLS) optical fiber readout is under evaluation. The crossed fiber method of determining neutron detection location 
is well established. [2-6] The polymerized sol-gel material contains ${ }^{6} \mathrm{Li}$ and fluoresces so that fast pulses of less than 100 nanoseconds are emitted when a neutron absorption occurs. The ${ }^{6} \mathrm{Li}$ absorption produces the emission of an energetic alpha and a triton particle. The present work stems from the need to characterize the lithiated sol-gel material for its sensitivity to gamma radiation and noise relative to the $\mathrm{ZnS}: \mathrm{Ag} /{ }^{6} \mathrm{LiF}$ scintillator material used in prior work. The after glow and long scintillation pulse duration of $\mathrm{ZnS}: \mathrm{Ag}$ result in loss of data when detecting diffracted neutrons at high count rates, providing an impetus to seek alternative scintillators. The purpose of using the lithiated sol-gel scintillator in a WLS-based detector is to allow pulse acquisition and processing of neutrons at rates of $1 \mathrm{MHz}$ or higher per anode.

In WLS-based position sensitive detector systems, pulses must appear in both the $\mathrm{x}$ and the $\mathrm{y}$ position readout signals for a neutron count to be registered and its location determined. The test setup for sol-gel scintillator characterization consists of two photomultiplier tubes (PMTs) between which the scintillator sample is placed, thus giving two signals to be analyzed simultaneously. Since noise from gamma events in the PMTs only affects one detector in each instance, a requirement of coincident pulses in the two signals voids these noise events. Coincident pulses are accepted as neutrons if their amplitudes exceed some threshold value for filtering out gamma events. Each neutron event must be stamped with the location and time of arrival of the neutron. The development efforts with the dual PMT system yielded empirical means of analyzing the signals to identify pulses present in the digitized signals and discriminate noise. These methods include the duration of a consistent pattern of signal value changes, the duration of the signal remaining outside a window centered on the average signal value with 
bounds set by the standard deviation, the total magnitude of the signal value change within one of these periods of change, and the polarity (positive or negative) of the signal value change. This paper discusses the methods used; their application to typical detector pulses, including the strengths and drawbacks of different analysis methods; provides suggestions for adapting the analysis methods to different situations; and presents data for a lithiated sol-gel neutron scintillator.

\section{Hardware and Software Setup}

The detector and data acquisition setup consists of a neutron scintillator as a radiation detection element, two Hamamatsu R580 photomultiplier tubes (PMTs) for conversion of the light emission by the neutron scintillator into an electronic signal, a LeCroy LT372 digitizing oscilloscope for signal capture and download, and a PC to receive the signal communications from the oscilloscope and perform signal storage and analysis. Scintillator samples are chemically synthesized within an O-ring mounted on a PMMA disk that is then seated against the faces of two PMTs. The PMTs are held in a cardboard tube light-proof housing. A diagram of this setup is shown in Figure 1.

Details of the electronics are as follows. The commercial tube base for the R580 PMT was modified to give a direct output, bypassing the built-in preamp. The output signals from the two PMTs are input into channels one and two of the digital oscilloscope with input set to $50 \Omega$ impedance to match the impedance of the cable (the alternative, 10 $\mathrm{M} \Omega$, caused very serious ringing). A computer program was written to allow remote control of the oscilloscope from a personal computer, including setting appropriate data acquisition parameters (e.g. time base, sample size, vertical division value) for the scintillators tested and the automation of data acquisition over long periods of time. The 
oscilloscope is set to immediately begin capturing a new set of data points while downloading the previous set to the computer. In practice the download speed to the computer over the IEEE 488 connection was insufficient to keep pace with the rate at which the oscilloscope acquired data when the event rate was high. In a full detector implementation, high-speed data acquisition systems will use comparators to test 64 lines from the base of a H7546 multianode photomultiplier. The present setup is used to establish the gain needed to maximize the counting of real pulses while utilizing amplitude discrimination to minimize noise.

In a WLS fiber readout scheme, two sets of parallel fibers are used with the two sets oriented perpendicularly to each other. Each set gives the radiation interaction location in one dimension with the event location determined according to the signals from the fibers in that set; the fiber carrying the largest number of photons from the event normally being closest to the event. Each fiber in a set is mapped onto a single cathode of a multi-anode PMT. Signals from the anodes may be logged for off-line analysis or a resistive divider network may be used to divide the signal up between the two ends of the divider strings to give an indication of the event location. [2,3] A scintillator/fiber geometry suitable for this purpose is shown in Figure 2. In our development setup, two PMTs take the place during signal analysis methods development of the multi-anode PMTs that read out the fiber sets. (The multi-anode PMT case differs only from the two PMT case in the number of signals to analyze; the basic methods for discriminating legitimate events from background and noise are the same.) Sol-gel neutron scintillator samples were tested using the dual PMT setup. Pulses produced by the PMT were unipolar with a comparatively fast rise time and slower decay time. 
Custom software was written to automate control of the oscilloscope for sequential capture and download of large numbers of traces, each consisting of many data

points. A separate program is used to analyze the data points in each trace to identify legitimate neutron capture events within a background containing noise and unwanted gamma ray interaction events. Data from both the sol-gel neutron scintillator/dual PMT setup and some additional neutron detectors, such as a surface barrier detector (SBD) coated with lithiated sol-gel glass, were processed using the custom computer code. Both programs are available for download and released under the GNU GPL license. [7]

\section{Waveform Analysis Methods}

A digital oscilloscope is used in the characterization study to record the two input channels at a preset rate. The majority of data is taken with an analog-to-digital conversion every nanosecond. The oscilloscope holds in memory a snapshot of the number of samples selected whenever a digitized 8-bit data point value exceeds a trigger level. For the positive biased photomultipliers used, the scintillation pulses are negative and unipolar. A very useful property of this method of recording the occurrence of pulses is that a baseline is established by retaining in the snapshot the record of the baseline present prior to the pulse exceeding the threshold. (The baseline prior to a pulse was normally quite close to zero with some variation due to electronic noise.) Events, both the desired neutron events and unwanted events such as gamma ray interactions in the scintillator and in the PMT, produce transient pulses. The task is to identify pulses within the signal and extract information concerning their shape and amplitude. Figure 3 shows typical coincident pulses from neutrons as recorded by the experimental setup described above. 
Two methods are employed to identify pulses within the signal. In the first, the program runs through the data points in a record (usually set to 500 samples) to determine their change relative to the previous data point and simultaneously tallies the number of sequential like changes (i.e. all positive or all negative). When the number of sequential like changes exceeds some threshold value, this is taken as an indicator that a pulse occurred. Depending on the polarity of the pulse and the sign (i.e. positive or negative) of the changes, this can be during the rise or fall phase of the pulse. Figure 4 shows an example of how this method works. When tallying the number of sequential changes, the data analysis program assigns sequential falls negative integers and positive integers to sequential rises. Thus, for example, the fifth sequential rise in a series of data points is assigned the value +5 and the fourth fall the value -4 . In addition to a threshold for the number of like sequential changes in the signal value, a second screening method is employed.

The second screening method is to only count such changes as a pulse if at least one of the changes exceeds some minimum value. This is done because the random noise in the signal occasionally results in a significant number of like sequential changes occurring but with a minimal signal value change in each case. The minimum change value must be large enough to be quite unlikely to result from noise while still low enough to catch small (but legitimate) pulses. A value of $0.05 \mathrm{~V}$ was normally used for this purpose.

A flowchart of how like sequential change analysis is accomplished is shown in Figure 5. When Tripped $=$ True and the variable Sequential Counter meets the criteria for 
identifying a pulse (e.g. greater than 4), the current data point is determined to be part of a pulse.

In the second method, the mean and standard deviation of a record are first established and then the data points assessed to determine if they fall outside a window defined by the mean signal value plus and minus two or three times (user selectable) the standard deviation. An example of this is shown in Figure 6. A count is kept in the same fashion as for like sequential changes except that sequential events falling outside the window centered on the mean signal value are tallied instead. When the number of sequential points falling outside the window exceeds a threshold value, a pulse is deemed to have occurred. A simple way of tracking the polarity of a pulse is to assign a negative value to points below the window, a positive value to points above, and a reset of the count to zero when a point either falls within the window or on the opposite side of the window from the previous point.

For both methods, an additional screening method is normally used in which a pulse is only considered legitimate if the absolute value of the farthest outlying point in the pulse exceeds some minimum value. If the user wishes to use a minimum outlier for additional screening against noise, the user may set it to a value that is below that expected of legitimate pulses and above the level for most noise. If most noise is indeed relatively low in amplitude compared to most legitimate neutron pulses, this is very effective in weeding out noise that passes the previous filters.

Finally, very simple FWHM (Full-Width, Half-Max) estimation for pulses is accomplished by determining a base signal level out of which a pulse rises and then counting the number of data points falling between the outlying point in the pulse and 
half-way between the outlying point and the base signal level. Base signal level is taken as the mean plus or minus (as appropriate) two standard deviations for the two standard deviation pulse identification method and the mean plus or minus three standard deviations for the three standard deviation case. In the case of sequential changes, the base level is hard-coded to be near the mean.

\section{Application to Event Identification and Analysis}

Pulses come with a variety of characteristics depending on the type of scintillator detector used, oscilloscope digitization settings, and other variables. Factors that should be considered when selecting a pulse identification and analysis method include the relative noisiness or cleanness of a pulse, the expected duration of a pulse relative to the number of data points in a trace, and the number of pulses in the trace.

The window method based on the mean and the standard deviation is best for pulses that are quite noisy. The reason for this may be seen in Figure 7, where the electronic noise is large compared to the change in signal value from one data point to the next. The result is that only very rarely are there more than two sequential like changes in the signal level, even when the pulse is rising at its fastest rate, and this causes many legitimate pulses to be missed by the sequential changes method. (This problem can sometimes be mitigated by setting the threshold for like sequential changes to a very low value.) By contrast the mean and standard deviation window method is unaffected by noise provided the signal value stays outside the window long enough to indicate a pulse.

If practicable, the signal digitization rate should be selected with the pulse duration in mind. There are several reasons for this. If the digitization rate is very high compared with the pulse duration, noise will predominate over changes in the signal 
value from one point to the next. (The sequential changes method is very sensitive to this but the standard deviation window method is not.) If the digitization rate is too low compared with the pulse duration, some pulses may be missed, accurate information on pulse characteristics such as amplitude and duration will be lost, and pulse analysis to differentiate between legitimate events and noise will become unreliable. For unipolar pulses with fall times that of three or four times the duration of their rise times, it was found that setting the digitization rate to capture a half dozen points during the pulse rise produced good results. The cleaner a pulse is (i.e. the less noise it has), the faster one may digitize without noise predominating over changes in the signal value from one point to the next.

The standard deviation window waveform analysis method is sensitive to pulse duration and the number of pulses in a trace. If pulses are unipolar and comprise a substantial fraction of the total trace duration, the mean value of points in the trace will be skewed in the direction of the polarity of the pulses and the standard deviation will be inflated. If the pulses are bipolar, skewing will be reduced or eliminated but the standard deviation of the trace will be even more affected. Skewing the mean and inflating the standard deviation both tend to raise the bar of what requirements must be met for a pulse to be registered. If the amplitude of the pulses is consistent, this does not represent a difficulty, but if the largest pulses are many times the size of the smallest pulses, small pulses may be missed by the standard deviation window method. In this case, the sequential changes method may alleviate the problem. At high pulse event rates, the sequential changes method also has the advantage of being able to differentiate between 
pulses that partially overlap (pile-up) if set to trip on rise phase of the pulse and if the rise phases of the two pulses do not overlap.

Once a probable pulse event has been identified in a trace, it can be analyzed to determine whether it is a legitimate neutron count or stems from a noise source such as a gamma ray interaction in the PMT. Gamma ray interactions in the PMT produce pulses of much shorter duration (several ns FWHM) than the scintillators we use (FWHM of 10 ns or more). The code includes simple pulse FWHM estimation. Another method for discriminating between legitimate neutron events and noise is to only accept coincident pulses from the two PMTs, the two PMT signals being read in by two separate oscilloscope channels as described in the section on hardware and software setup. Neutron events normally result in pulses from each PMT whereas gamma ray events in a PMT only affect the signal from that PMT. Thus, rejecting non-coincident events eliminates much noise from gammas. Figure 8 shows an example of this.

\section{Pulse Statistics}

A sol-gel glass scintillator doped with ${ }^{6} \mathrm{Li}$ for neutron sensitivity is under evaluation. Testing is conducted at the Spallation Neutron Source (SNS) Californium source at the Oak Ridge National Laboratory (ORNL) Californium Users Facility (CUF). Thirty micrograms of ${ }^{252} \mathrm{Cf}$ are placed beneath $50 \mathrm{~cm}$ of polyethylene and $5 \mathrm{~cm}$ of lead, with the assembly shown in Figure 1 then placed on top. Without the lead brick shielding beneath the PMTs, many gamma pulses as shown in Figure 8 are present. The digital oscilloscope is set to trigger when channel 1 exceeds a preset voltage; thus when an event is captured, there is always a pulse present in this channel. The lack of a pulse in channel 
2 indicates that pulse in channel 1 originates from a particle (most likely a gamma ray) interaction in the PMT connected to channel 1.

Five thousand triggered pulses were analyzed as follows. An event was considered genuine if both channels detected a pulse simultaneously, simultaneity being defined as having points in both channels fall outside the mean $+/$ - three standard deviations window and exceeding $50 \mathrm{mV}$ within a $50 \mathrm{~ns}$ time interval. It was found that $68 \%$ of events were thus considered genuine neutron capture scintillations and $32 \%$ spurious. The ratios of the channel 1 pulse to the channel 2 pulse were computed using the point in each pulse with the highest absolute value. (See Figure 9.) The ratios were distributed between 0.1 and 5 , with a peak around 0.5 . It may be seen from Figure 1 that the efficiency for light collection into the two PMTs will not be the same for both PMTs. The presence of a PMMA disk on one side of the scintillator sample decreases the solid angle subtended by the PMT on that side of the scintillator and thus reduces the number of photons collected in the PMT. The PMMA disk also reduces the number of photons reaching that PMT through attenuation and reflection at material boundaries. The factor of two average difference in average signal amplitude between the PMTs is attributed to these causes.

In addition to using a neutron scintillator with a WLS readout fiber system, if position sensitivity is not necessary (e.g. in a portal monitoring application at a border crossing) the scintillator may be formed into a long cylinder or similar shape with a PMT placed at either end. In this case the significance of the ratio and amplitudes of the pulses in the PMTs at either end of the scintillator may be readily deduced from Figure 10, which shows the variation in signal amplitude from the two PMTs with neutron capture 
location in the scintillator. (The system is idealized with minimal statistical fluctuations in photon generation, direction, and attenuation.) Some minimum pulse amplitude from both PMTs will be required to verify that a neutron detection event has in fact occurred. The amplitude of the signal in each PMT is shown by the exponentially decaying lines that cross in the center of the figure. If the length of the scintillator is too long relative to the light attenuation or the minimum pulse amplitude required for detecting an event too high, portions of the scintillator will be unusable as they will produce a detectable signal in only one (or neither) PMT. These dead zones will initially appear at either end of the scintillator element next to the PMTs and spread inwards as the scintillator length is increased.

\section{Adaptation to Other Applications}

While the methods described are applied specifically to analyzing signal output from a neutron detector, they are broadly applicable to many different signal analysis tasks in which events must be identified within a digitized signal. Such events can be pulses of brief duration or a rise over an extended period of time. In some applications the base signal level is not stable as it is in this work but instead drifts over time. An example of this is identifying temperature transients within a background of drifting ambient temperature.

If drifts in the background signal level occur, the standard deviation window analysis method is most sensitive to them. (The sequential changes method will work if changes are counted only if they exceed a minimum value greater than the maximum expected drift rate.) If the durations of transients are small relative to the timescale over which significant base signal drift occurs, a simple mitigation approach is to slice long 
traces into sections and process each section individually when computing the mean and standard deviation and searching for outlying events as indicators of a pulse. The magnitude of drift in the base signal level will be smaller within each section than across the trace as a whole. If doing this, it is important to make the sections overlap by the typical pulse duration (i.e. some data points are repeated by appearing at the start of one section and the end of another) in case a pulse happens to occur right around the point at which a section division occurs. Checking must be included to make sure such a pulse is not counted twice by being identified in both sections.

Besides its usefulness in reducing the impact of drift of the base signal, slicing signal traces into sections is important for another reason. In a real-time system with a continuous input signal, one cannot wait for the entire signal trace to be captured as data acquisition is always going on. The sequential changes method for pulse identification may be used directly in real-time signal analysis. If the standard deviation window method is used, it will be necessary to divide the captured data into slices based on time to facilitate processing.

\section{Conclusions}

When processing digitized signals from radiation detectors, a method is used to identify legitimate pulses within the signal and avoid spurious events. We have constructed a setup for testing neutron scintillators for use with a crossed fiber readout system and written programs for acquiring and processing data from these scintillators. The signal analysis code employs two basic methods for pulse identification. One involves searching for like sequential changes in the signal value with a possible pulse being identified if the number of like sequential changes exceeds some threshold value. 
The other identifies possible pulses based on the number of sequential data points falling outside a window whose boundaries are a function of the mean value and standard deviation of the signal exceeding some threshold value. Additional screening methods are employed to remove spurious events that are identified by these two methods. The two pulse identification methods have different advantages and disadvantages in different applications.

\section{Acknowledgements}

ACS and SAW were supported by a Small Business Innovation Research (SBIR) Grant from the US Department of Energy (DOE) under Contract DE-FG020101ER83224. H-JI and SD were supported by a CRADA subcontract with Oak Ridge National Laboratory (ORNL) within the SBIR grant. The authors would like to thank Drs. M. Lowell Crow and Ron Cooper for allowing us to use the SNS fixture at the CUF and Cathy Simmons for providing MCNP calculations giving the flux and spectrum of the neutrons passing through the top of the SNS fixture. ORNL is managed for the DOE under contract No. DE-AC05-00OR22725 by UT-Battelle, LLC. Funding of this work by the DOE under the SBIR grant does not constitute an endorsement by the DOE.

\section{References}

[1] G. F. Knoll, Radiation Detection and Measurement, $3^{\text {rd }}$ Edition, Wiley, New York (2000).

[2] D. P. Hutchinson, R. K. Richards, D. E. Holcomb, J. A. Ramsey, "Position sensitive scintillation neutron detectors using a crossed-fiber optic readout array," Proceedings of the SPIE 3769, 88 (1999).

[3] D. P. Hutchinson, R. K. Richards, L. C. Maxey, D. E. Holcomb, R. G. Cooper, "Wavelength-shifting fiber readout of scintillation detectors," presented at the International Workshop on Position-Sensitive Neutron Detectors, June 28-30, 2001, Berlin, Germany. 
[4] T. Adachi, K. Sakai, A. Gorin, K. Kuroda, I. Manuilov, K. Morimoto, T. Oku, A. Ryazantsev, H. M. Shimizu, J. Suzuki, F. Tokanai, "Development of detector based on neutron scintillator with wavelength shifting fiber," Proceedings of the Fifteenth Meeting of the Internal Collaboration on Advanced Neutron Sources 1, 621 (2001).

[5] A. Gorin, K. Kuroda, I. Manuilov, K. Morimoto, T. Oku, A. Ryazantsev, H. M. Shimizu, J. Suzuki, F. Tokanai, "Development of scintillation imaging device for cold neutrons," Nuclear Instruments and Methods A 479, 456 (2002).

[6] K. Toh, M. Katagiri, K. Sakasai, M. Matsubayashi, A. Birumachi, H. Takahashi, M. Nakazawa, "Two-dimensional neutron imaging method using scintillators with wavelength shifting fibers," Nuclear Instruments and Methods A 485, 571 (2002).

[7] http://www.neutronsciences.com/software.html. 


\section{Figure Captions}

Figure 1. Measurement setup for testing scintillator samples.

Figure 2. Operation of a crossed-fiber readout system for neutron detection.

Figure 3. A typical neutron detection event with coincident pulses.

Figure 4. How the sequential like changes method for pulse identification works.

Figure 5. A flowchart showing how data points in a trace are processed to identify like sequential changes.

Figure 6. Pulse identification by sequential events falling outside a window derived from the mean and standard deviation.

Figure 7. A noisy signal where the change in signal value from one data point in the pulse to the next is generally dominated by random noise instead of the pulse shape.

Figure 8 . Noise caused by a gamma ray interacting in one of the PMTs; one signal has a very fast pulse and the other no pulse at all.

Figure 9. Ratio of peak amplitudes (Channel 1/Channel 2) between coincident pulses in the dual PMT test system.

Figure 10. Idealized variation of pulse peak amplitudes with neutron capture location in a scintillator. PMTs are located at opposite ends of the scintillator. The light attenuation factor and minimum pulse amplitude relative to the photon emission intensity of the scintillation event determine the maximum useful scintillator length. 


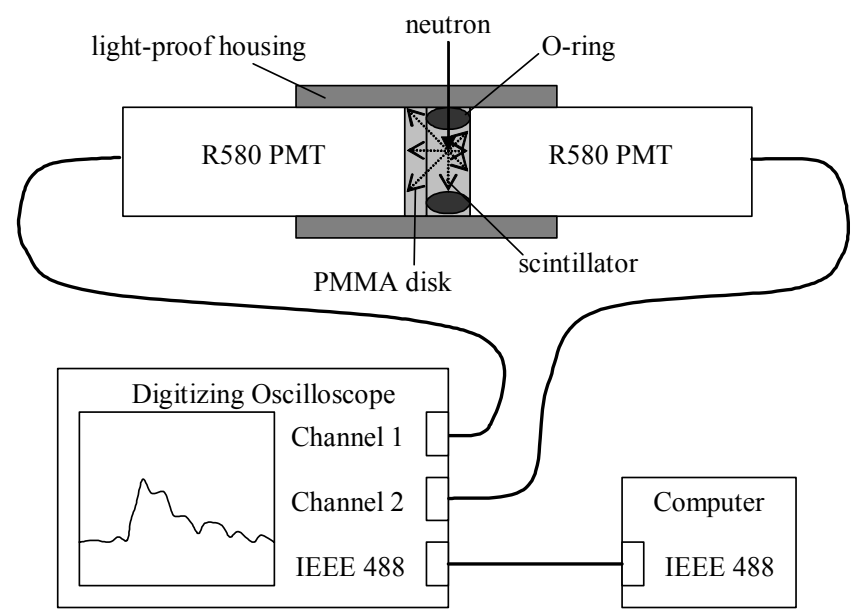

Figure 1. 


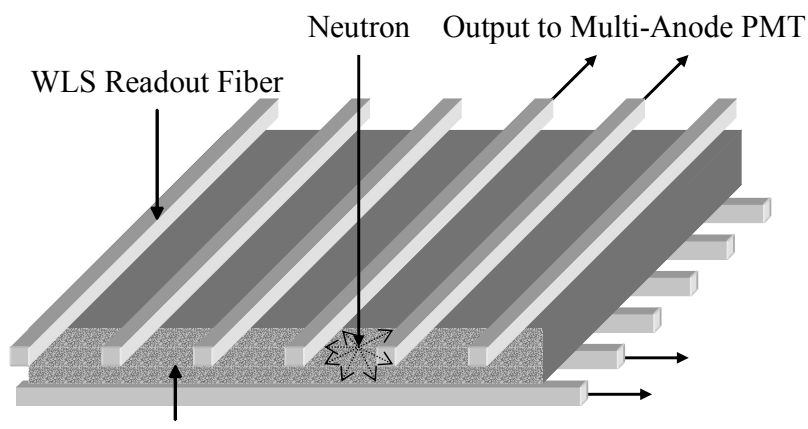

Scintillator Screen

Figure 2. 


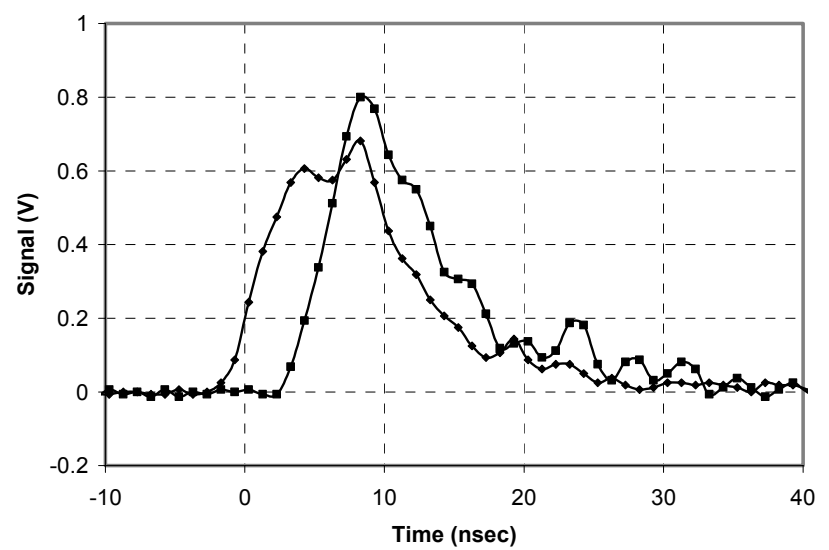

Figure 3. 


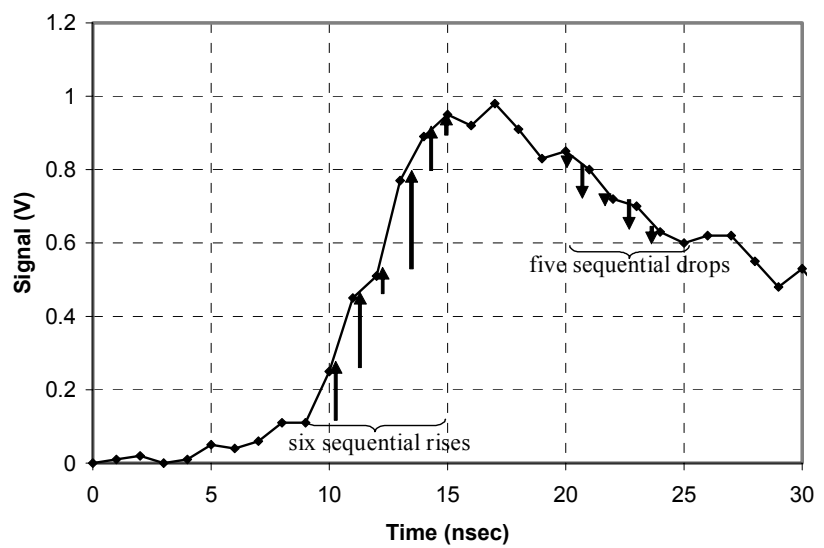

Figure 4. 


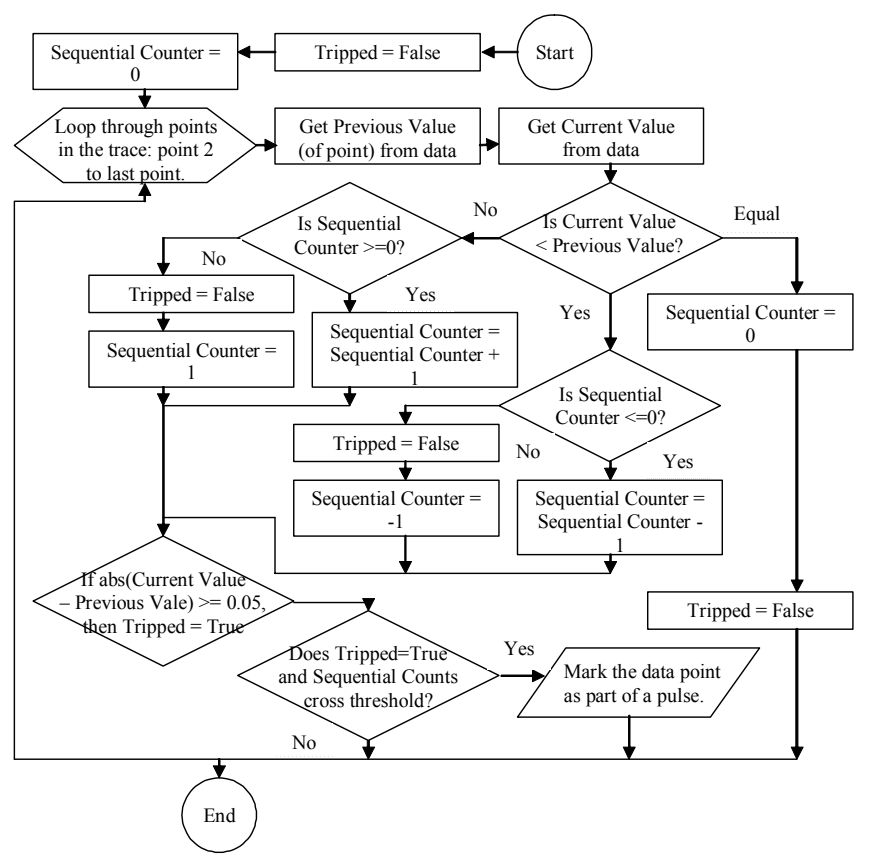

Figure 5. 


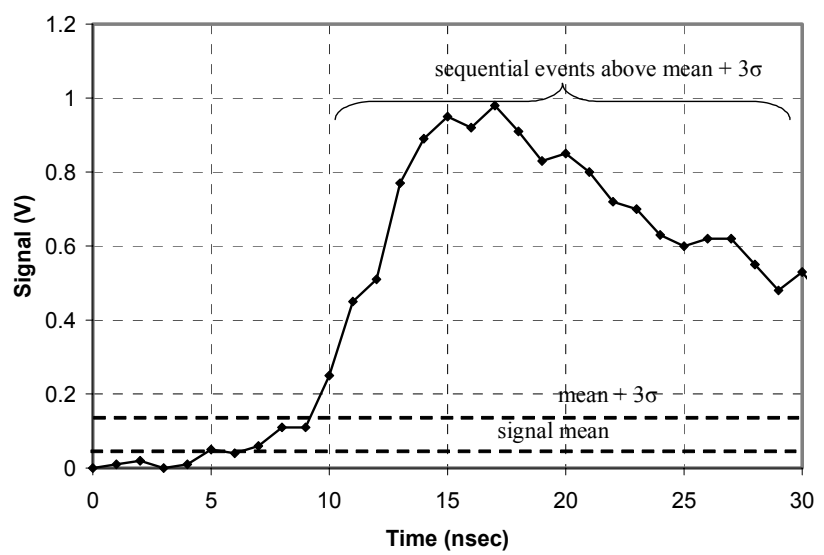

Figure 6. 


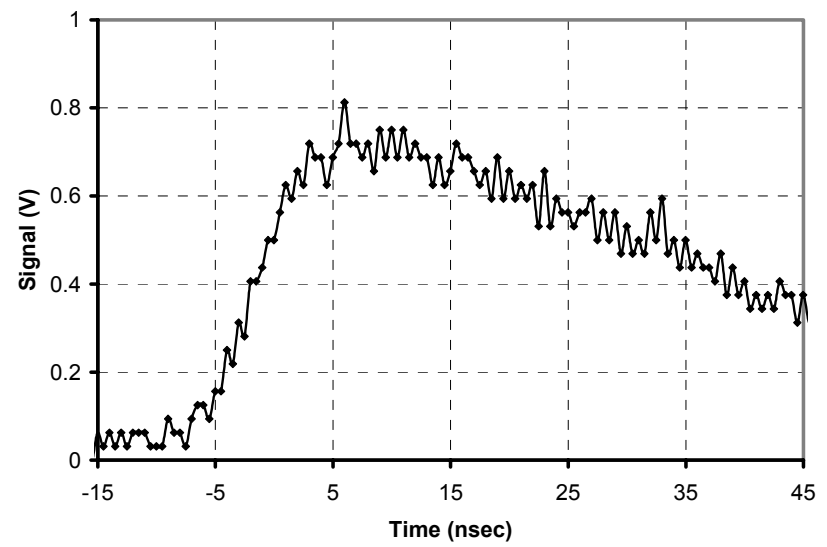

Figure 7. 


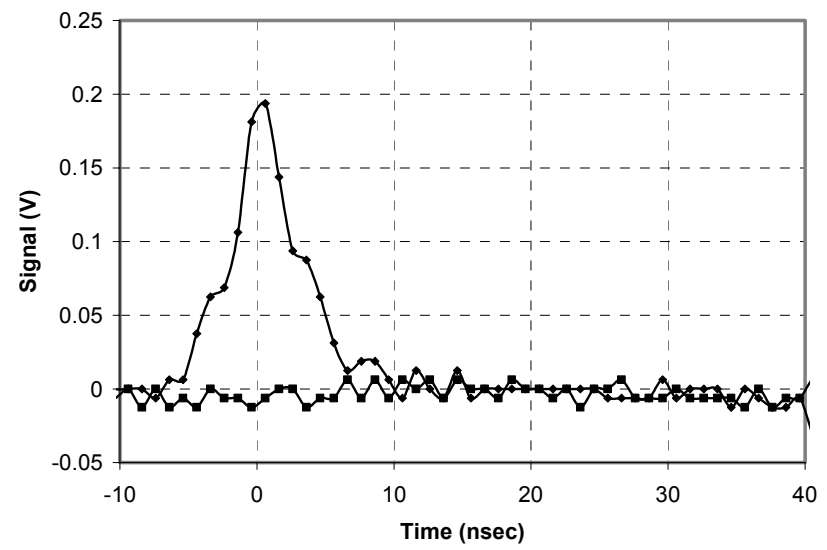

Figure 8 . 


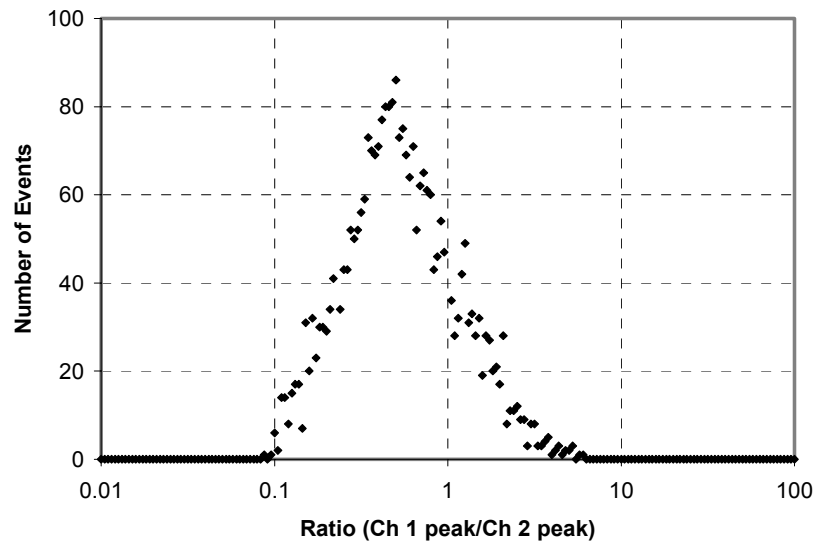

Figure 9. 


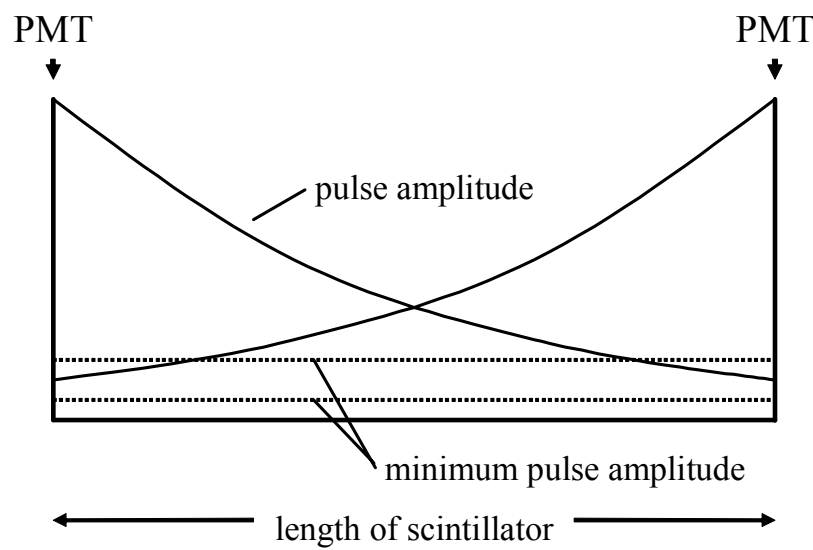

Figure 10. 


\title{
Design considerations for lithiated plastic scintillator systems for SNM
}

\section{detection}

\author{
Andrew C. Stephan ${ }^{1,2}$, Steven A. Wallace ${ }^{*, 1}$, Hee-Jung $\operatorname{Im}^{3}$, Sheng Dai ${ }^{3}$
}

1 - Neutron Sciences, Inc., Knoxville, TN 37932 USA

2 - Department of Nuclear Engineering, University of Tennessee, Knoxville, TN 37996 USA

3 - Chemical Sciences Division, Oak Ridge National Laboratory, Oak Ridge, TN 37831 USA

* - Corresponding author.

\begin{abstract}
Extruded lithiated plastic scintillator bars used with a wavelength-shifting (WLS) fiber readout system provides a simple and inexpensive method for constructing very large volume neutron and high energy gamma detectors for passive scanning and active interrogation for the presence of special nuclear material (SNM). The present work investigates the design of a system based on lithiated plastic for detection of neutrons and high energy gammas. It is shown that detection probabilities for plutonium spontaneous fission neutrons of 0.3 may be readily achieved, double that of the present state-of-theart. Methods are presented for greatly improving neutron-gamma discrimination in lithiated plastic scintillator over simple pulse height analysis methods. Methods for determining stand-off detection distances are developed and stand-off distances calculated for different detector sizes and source cases (stationary source and moving source). Finally, recommendations for future investigations are given.
\end{abstract}


Corresponding author: Steven Wallace, Neutron Sciences, Inc., 1256 Lovell View Drive, Knoxville, TN 37932 USA, telephone: (865) 777-0273, fax: (865) 777-0273, email: swallace@neutronsciences.com.

Keywords: Lithiated plastic scintillator; position-sensitive neutron detection; neutron scintillator detector; neutron scattering research; wavelength-shifting fiber readout PACS Codes: $29.40 . \mathrm{Mc}, 29.40 . \mathrm{Gx}, 28.20 .-\mathrm{v}$ 


\section{Introduction}

As the events of the past several years have demonstrated, terrorism has a global reach and terrorists are seeking increasingly sophisticated methods for the infliction of mass casualties and economic and social disruption. Nuclear terrorism is one of the top sources of concern and the ability to identify and interdict nuclear and radiation weapons is of paramount importance.

Detection of special nuclear material (SNM) of interest to weapons makers is mostly readily accomplished using neutrons or a mixture of neutrons and gamma rays. Neutron emission by an object represents a highly reliable signature of SNM. For example, typical weapons grade plutonium emits 59,100 neutrons $/ \mathrm{kg} . \mathrm{sec}$ via spontaneous fission. [1] Uranium, in contrast to plutonium, has a very low neutron emission rate and its gamma ray emissions are all at low energy where they are easily stopped by any shielding materials.

To effectively detect uranium and to enhance the signal from plutonium and other transuranics for greater detection sensitivity, a variety of active interrogation methods may be used, mostly involving the application of a pulsed neutron flux to a suspect target. If fissile material is present, the neutrons will produce fissions in the material, causing both prompt and delayed fission neutrons and gammas to be released. [2-12] Detection of neutrons during the pulse off phase has been the most commonly used indicator of SNM presence. Investigations have demonstrated that high energy $(>3 \mathrm{MeV})$ delayed gammas are a very robust signature of SNM. High energy gamma rays have the advantage of being emitted in larger numbers than neutrons and being more highly 
penetrating and therefore less effectively shielded by materials surrounding the fissile material (e.g. cargo in a shipping container in which SNM is concealed). [11,12]

The sensitivity of a detector system to radioactive material is a function of its intrinsic efficiency (equivalent to the detection probability; the probability of a radiation particle incident on the detector producing a count in the detector) and the solid angle it subtends around the radioactive material. To maximize sensitivity it is therefore desirable to use a large-area detector with a very high intrinsic detection efficiency, or detection probability. From a practical standpoint, a detector must also be affordable to achieve large-scale deployment at strategic locations such as ports, border crossings, and other key points of transit. For large-area detectors this translates into a low cost per unit detector area or volume.

The present work examines the potential for using lithium-doped plastic scintillator as a neutron and high energy gamma detector in active neutron interrogation systems. For this application, plastic scintillator has the advantages of high detection efficiencies for both neutrons and high energy gammas, low cost, and ruggedness for field use. The scintillator may also be used for passive scanning, particularly for gamma emitters.

High-quality lithiated plastic scintillator is not yet available commercially, although some R\&D work has been reported in the literature using both liquid and plastic bases and efforts to perfect lithiated plastic scintillator are ongoing. [13-16] Considerations concerning lithium and plastic chemistry (hydrophilic lithium is immiscible in hydrophobic plastic) suggest that there is a fundamental limit to the lithium content of lithiated plastic scintillator of perhaps two percent by weight using organic 
lithium salts. This is sufficient to achieve good neutron detection efficiencies, as will be seen.

\section{System Design}

\section{2a. Design Considerations}

There are a number of characteristics that should be exhibited by systems for detecting SNM and other nuclear materials of interest to terrorists. Some, such as ruggedness in the field, are inherent to plastic scintillators. (The photodetector used with the scintillator will typically be the limiting factor for field ruggedness.) Others, such as affordability and ease of deploying a large detection area, depend on how the detection system is designed.

Plastic scintillator may be inexpensively fabricated in very large quantities using existing industrial techniques. [17-20] Lithiated plastic scintillator detects neutrons via the reaction ${ }^{6} \mathrm{Li}\left(\mathrm{n}\right.$, alpha) ${ }^{3} \mathrm{H}, \mathrm{Q}=4.78 \mathrm{MeV} .{ }^{6} \mathrm{Li}$ has a high neutron capture cross section (940 barns at thermal) and consequently strongly absorbs neutrons in the thermal to low epithermal energy range. Although lithiated plastic scintillator will be considerably more expensive than standard plastic scintillator due to the cost of enrichment in the ${ }^{6} \mathrm{Li}$ isotope, enriched neutron target material cost is a major cost driver for neutron detectors generally and does not put lithiated plastic scintillator at a cost disadvantage.

In a full system implementation, the quantity of lithiated plastic scintillator used should be kept at the lowest level that is still consistent with sufficient neutron detection probability. Inexpensive plain plastic scintillator can be used in addition to lithiated plastic scintillator to yield high detection efficiencies for high energy gammas and improve fast neutron detection efficiencies by acting as a neutron moderator and 
reflector, both neutrons and high energy gammas being excellent signatures for the presence of SNM. [12] A reasonable approach is to intersperse bars of lithiated plastic scintillator in with plain plastic scintillator in a pattern that optimizes the detector neutronics while holding down the amount of lithium required.

2b. System Design

A simple and inexpensive readout method is available for large volumes of plastic scintillator extruded as long bars. [21-22] In this method, the bars are coated with lightreflecting material except around a groove running along one side of the bar into which a wavelength-shifting (WLS) readout fiber is placed. Figure 1 shows a picture of two sections of bar with WLS fibers. [23] Scintillation light produced in the bar that enters the WLS fiber is transmitted down the fiber to one anode of a multi-anode photomultiplier tube (MAPMT). The reflective coating on the bars improves the light collection efficiency of the readout fiber and allows each bar to act as an individual detection element independent of other bars. It is a simple matter to deploy large numbers of plastic scintillator bars using only a few MAPMTs and a computer-based signal processing system.

Because scintillation light pulses from individual bars may be read out separately from other bars in this design and from other scintillation pulses in the same bar when the pulses are separated by a short period of time, signal processing methods involving the analysis of pulse patterns may be used to identify particles and particle energies. It should be noted that signal processing is limited by the light collection efficiency of the optical readout fibers; the fibers will only collect a fraction of the total scintillation photons emitted by a bar and the photon collection efficiency will vary with the location 
of the scintillation event within the bar. Further, some light loss will occur in the readout fibers and the fraction of photons lost will depend on how far they must travel through the fiber in order to reach the MAPMT. These factors will cause blurring of the pulse amplitude.

Options do exist for improving light collection in a system based on this detector concept. For example, additional fibers (and fiber grooves) may be added to a bar to improve the number of photons collected from the bar in a pulse event. Detailed investigations of light collection efficiency and design will be left for future investigations.

\section{Monte Carlo Simulation Methods}

\section{3a. Simulation Parameters}

The intent of this work is to examine the response of lithiated plastic scintillator to fast neutrons and gamma rays for detecting special nuclear material (SNM) and other radioactive materials of interest to terrorists. To this end, radiation transport calculations were performed for neutrons and gamma rays over the range 0.1 to $10 \mathrm{MeV}$ using the computer code MCNP for neutrons and MCNP-PoliMi for gammas. [24,25] In MCNP, the ENDF60 cross sections including the poly01.T neutron scattering cross sections were specified and the PTRAC option used to output detailed information on the path and interactions of individual neutrons. The MCNP-PoliMi runs used the IDUM card to generate an output deck detailing the gamma interactions in the scintillator. Runs used

either $10^{5}$ or $2 \times 10^{5}$ particles to produce good statistics. A custom code was written to automate the large number of runs and process their output into a concise form.

\section{3b. Materials Composition}


As mentioned earlier, the lithium content of lithiated plastic scintillators using organic lithium salts is likely limited to two percent by weight due to fundamental chemistry considerations. Doping levels of tenths of a percent by weight have been demonstrated in the literature. $[14,16]$ Accordingly lithium $\left(95 \%\right.$ enriched $\left.{ }^{6} \mathrm{Li}\right)$ levels of $0.1-2 \%$ by weight were used in calculations. Neutronics properties (e.g. neutron capture probability) of the plastic vary considerably over this range, while gamma ray transport and interaction properties are effectively constant, thus gamma data was generated using plastic with $0.5 \%$ lithium. The plastic was assumed to have a 1:1 $\mathrm{H}: \mathrm{C}$ atom ratio and a density of $1 \mathrm{~g} / \mathrm{cm}^{3}$.

\section{3c. Simulation Geometry and Related Assumptions}

Plastic scintillator bars extruded via the existing industrial process are $4.1 \mathrm{~cm}$ wide and $1 \mathrm{~cm}$ thick, with any length desired up to $8 \mathrm{~m}$. Neutron transport and interaction calculations were performed for $0.5 \mathrm{~m}^{2}$ arrays of scintillator bars with thicknesses of 10,20, and $30 \mathrm{~cm}$. (Dimensions were $98.4 \mathrm{~cm}$ x $50 \mathrm{~cm}$ given by 24 bars side-by-side, each $50 \mathrm{~cm}$ long.) Gamma calculations used $1 \mathrm{~m}^{2}$ arrays of scintillator bars with thicknesses of 10,20,30, and $40 \mathrm{~cm}$. (Dimensions were $102.5 \mathrm{~cm}$ x $100 \mathrm{~cm}$ given by 25 bars side-by-side, each $100 \mathrm{~cm}$ long.) In both cases the detector dimensions were such that detection probability losses due to particles escaping out the side were small; thus one would expect that intrinsic detection probability would not change very much were the detectors made larger.

As the WLS fibers are small (mm size scale) and very similar in composition to the plastic, the plastic was modeled as a single block with the dimensions just given. The bars in which individual interactions occurred were determined from the interaction 
location coordinates. It was assumed that charged particles (electrons, alphas, tritons) resulting from gamma and neutron interactions deposit all their energy in the scintillator bar in which they originate. This is a very reasonable assumption given the shortness of the charged particle track lengths compared to the scintillator bar dimensions.

Although gamma and neutron interactions occurring in the readout fibers will produce scintillation pulses, they will be few in number due to the very small volume of the fibers compared to that of the scintillator bars. In an optimized system, one method for discriminating against such events would be to use two or more readout fibers per scintillator bar and only accept events producing light pulses coincidentally in more than one bar.

A system highly optimized for price/performance for detecting neutrons and high energy gammas will contain a mixture of both plain plastic and lithiated plastic scintillator bars. As determining an optimized mix of both types of bars is beyond the scope of this paper, a homogeneous design of all lithiated plastic scintillator bars was used in the calculations.

\section{3d. Scintillation Intensity}

It is well-known that in radiation scintillators the quantum yield for scintillation light emission (light emission per unit of energy deposited) often varies by an order of magnitude or more between different common charged particles (e.g. electrons, protons, alphas) depending on the linear energy transfer (LET) of the particle in question. For this reason, the scintillator light output, measured in $\mathrm{MeV}$ electron equivalent (MeVee), is often used in lieu of the energy deposited in the scintillator by a particle. For this study, we calculated MeVee for different particles as follows: 
For electrons, light_output $=0.99 \cdot E_{e}+0.008, E_{e}>0.0081 \mathrm{MeV} .[26]$

For recoil protons, light_output $=0.0364 \cdot E_{p}^{2}+0.125 \cdot E_{p} \cdot[27]$

For the ${ }^{6} \mathrm{Li}$ neutron capture reaction, light_output $\approx 0.55 \mathrm{MeVee} .[28]$

For fast neutron detection using capture gating following a scintillation pulse from neutron scattering, an LLD of $60 \mathrm{keVee}$ scintillation light emission intensity was used for triggering a start pulse. This corresponds to an energy deposition of around 500 $\mathrm{keV}$ by the neutron.

\section{Results and Discussion: Neutron Detection}

\section{4a. Types of Neutron Interaction Patterns}

As lithiated plastic scintillator is composed almost entirely of hydrogen and carbon atoms, its scattering cross section for neutrons over the full energy range from fission is relatively large. The ${ }^{6} \mathrm{Li}$ neutron capture cross section has a $1 / \mathrm{v}$ dependence up to $50 \mathrm{keV}$, followed by a resonance peak at $220 \mathrm{keV}$, and then a further drop. This means that most neutron captures in a lithiated plastic scintillator will be at or near thermal energy, with fast neutrons undergoing one or more scattering reactions prior to capture.

As a consequence of these factors, neutron interactions in lithiated plastic scintillator will follow one of two patterns. Fast neutrons entering the scintillator will either pass through it without interacting or scatter off a hydrogen or carbon atom, depositing energy and producing a scintillation pulse with a size proportional to the energy deposition. Many neutrons that interact in the scintillator will scatter repeatedly prior to either exiting the scintillator or being captured by a ${ }^{6} \mathrm{Li}$ atom. Only fast neutrons have sufficient energy to create large scintillation pulses. The velocity of fast neutrons ensures that time delays between scatter reactions (and therefore scintillation pulses) will 
be no more than a few nanoseconds. Thus scintillation pulses from scattering reactions of an individual neutron are essentially simultaneous.

From these two interactions patterns, two scintillation patterns result. The first scintillation pattern (hereafter referred to as a "single time event") from a fast neutron is produced when a neutron scatters and then escapes the scintillator. In this case one or more scintillator bars produce simultaneous pulses. Single time events also result from neutrons that scatter in the scintillator prior to capture but do not deposit sufficient energy in an individual scintillator bar when scattering to produce a scintillation pulse that exceeds the lower level discriminator (LLD) threshold. The second pattern (hereafter referred to as a "double time event") is produced by a neutron scattering and depositing enough energy to trip the LLD before being captured by ${ }^{6} \mathrm{Li}$. In a double time event one or more scintillator bars produce simultaneous signals followed by a time delay and then another bar producing a signal.

The LLD threshold for the neutron scatter scintillation pulse is taken as $60 \mathrm{keVee}$. This corresponds to the deposition of approximately $500 \mathrm{keV}$ in the scintillator via proton recoil.

\section{4b. Neutron Detection Probability}

As described in the previous section, there are two neutron detection scintillation patterns. Figure 2 shows the neutron detection probability for both these patterns and in total for lithium concentrations of $0.1,0.5$, and $2 \%$ by weight for scintillator thicknesses of 10,20 , and $30 \mathrm{~cm}$. It is seen that for $10 \mathrm{~cm}$ thick scintillator, the total neutron detection probability peaks at slightly over 0.7 for $1.5 \mathrm{MeV}$ neutrons at all lithium concentrations. Neutron detection probability using the double time event pattern is 0.23 , 
0.28 , and 0.32 for $0.1,0.5$, and $2 \%$ lithium content. For $20 \mathrm{~cm}$ thick scintillator, the corresponding values are 0.85 total detection probability and $0.45,0.51$, and 0.54 for the three lithium concentrations using the double time event pattern. For $30 \mathrm{~cm}$ thick scintillator, these values rise to 0.87 total detection probability and $0.49,0.55$, and 0.58 for the double time event pattern. Excellent detection probabilities are achieved even at very high neutron energies; for example, the $0.5 \%$ lithium $20 \mathrm{~cm}$ thickness case gives total and double time event detection probabilities of 0.59 and 0.22 , respectively, for 10 $\mathrm{MeV}$ neutrons.

Figure 3 shows the variation in double time event, single time event, and total detection probability with lithium content for $1.5 \mathrm{MeV}$ neutrons for 10,20 , and $30 \mathrm{~cm}$ thick scintillator. Double time events are particularly significant as the detector may be operated in a capture gated mode in which the combination of both events with a short (microsecond scale) time delay separating them is taken as an indication of a neutron (as opposed to a gamma interaction). [29,30] It is seen that only very low concentrations $\left(0.1 \%\right.$ or greater) of ${ }^{6} \mathrm{Li}$ are required to achieve neutron detection efficiencies using the capture gated method that equal or exceed the present state-of-the-art for neutron detection. [31] It is also seen that neutron detection probability, especially double time event detection probability, increases rapidly with total scintillator thickness up to around $20 \mathrm{~cm}$, after which detection probability quickly levels off.

The detection probability for the plutonium spontaneous fission neutron spectrum in total and for double time events is shown in Figure 4. Again it is seen that detection probability using the double time event method nearly doubles when the scintillator thickness is increased from $10 \mathrm{~cm}$ to $20 \mathrm{~cm}$, but rises by no more than fifteen percent 
when thickness is increased from $20 \mathrm{~cm}$ to $30 \mathrm{~cm}$. Total detection probability increases by less than a third in total between the $10 \mathrm{~cm}$ and $30 \mathrm{~cm}$ cases. Increasing lithium concentration in the scintillator from $0.1 \%$ to $2 \%$ increases total detection probability by only a few percent, but increases double time event detection probability by forty percent for the $10 \mathrm{~cm}$ case and by almost twenty percent for the $30 \mathrm{~cm}$ case. In all cases, the greatest increases with lithium concentration occur at the lowest lithium concentrations;

most of the detection probability increase occurs between $0.1 \%$ and $0.5 \%$ lithium concentration, with the increase between $0.5 \%$ and $2 \%$ lithium concentration being much more modest.

\section{4c. Neutron Energy at Capture}

The ${ }^{6} \mathrm{Li}$ neutron capture cross section is, as mentioned earlier, highly dependent on neutron energy, being quite low for very high energy neutrons and increasing rapidly with decreasing neutron energy below $50 \mathrm{keV}$. As a result, most neutrons in the lithiated plastic scintillator are captured at low energies (in the thermal to low epithermal range) after losing energy by scattering off hydrogen and carbon atoms.

Figure 5 shows the energy distributions of captured neutrons for ${ }^{6} \mathrm{Li}$ concentrations of $0.1 \%, 0.5 \%$, and $2 \%$ for $10 \mathrm{~cm}$ thick scintillator. (All neutrons had an initial energy of $2 \mathrm{MeV}$.) Energies at which peak capture occurs are $0.05 \mathrm{eV}, 0.1 \mathrm{eV}$, and $1 \mathrm{eV}$, respectively. An additional, though much lower, capture peak occurs at $220 \mathrm{keV}$ in connection with the ${ }^{6} \mathrm{Li}$ cross section resonance peak at that energy. Calculations for 20 and $30 \mathrm{~cm}$ thick scintillator produced identical results.

The energy distribution of neutrons at the point of capture by ${ }^{6} \mathrm{Li}$ is, from a practical standpoint, independent of the initial energy of the neutrons over the fission 
energy range as almost all neutron capture occurs at neutron energies below those produced by fission. Neutrons from spontaneous and neutron-induced fission will almost always lose a large fraction of their energy to scattering reactions prior to capture.

\section{4d. Time Delays Between Scatter and Capture}

Figure 6 shows the neutron capture cumulative distribution function over time for ${ }^{6} \mathrm{Li}$ concentrations of $0.1 \%, 0.5 \%$, and $2 \%$ in $10 \mathrm{~cm}$ thick scintillator. A value of 0.5 (i.e. the point at which fifty percent of the neutrons have been captured) occurs at times of 13.6, 3.0, and 0.7 microseconds, respectively. A value of 0.9 is achieved at 47.0, 10.7, and 2.7 microseconds, respectively. For $20 \mathrm{~cm}$ thick scintillator, these values are 15.0, 3.2, and 0.8 microseconds for a CDF value of 0.5 and 51.1, 11.2, and 2.8 microseconds for a CDF value of 0.9 , respectively. For $30 \mathrm{~cm}$ thick scintillator, these values are 15.3, 3.3, and 0.8 microseconds for a CDF value of 0.5 and 51.7, 11.3, and 2.9 microseconds for a CDF value of 0.9 , respectively. In all three scintillator cases the shape of the CDF curves are the same.

Looking at these figures, it is seen that average time delays are very nearly inversely proportional to the lithium concentration in the scintillator. This is as one would expect as the average distance a neutron travels through the scintillator prior to capture bears the same inverse relationship with the neutron capture cross section, and therefore the lithium concentration. By contrast, time delays are only weakly dependent on the scintillator thickness and are nearly constant for thicknesses greater than $20 \mathrm{~cm}$.

\section{4e. Spatial Distribution of Events}

In addition to a separation in time between the point at which a neutron is absorbed and the time when it first scattered, there is a distance between its first scatter 
location and its point of capture. The distribution function of this distance is important due to its effect on detector performance. When the detector is run in a capture gated mode for neutron detection, the maximum count rate of the detector depends on the length of the time window between the neutron scatter and capture events. Within a large area detector, a spatial window around the neutron scatter event can be used to discriminate against events that are too far away from the scatter to be the capture of the same neutron. The use of spatial windows will allow different regions of the detector to count neutrons simultaneously, thereby increasing the maximum count rate of the device.

Figure 7 shows the distribution of distance between the first detected neutron scatter event and neutron capture location for $2 \mathrm{MeV}$ neutrons in 10,20, and $30 \mathrm{~cm}$ thick scintillator. For $10 \mathrm{~cm}$ thick scintillator, average distances are $7.9,7.6$, and $7.2 \mathrm{~cm}$ for $0.1 \%, 0.5 \%$, and $2 \%{ }^{6} \mathrm{Li}$ concentrations, respectively. These values rise to $9.8,9.3$, and $9.0 \mathrm{~cm}$, respectively, for $20 \mathrm{~cm}$ thick scintillator and $10.5,10.0$, and $9.6 \mathrm{~cm}$, respectively, for $30 \mathrm{~cm}$ thick scintillator.

\section{Results and Discussion: Gamma Detection}

\section{5a. Gamma Interaction Patterns}

Gamma rays interact in the plastic scintillator in one of two ways; via Compton scattering over the entire energy range of gammas considered (0.1-10 MeV) and via pair production at high energy, the minimum gamma energy for the latter being $1.022 \mathrm{MeV}$. As gammas travel at $30 \mathrm{~cm} / \mathrm{ns}$, multiple interactions of the same gamma may be considered instantaneous; time delays between interactions will be a few nanoseconds at the very greatest. Both interaction types may produce scintillations in more than one scintillator bar. Multiple gamma interactions normally occur as the maximum energy 
transfer in a single Compton scatter event is less than the gamma energy and pair production leads to the emission of two $511 \mathrm{keV}$ annihilation gammas by the positron. Additionally, an electron from a gamma interaction may exit the scintillator bar in which it originated and enter a neighboring bar (or several bars sequentially), producing a pulse in multiple bars.

\section{5b. Gamma Detection Methods and Energies}

As prior work has shown, delayed high energy gamma rays $(>3 \mathrm{MeV})$ from fission are a characteristic signature of SNM. [11,12] In addition to maximizing high energy gamma detection probability, the detector design must minimize interference from low energy gammas (i.e. it must be able to reject low energy gammas without undue loss of the high energy gamma signal). Prior work has shown that interference from gammas emitted between neutron pulse phases by non-SNM materials is low at gamma energies of $2.5 \mathrm{MeV}$ and above. [12] Therefore the plastic scintillator configuration and signal readout and processing methods chosen should maximize gamma detection probability above $3 \mathrm{MeV}$ while minimizing gamma detection probability below $2.5 \mathrm{MeV}$. As discussed earlier, the light collection efficiency for events in the scintillator bars varies with location in the bar, energy resolution will be poor. This will only affect detector performance, however, to the extent that gammas under $2.5 \mathrm{MeV}$ interfere with the signal from gammas above $3 \mathrm{MeV}$.

Gamma ray pulses for detection may be generated in a number of ways, two of which are most promising, the single interaction method and the multiple interaction method. The single interaction method is to monitor individual scintillator bars and either count based on the pulse from a single bar or the sum of coincident pulses from 
bars that are immediate neighbors to each other exceeding some LLD threshold. This in effect uses the pulse produced by a single interaction in the scintillator by a gamma. (Multiple interactions by gammas may occur in neighboring bars, this being most frequent for low energy gammas with short mean free paths in the scintillator. Such multiple interactions only improve the gamma detection probability and are in fact included in the data in the next section when they occur in the same bar.) The multiple interaction method is to sum all coincident pulses from the scintillator bars either throughout the entire device or within a large window of scintillator volume and then apply to an LLD to the aggregate pulse. This second method differs from the first in that by summing scintillation pulses from a much larger volume of detector, pulses from multiple gamma interactions (including annihilation gammas from pair production) are summed, not just pulses from a single interaction. The choice of the signal readout and processing method becomes a question of how to maximize high energy gamma detection probability to achieve high SNM sensitivity while keeping the system simple and affordable.

\section{5c. Gamma Detection Probability}

Gamma ray detection probability varies with gamma energy, scintillator thickness and size, LLD value, and choice of signal readout and processing method (analysis of single gamma interactions or total signal amplitude from multiple interactions).

Figure 8 shows the gamma detection probability as a function of energy for the single interaction method using a variety of LLDs and scintillator thicknesses of 10, 20, 30, and $40 \mathrm{~cm}$. Figure 9 shows equivalent data for the multiple interaction method (giving a total pulse amplitude) using the same LLDs and scintillator thicknesses. It is 
seen that over the entire range of scintillator thicknesses (that is, up to $40 \mathrm{~cm}$ ), high energy gamma detection probability increases substantially with each increase in thickness.

Figure 10 shows gamma detection probability as a function of energy for scintillator thicknesses of $10,20,30$, and $40 \mathrm{~cm}$ using the single interaction method and fixed LLDs of 2, 2.5, and $3 \mathrm{MeVee}$. Figure 11 shows the equivalent data for the multiple interaction (total pulse amplitude) method.

It is seen in Figures 10 and 11 that detection probabilities of energetic gammas rise substantially with increasing scintillator thickness over the range $0-40 \mathrm{~cm}$. In most cases, adding another 10 or $20 \mathrm{~cm}$ of scintillator thickness is expected to produce useful detection probability gains. It should be noted however that in practice the probability of a gamma producing a particular light emission event will be higher than indicated by Figures 10 and 11 and thus the potential for improving detection probability is lower than the data seem to indicate. The reason for this is that the values in Figures 10 and 11 are for gammas that are normally incident on the scintillator. In a real system, many would impinge on the scintillator at different angles, giving a much greater distance they must traverse before exiting the scintillator than the actual scintillator thickness. Total system cost and to a lesser extent physical availability of space in a fielded system are also important considerations in determining what scintillator thickness to use.

In addition to type of signal readout and processing method, the best LLD value to use in a system for SNM detection via high energy gammas is also heavily dependent on two other factors. These are the energy resolution of the wavelength-shifting readout fiber system (i.e. how much resolution is lost due to variability in photon detection 
efficiency) and the number and energy distribution of gammas present below the energies used for SNM detection that may interfere with the high energy gamma signal due to limitations in energy resolution.

Referring to Figure 10, it may be seen that the use of single interaction pulses yields the following results. For an LLD value of $2 \mathrm{MeVee}$, detection probability is very low or zero below a gamma energy of $2.25 \mathrm{MeV}$ and rises rapidly after this, reaching $0.15,0.27,0.35$, and 0.40 at $3 \mathrm{MeV}$ for scintillator thickness of $10,20,30$, and $40 \mathrm{~cm}$, respectively. At $5 \mathrm{MeV}$, these values rise to $0.20,0.36,0.48$, and 0.58 , respectively. For an LLD value of $2.5 \mathrm{MeVee}$, detection efficiencies are $0.08,0.14,0.18$, and 0.21 at 3 $\mathrm{MeV}$, respectively, and $0.18,0.33,0.44$, and 0.53 at $5 \mathrm{MeV}$, respectively. Finally, at an LLD of $3 \mathrm{MeVee}$, detection efficiencies are $0.07,0.12,0.16$, and 0.18 at $3.5 \mathrm{MeV}$, respectively, and $0.16,0.29,0.39$, and 0.46 at $5 \mathrm{MeV}$, respectively.

A comparison of Figures 10 and 11 reveals that the difference between the high energy gamma detection probabilities for the single interaction and multiple interaction methods is generally modest, especially at the higher gamma energies. As the single interaction method is simpler and gives much less opportunity for accidental summing of pulses from separate, coincident gammas, it seems the best choice assuming reasonable pulse amplitude resolution from the light collection system. At the same time, it should be noted that for gamma energies in $\mathrm{MeV}$ that are numerically very near to the LLD value in MeVee, the difference in detection probability between the two methods can exceed a factor of two and care should thus be exercised. An LLD of between 2.5 and 3 MeVee seems best. In general, the LLD value should be kept as low as is still consistent 
with minimizing interference from non-SNM gammas as high energy gamma detection probability falls as the LLD is raised.

Another possible method of detecting high energy gammas is to selectively accept events based on pulses appearing simultaneously in different scintillator bars and each pulse exceeding an LLD value, with potentially different LLD values being used for the largest and next largest pulses. This method was considered but found to be substantially inferior to both the single and multiple interaction methods.

\section{Prospects for Neutron-Gamma Discrimination}

\section{6a. Significance of Neutron-Gamma Discrimination}

Practically all neutron detectors are sensitive to gamma rays to one extent or another, and distinguishing between neutrons and gammas is normally an issue of great importance and interest. In an active neutron interrogation system that uses both neutron and high energy gamma detection for SNM identification with the number of neutrons and high energy gammas detected being proportional to the quantity of SNM present, it is only strictly necessary to distinguish between neutrons and lower energy gammas, although separation of the neutron and high energy gamma signals may provide useful information such as the likely identity of the SNM material.

\section{6b. Pulse Height and Shape Discrimination}

The preferred method for neutron-gamma discrimination is pulse height discrimination, this being effective if most neutron pulses produced by a detector are of greater amplitude than most gamma pulses. An alternate method is pulse shape discrimination, which is effective if neutron pulses have a measurably different shape 
than gamma pulses, the difference normally being in the time constant of the pulse tail decay.

Neither the pulses from the proton recoil from neutron scattering reactions in plastic scintillator generally nor the ${ }^{6} \mathrm{Li}$ neutron capture reaction in the lithiated plastic scintillator are large enough to reliably distinguish from energetic gammas depositing approximately $0.5 \mathrm{MeV}$ in an interaction in the scintillator. It will be practical to rule out some fraction of the gamma events as not being neutron capture events due to being either too large or too small and this will substantially assist in neutron-gamma discrimination but by itself will be insufficient.

Pulse shape discrimination has been used successfully with organic liquid scintillator (generally quite similar to plastic scintillator in its chemistry and response characteristics) and is now being applied to plastics. [32-34] However, light collection using the scintillator bar/WLS fiber readout configuration is unlikely to work with pulse shape discrimination due to the low number of photons from an event actually reaching the MAPMT compared with the number of photons initially emitted in a scintillation event and the change in pulse shape from the readout fibers (the fibers re-emit shifted photons over a period of nanoseconds following initial photon absorption).

\section{6c. Neutron Identification by Interaction Pattern}

Another method of neutron-gamma discrimination, and one that is much more suited to this detector concept, is to operate the detector in a neutron capture gated mode as discussed earlier. In this mode, a time counter is started when a fast neutron scatters in the scintillator. A second event occurring within a brief time window (microseconds) 
after the first event and consistent in amplitude with a neutron capture event (given limitations in energy resolution) is taken as such an event.

In capture gating, false neutron counts from gammas can occur in several different ways. In the first, a gamma interaction is taken as a neutron scattering event (there being no way to distinguish these two) and used as a start pulse for the capture time window. A second gamma can then produce a pulse and trigger a count. This is the most serious case as no neutron contributed to the count. Gammas may also be confused for neutron scattering events and thus be used as a start pulse or for neutron capture events and thus trigger a count, with either a neutron scatter or a capture causing the other event (start or trigger). These latter possibilities are not as problematic as a real neutron does result in a neutron pulse, but they change the relationship between the number of neutrons entering the detector and the number of neutron counts registered and thus the detector reading.

Capture gating is an effective way of distinguishing between neutrons and gammas under two conditions. The first is that the average time between gamma events is large compared to the duration of the capture window; otherwise the false neutron count rate from gammas will be unacceptably high. (The higher the lithium content of the scintillator, the shorter the capture window can be and the higher the gamma flux that can be tolerated.) The second is that substantial numbers of fast neutrons are present in the neutron field as neutrons must deposit at least $500 \mathrm{keV}$ of energy to meet the LLD of 60 keVee scintillation light emission intensity for triggering a start pulse.

The actual ratio of true neutron counts to false neutron counts will depend on the relative and absolute numbers of neutrons and gammas interacting in the detector, the 
energy resolution of the detector (e.g. its ability to minimize false neutron capture events from gammas by rejecting events that are too large or small to be ${ }^{6} \mathrm{Li}$ capture reactions), the lithium content of the plastic scintillator (the higher the lithium content, the shorter the capture gated window and thus the lower the potential for gamma interference), and the detector settings (e.g. energy and time windows). True and false neutron count rates and their ratio can be calculated for different applications if these values are known.

\section{6d. Using Detector Geometry to Improve Neutron-Gamma Discrimination}

Detector geometry considerations can be used to minimize false counts from gamma rays. For example, if lithiated plastic scintillator bars are interspersed with plain plastic scintillator bars as discussed earlier, events within the capture time window that occur in plain plastic scintillator bars are therefore known not to result from neutron capture events. As another example, neutron capture events tend to usually occur near the location at which the neutron first scattered as shown in Figure 7. This means that many gamma events can be determined not to be neutron captures by virtue of occurring in a scintillator bar that is some distance from the bar that started the capture gate window.

\section{Sensitivity to SNM}

\section{7a. Measures of Sensitivity}

A key performance metric for any neutron or gamma detector for security applications is its sensitivity for the detection of nuclear material. For a particular detector, the quantity of interest is usually one of the maximum stand-off detection distance, the counting time required, and the detectable source quantity, with one of those quantities varying and the others being held constant. Although the following sections 
deal with the maximum stand-off distances for the detection of fixed and moving sources, the basic equations may be easily rearranged to solve for other quantities given a fixed stand-off distance.

7b. Derivation of Stand-Off Distance for a Fixed Source

For a point $P$ located a distance $z$ from the center of a rectangular area $W \times L$ on a line perpendicular to the center of that area, the solid angle $\Omega$ subtended by the rectangular area is given by

$\Omega=4 \tan ^{-1}\left(\frac{\varepsilon}{\eta \sqrt{1+\varepsilon^{2}+\eta^{2}}}\right)$,

where $\varepsilon \equiv W / L$ and $\eta \equiv 2 z / L$. This is illustrated in Figure 12. For a rectangular detector directly facing a source, $z$ is the stand-off distance and $W$ and $L$ are the width and length, respectively, of the detector.

The term inside the $\tan ^{-1}$ operation in Eq. (1) may be rewritten as

$\frac{W / L}{\left(\frac{2 z}{L}\right) \sqrt{1+\frac{W^{2}}{L^{2}}+\frac{4 z^{2}}{L^{2}}}}$.

If $z$ is much larger than both $W$ and $L$, it is trivial to show that the divisor of Eq. (2) approximates to

$\frac{4 z^{2}}{L^{2}}$.

Substituting this back into Eq. (2) yields the following:

$\frac{W L}{4 z^{2}}$.

Since $W L$ is simply the area of the detector, we can rewrite Eq. (1) as 
$\Omega=4 \tan ^{-1}\left(\frac{A}{4 z^{2}}\right)$

The relationship between the number of neutrons emitted by the source and the number of neutrons entering the detector may be expressed as

$\frac{S \Omega}{4 \pi}=A F_{s}$,

where $S$ is the source strength in neutrons per second, $A$ is the area of the detector, and $F_{S}$ is the neutron flux at the detector due to the source. Note that the division of $\Omega$ by $4 \pi$ transforms the solid angle the detector represents to the source into a fraction of the total solid angle. Eq. (6) may be rearranged to give

$\Omega=\frac{4 \pi A F_{s}}{S}$

Eqs. (5) and (7) may be combined and $z$ may be solved for. However, $F_{s}$ must be known in order to find a numeric value for $z$; therefore we will derive an expression for $F_{s}$ prior to solving for $z$.

Seymour et al have previously derived the equation for $n_{\sigma}$, the confidence (expressed in sigma) that an increase in detector count rate has correctly signaled the detection of a source, as

$n_{\sigma}=F_{s} \sqrt{\frac{\varepsilon A t}{F_{B}}}$,

where $F_{S}$ is the flux due to the source, $\varepsilon$ is the probability of detecting a neutron entering the detector, $A$ is the detector area, $t$ is the measurement time, and $F_{B}$ is the background neutron flux. [31] Eq. (8) may be easily rearranged into the form

$F_{s}=n_{\sigma} \sqrt{\frac{F_{B}}{\varepsilon A t}}$ 
to yield the source flux at the detector required to detect the source given specified values of detection confidence, background flux, neutron detection probability, detector area, and counting time.

Combining Eqs. (5) and (7) produces the equality

$$
\tan ^{-1}\left(\frac{A}{4 z^{2}}\right)=\frac{\pi A F_{s}}{S},
$$

after minor cancellation. Rearranging and solving for the stand-off distance $z$ gives

$$
z=\sqrt{\frac{A}{4 \tan \left(\frac{\pi A F_{s}}{S}\right)}},
$$

where all terms have been previously defined and the value of $F_{s}$ is determined using Eq. (9). Care should be taken to use consistent units when calculating the stand-off distance as some values (e.g. neutron flux and detector area) are often typically expressed using different units (e.g. $\mathrm{cm}^{2}$ and $\mathrm{m}^{2}$ ).

In addition to detecting a source that has a fixed location relative to a detector, there is also great interest in detecting neutron sources that are in motion relative to a detector; for example, a source in a truck or train passing a fixed detector located along an artery of transportation. Deriving an expression for the maximum stand-off distance is far more complicated for this case than for a fixed source case. Accordingly, a significant approximation will be used to derive an expression for the stand-off distance given source-detector motion.

\section{7c. Derivation of Stand-off Distance for a Source in Motion}

Assume a neutron detector operates with a fixed set of detection parameters (e.g. $n_{\sigma}, A$ ). If the neutron detector directly faces a neutron source and a count time $t$ is used, 
the maximum stand-off distance $z$ may be found using Eq. (11). If the neutron source is in motion, it will still be detectable within time $t$ provided two conditions are met. These are:

(i) The source stays within a distance $z$ of the detector during $t$.

(ii) The source remains in an area such that the solid angle the detector represents to the source is not significantly diminished; in other words, the source remains approximately in front of the detector.

An example of this is shown in Figure 13 in which a source moves a distance $l$ along a chord of a circle formed by the detector as its center and the stand-off distance $z$ as its radius, the chord being parallel to the detector plane. In this example, the angle $\theta$ is sufficiently small that the second condition given above is met. The distance $d$ is then the estimated maximum stand-off distance for a source in motion past the detector along the chord, with the value of $l$ being the distance the source travels at speed $v$ during time $t$. Referring again to Figure 13, the relationship between $d, z$, and $l$ is seen to be $d=\sqrt{z^{2}-0.25 l^{2}}$

Using Eqs. (9), (11), and (12), it is trivial to show that the maximum stand-off distance for the source in motion case occurs for a value of $t$ when

$$
\frac{\partial}{\partial t}\left(A\left[\tan \left(\frac{\pi n_{\sigma} \sqrt{A F_{B}}}{S \sqrt{\varepsilon t}}\right)\right]^{-1}-v^{2} t^{2}\right)^{0.5}=0
$$

Once the optimum value of $t$ is known, the maximum stand-off distance for the source in motion case is given by 
$d=0.5\left(A\left[\tan \left(\frac{\pi n_{\sigma} \sqrt{A F_{B}}}{S \sqrt{\varepsilon t}}\right)\right]^{-1}-v^{2} t^{2}\right)^{0.5}$

In practice however, $t$ is not normally a continuous variable as counting times are of either fixed duration or are a multiple of a fixed time interval. To find $d$, therefore, discrete values of $t$ corresponding the actual counting intervals used by the detector should be used and the lowest value of $d$ (or the value of $d$, if only one counting interval is used) taken as the maximum stand-off distance when the source is in motion.

\section{7d. Assumptions}

Several important assumptions are made during the preceding derivations. These are:

(i) The travel path of the source is assumed to be parallel to the detector plane. This will be true for sources with constrained paths running in front of a detector bank (for example, a source inside a train passing a monitoring system in a rail yard), but will often be the case in many applications.

(ii) No provision is made for intervening shielding. Shielding of the source may be accounted for by reducing the value of $S$ by some fraction, but accounting for shielding by intervening air requires a reduction in the number of neutrons reaching the detector that varies with $z$ and $d$. Shielding by air is only significant, however, for very large source to detector distances (e.g. hundreds of meters).

(iii) Other environmental effects on neutron economy (e.g. scattering off the ground or nearby buildings) are not considered. This is a conservative assumption. 


\section{7e. Stand-Off Distance}

The stand-off distance will vary substantially with the source size and type, interrogation method (passive or active), detector operation mode (e.g. neutron only or neutron/gamma detection, double time events only for neutrons or all events counted), detector geometry and design, and so forth.

Passive detection of SNM based on neutron emission is of great interest for use in security applications. To calculate a realistic stand-off distance for an SNM detector suing passive neutron detection, the following constant parameters were used for all cases:

(i) The source was assumed to be a nuclear warhead primary containing $5 \mathrm{~kg}$ of plutonium and having a $\mathrm{k}_{\text {eff }}$ (multiplication constant) of 0.9 , thus yielding a total of $2.96 \times 10^{6}$ neutrons $/ \mathrm{sec}$.

(ii) Neutron detection probability was taken as 0.3 based on the double time event neutron detection probability for $20 \mathrm{~cm}$ thick scintillator with $0.5 \%$ lithium concentration with twenty percent of the counts rejected due to falling outside the capture time window, being captured at too great a distance from the scatter location, and other loss mechanisms. This is a conservative value.

(iii) A value of 5.2 was used for $n_{\sigma}$, the confidence that an increase in detector count rate has correctly signaled the detection of a source.

(iv) A background neutron flux of 0.007 neutrons $/ \mathrm{cm}^{2} . \mathrm{sec}$ was used.

(v) Counting times were normally whole multiples of 5 seconds, except in specifically noted special cases.

Three variable parameters were used. They were: 
(i) Detector area. Sizes of $10 \mathrm{~m}^{2}, 1 \mathrm{~m}^{2}$, and $0.1 \mathrm{~m}^{2}$ were used. These correspond to a $5 \mathrm{~m} \mathrm{x} 2 \mathrm{~m}$ very large area scanner for maximum sensitivity, a $1 \mathrm{~m}^{2}$ scanner, and a $0.1 \mathrm{~m}^{2}$ portable device.

(ii) Shielding. Probabilities of neutrons emitted towards the detector reaching the detector of $1,0.5$, and 0.1 were used, corresponding to no shielding, moderate shielding, and heavy shielding. (These descriptions are subjective.)

(iii) Source motion. Fixed, $8 \mathrm{kph}(5 \mathrm{mph})$ speed, and $72 \mathrm{kph}(45 \mathrm{mph})$ speed cases were used. In all cases the maximum count time was fixed at $60 \mathrm{sec}$.

Table 1 lists the stand-off distance for the stationary source case using a one minute counting time. A $10 \mathrm{~m}^{2}$ very large area detector can detect a nuclear warhead primary at a range of $269 \mathrm{~m}$ if no intervening shielding is present. If nine-tenths of the emitted neutrons are shielded, the detection range drops to $85 \mathrm{~m}$. The stand-off distance varies according to the square root of the source strength and the quadruple root of the detector size. Thus the $0.1 \mathrm{~m}^{2}$ detector has a stand-off distance of $27 \mathrm{~m}$ for the heavy shielding case.

The stand-off distances and corresponding counting times for the $8 \mathrm{kph}$ source motion case are listed in Table 2. Due to geometrical effects, the reduction in stand-off distance is least pronounced for very large stand-off distances and most pronounced for short stand-off distances. Thus the $10 \mathrm{~m}^{2}$ detector has a stand-off distance of $261 \mathrm{~m}$ for an unshielded source, almost the same as the stationary case, whereas the stand-off distance for a heavily shielded source using the $0.1 \mathrm{~m}^{2}$ detector is $13 \mathrm{~m}$, less than half that of the stationary case. This is in part due to the counting times necessarily decreasing as 
the source spends less time inside the zone around the detector (see Figure 13) as the radius of the zone around the detector (the stand-off distance) is reduced.

Table 3 lists the stand-off distances and corresponding counting times for the 72 $\mathrm{kph}$ source motion case. In the best case, a $10 \mathrm{~m}^{2}$ device can detect a warhead at $140 \mathrm{~m}$ using a $10 \mathrm{sec}$ counting interval. Only data for the $10 \mathrm{~m}^{2}$ and $1 \mathrm{~m}^{2}$ detectors and the no shielding and moderate shielding cases are shown as the other cases either require counting intervals under $5 \mathrm{sec}$ or may not be feasible. It should be noted that for the case of the $1 \mathrm{~m}^{2}$ detector and moderately shielded source, the stand-off distance can be increased from $28 \mathrm{~m}$ to $41 \mathrm{~m}$ by using a counting time of $2 \mathrm{sec}$ instead of $5 \mathrm{sec}$. The one downside to shifting to counting times that are whole multiples of, for example, $2 \mathrm{sec}$ instead of $5 \mathrm{sec}$ is a modest increase in the rate of false positives due to statistical fluctuations in neutron background counting.

\section{Comparison with Existing Technologies}

\section{8a. SNM Sensitivity}

Sensitivity to SNM is governed by a host of factors; for example, the size of a detector, the ambient background neutron flux, the confidence level used as an indicator of the presence of SNM, the extent to which the SNM is shielded, and so forth. Despite the multitude of factors affecting sensitivity, the performance of any given detector depends primarily on two factors; its intrinsic detection probability (efficiency) and its false count rate from sources such as radiation particles it is not intended to detect and electronic noise.

Active interrogation is complex and calculating very specific values for SNM sensitivity using active methods is beyond the scope of this work. The standard passive 
SNM detection method is to use the neutrons emitted by plutonium and other transuranics. The present state-of-the-art technologies are ${ }^{3} \mathrm{He}$ and lithiated glass fiber systems. [31] These have Pu spontaneous fission neutron detection probabilities of 0.15 (typical) and 0.18 , respectively. This compares with 0.30 for a system using $20 \mathrm{~cm}$ thick plastic scintillator with a lithium concentration of $0.5 \%$. Figure 14 illustrates this.

The most important cause of the large improvement in neutron detection probability that lithiated plastic scintillator represents over lithiated glass and ${ }^{3} \mathrm{He}$ tubes is the mixing of the neutron target material $\left({ }^{6} \mathrm{Li}\right)$ with the moderator. The greater the extent to which the neutron target material is confined within specific zones of a detector, particularly when the neutron moderating properties of those zones are poor, the greater the likelihood of an initially fast neutron exiting the detector with passing through the target zones while at a thermal or near thermal energy. For example, in a system consisting of ${ }^{3} \mathrm{He}$ tubes sandwiched between layers of polyethylene moderator, many neutrons are simply backscattered by the front layer of polyethylene and never reach the ${ }^{3} \mathrm{He}$ tubes, thus ensuring that they cannot be detected. Conversely, the greater the homogeneity of a detector (i.e. the extent to which the neutron target material is dispersed throughout the moderator), the higher the fast neutron detection probability.

In contrast to the neutron detection probability, which is for all intents and purposes constant for a particular energy spectrum regardless of flux, the false count probability from gamma rays (gammas mistaken for neutrons) will vary, as explained in Section $6 c$, with the characteristics of both the incident gamma field (e.g. flux) and the neutron field. It is beyond the scope of this work to examine this in detail. Methods for 
minimizing gamma interference with the neutron signal (e.g. use of double time events only, spatial and time neutron capture windows) are described elsewhere in this work.

\section{8b. Limiting Factors Affecting Sensitivity}

The most important limiting factor in the lithiated plastic scintillator is the lithium content that can be achieved while retaining sufficient scintillation emission intensity and optical transparency. There are two major reasons for this.

Although it is true that when substantial thicknesses $(10-20 \mathrm{~cm})$ of lithiated plastic scintillator are used neutron detection probability is relatively insensitive to lithium content above $1 \mathrm{wt} \%$ or so, the scintillator thickness required to achieve a given neutron detection probability using capture gating will fall approximately inversely with lithium concentration, provided this takes the form of reducing the thickness of lithiated plastic scintillator bars and adding additional plain plastic scintillator bars between them. This is important because the smaller the volume of lithiated plastic scintillator for detecting neutron captures, the lower the potential for interference from gamma rays.

Second, the greater the lithium concentration, the shorter the delay between the

first scatter of a fast neutron and its capture by ${ }^{6} \mathrm{Li}$ and thus the shorter the capture gate time window needs to be. Decreasing the window duration reduces the false neutron count rate from gammas and increases the maximum system counting rate.

\section{Recommendations for Future Work}

The scintillation light collection efficiency of the wavelength-shifting (WLS) fiber readout system and the resulting energy resolution of pulses will affect detector performance for both neutron and high energy gamma detection, especially the latter. For example, the lower the resolution, the higher the LLD setting needed to discriminate 
between high energy gammas and lower energy ones. The scintillation light collection system should be studied with the goal of determining how best to maximize light collection efficiency while keeping cost and complexity as low as possible.

Neutron-gamma discrimination is a key performance metric of the lithiated plastic scintillator. A number of methods for discriminating between neutrons and gammas are described in this paper, such as the use of neutron capture time and spatial windows following a detectable neutron scatter event, the use of only double time events for neutron detection, and pulse height discrimination to the extent that the energy resolution of the fiber readout system allows to reject some fraction of the gamma events as definitely not being due to neutrons. Neutron-gamma discrimination should be studied more extensively to determine the best approach to accomplishing it and how effective that approach will be.

The methods for calculating stand-off distance, particularly for the source in motion case, use several assumptions. It would be worthwhile to improve on the standoff distance calculation methods to account more comprehensively for shielding and geometric effects.

An optimized detector design could be created that takes into account all the factors touched on in this paper along with cost considerations such as minimizing the amount of lithiated plastic scintillator required by interspersing lithiated and plain plastic scintillator bars. Such a design would also improve neutron-gamma discrimination by enabling all events in plain plastic scintillator bars to be eliminated as possible ${ }^{6} \mathrm{Li}$ neutron capture events. Such an optimization would require that an appropriate 
price/performance tradeoff for a full system be known. A complete system should be constructed and tested to verify its performance.

\section{Conclusions}

The use of extruded plastic scintillator bars in conjunction with a wavelengthshifting (WLS) fiber readout system provides a simple and inexpensive method for constructing very large volume neutron and high energy $(>3 \mathrm{MeV})$ gamma detectors. Such detectors can sensitively detect SNM using both active and passive interrogation methods.

Fast neutrons produce two distinct pulse patterns in the detector, the double time event pattern giving the best neutron-gamma discrimination. In the double time event case a pulse from a neutron scattering off a hydrogen atom is followed within a brief time period (microseconds) by a pulse from the neutron being captured by a ${ }^{6} \mathrm{Li}$ atom. Time and spatial windows for neutron capture around the scatter location may be used to enhance neutron-gamma discrimination. The use of lithiated plastic scintillator $20 \mathrm{~cm}$ thick with a $0.5 \%$ lithium concentration is sufficient to give a plutonium spontaneous fission neutron detection probability of 0.3 after allowances for neutron events lost in the course of rejecting gamma events. Increasing the scintillator thickness beyond $20 \mathrm{~cm}$ produces only small improvements in neutron detection probability.

Detection probabilities around 0.5 may be achieved using $40 \mathrm{~cm}$ thick scintillator for high energy gammas (>3 MeV) using LLD values corresponding to 2-2.5 MeVee of scintillation light emission. The mean free path of high energy gammas in the scintillator is substantially larger than that of fast neutrons and high energy gamma detection probability can be further increased using scintillator thicknesses greater than $40 \mathrm{~cm}$. 
Neutron-gamma discrimination can most effectively be accomplished by analyzing interaction patterns to identify neutron double time event occurrences and by applying time and spatial windows to eliminate many gamma events from consideration. The light collection efficiency of the WLS fiber readout system will likely preclude the use of pulse shape discrimination. Pulse height analysis (PHA) will enable rejection of some gamma events but by itself will not yield sufficient neutron-gamma discrimination for a practical system, thus the use of interaction pattern analysis with conjunction with PHA is necessary. Neutron-gamma discrimination can be further improved by mixing plain and lithiated plastic scintillator bars in a configuration with optimized neutronics, as pulses from plain plastic scintillator bars are known not to be neutron capture events by ${ }^{6} \mathrm{Li}$. Investigation into the use of both plain and lithiated plastic scintillator bars in a detector is recommended for future investigation.

Equations for calculating stand-off detection distance were derived for both stationary and moving source cases. In the case of passive detection, a very large area $\left(10 \mathrm{~m}^{2}\right)$ detector can, in a completely unshielded case, detect a stationary nuclear warhead primary containing $5 \mathrm{~kg}$ of plutonium at a distance of $269 \mathrm{~m}$ using a one minute count. For the same source passing the detector at speeds of $8 \mathrm{kph}$ and $72 \mathrm{kph}$, the stand-off detection distances are $261 \mathrm{~m}$ and $140 \mathrm{~m}$, respectively.

Systems based on lithiated plastic scintillator will be approximately twice as sensitive as the existing state-of-the-art for fast neutron detection using large area detectors when using the double time event neutron detection method.

Recommendations for future work include optimizing the WLS fiber readout system, further developing neutron-gamma discrimination methods and quantifying their 
performance, improving the methods for estimating stand-off detection distance, and designing, developing, and testing an optimized SNM detection system.

\section{Acknowledgements}

ACS and SAW were supported by a Small Business Innovative Research (SBIR)

Grant from the US Department of Energy (DOE) under Contract DE-FG02-01ER83224.

Funding of this work by the DOE under the SBIR grant does not constitute endorsement

by the DOE. HJI and SD were supported by an NA-22 task from the DOE Office of

Nuclear Nonproliferation and a CRADA funded from the SBIR. ORNL is managed by

UT-Battelle for the DOE under contract DE-AC05-00OR22725.

\section{References}

[1] D. Reilly, N. Ensslin, H. Smith, S. Kreiner, "Passive Nondestructive Assay of Nuclear Materials," NUREG/CR-5550 (1991), available at www.ntis.gov.

[2] A. E. Evans, M. M. Thorpe, J. J. Malanify, Transactions of the American Nuclear Society 15, 673 (1972).

[3] S. T. Hsue, Atomic Energy Review 16, 89 (1978).

[4] H. O. Menlove, T. W. Crane, Nuclear Instruments and Methods 152, 549 (1978).

[5] P. Campbell, D. G. Boase, E. M. Gardy, Analytical Chemistry in Nuclear Fuel Reprocessing 296 (1978).

[6] A. Lyoussi, et al, Nuclear Instruments and Methods B 160, 280 (1999).

[7] F. Jallu, et al, Nuclear Instruments and Methods B 170, 489 (2000).

[8] A. C. Stephan, et al, Transactions of the American Nuclear Society 85, 440 (2001).

[9] McElroy, et al, "Design and performance of the Integrated Waste Assay System (IWAS)," Proceedings of the INMM 44 ${ }^{\text {th }}$ Annual Meeting (2003).

[10] G. L. Molnar, Zs. Revay, T. Belgya, Nuclear Instruments and Methods B 213, 389 (2004). 
[11] H. O. Menlove, R. A. Forster, J. L. Parker, D. B. Smith, Nuclear Technology 20, 124 (1973).

[12] D. Slaughter, et al, "Detection of special nuclear material in cargo containers using neutron interrogation," UCRL-ID-155315 (2003), available at www.ntis.gov.

[13] L. R. Greenwood, N. R. Chellew, Nuclear Instruments and Methods 165 (1979) 129131.

[14] V. R. Negina, V. N. Popov, V. V. Nazarov, Instruments and Experimental Techniques 23(5), 1118-1120 (1980), translated from Pribory i Tekhnika Eksperimenta 23(5), 60-62 (1980).

[15] S. Ait-Boubker, et al, Nuclear Instruments and Methods A 277, 461-466 (1989).

[16] H.-J. Im, S. Saengkerdsub, A. C. Stephan, M. D. Pawel, D. V. Holcomb, S. Dai, "Transparent solid-state lithiated scintillators based on self-assembly of poly(styreneb-ethylene oxide) block co-polymer architectures," accepted for publication in Advanced Materials.

[17] G. E. Ham, Ed., Vinyl Polymerization, Marcel Dekker, New York, 1967.

[18] D. F. Anderson, et al, Proceedings of the 2000 IEEE Nuclear Science Symposium 1, $141(2000)$.

[19] A. Pla-Dalmau, A. D. Bross, V. V. Ryalkin, Proceedings of the 2003 IEEE Nuclear Science Symposium 1, 102 (2004).

[20] Itasca Plastics, St. Charles, IL, www.itascaplastics.com.

[21] J. Oliver, et al, Proceedings of the 2003 IEEE Nuclear Science Symposium 1, 206 (2004).

[22] The MINOS Collaboration, The MINOS Technical Design Report, Chapters 5 and 6, available at www-numi.fnal.gov:8875/minwork/info/tdr/minos_tdr.html.

[23] Courtesy of Dr. Anna Pla-Dalmau, Fermi National Accelerator Laboratory, Batavia, IL, www.fnal.gov.

[24] J. F. Briemeister (Ed.), "MCNP - a general Monte Carlo n-particle transport code, version 4C2," LA-12625-M, Los Alamos National Laboratory (2001).

[25] S. A. Pozzi, E. Padovani, M. Marseguerra, Nuclear Instruments and Methods A 513, 550 (2003). 
[26] J. T. Mihalczo, J. A. Mullens, J. K. Mattingly, T. E. Valentine, Nuclear Instruments and Methods A 450, 531 (1998).

[27] S. A. Pozzi, J. A. Mullens, J. T. Mihalczo, Nuclear Instruments and Methods A 524, 92 (2004).

[28] S. E. Jones, et al, Proceedings of the $10^{\text {th }}$ International Conference on Cold Fusion (2003). Available at www.lenr-canr.org/acrobat/JonesSEchargedpar.pdf.

[29] W. C. Feldman, G. F. Auchampaugh, R. C. Byrd, Nuclear Instruments and Methods A 306, 350 (1991).

[30] G. F. Knoll, Radiation Detection and Measurement, $3^{\text {rd }}$ ed., John Wiley \& Sons, New York, 1999.

[31] R. S. Seymour, et al, Proceedings of the SPIE 3536, 148 (1998).

[32] R. E. Howe, Nuclear Instruments and Methods in Physics Research 190, 309 (1981).

[33] J. Neal, et al, "Pulse shape discrimination for the Nuclear Materials Identification System (NMIS)," Proceedings of the $44^{\text {th }}$ INMM Annual Meeting (2003).

[34] S. Normand, B. Mouanda, S. Haan, M. Louvel, Nuclear Instruments and Methods A 484, 342 (2002). 
Figures and Illustrations

Figure 1.

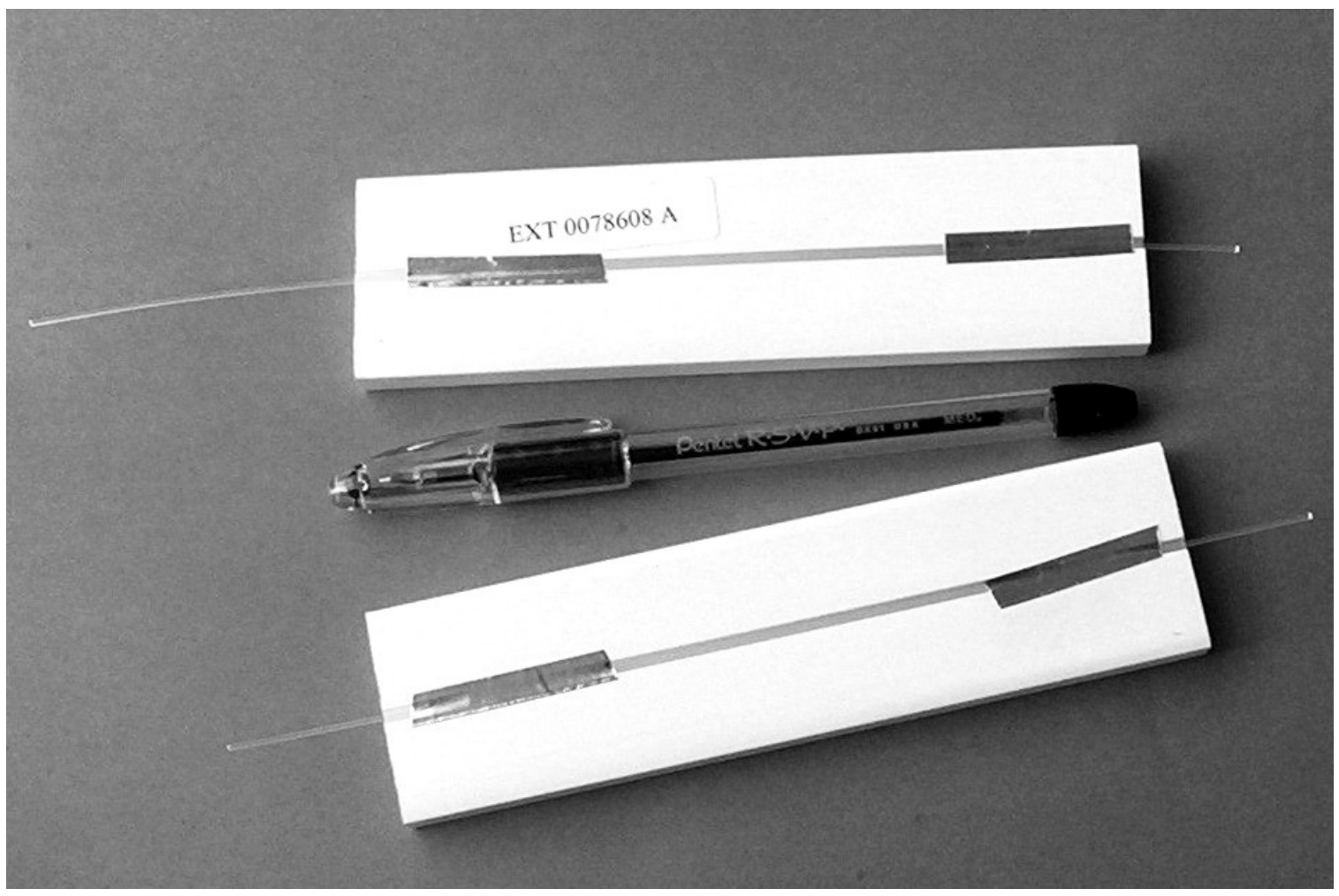


Figure 2.

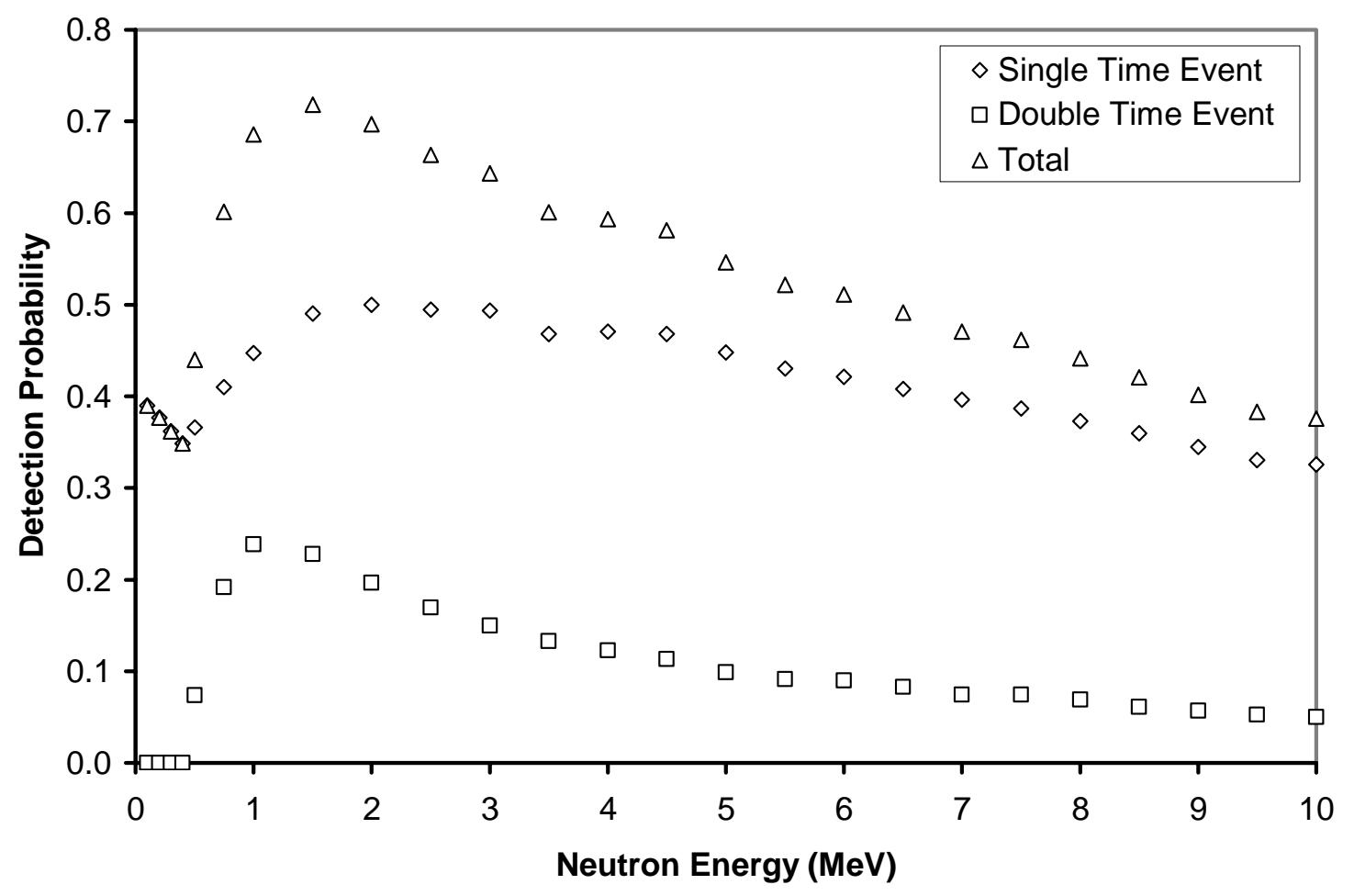

(a)

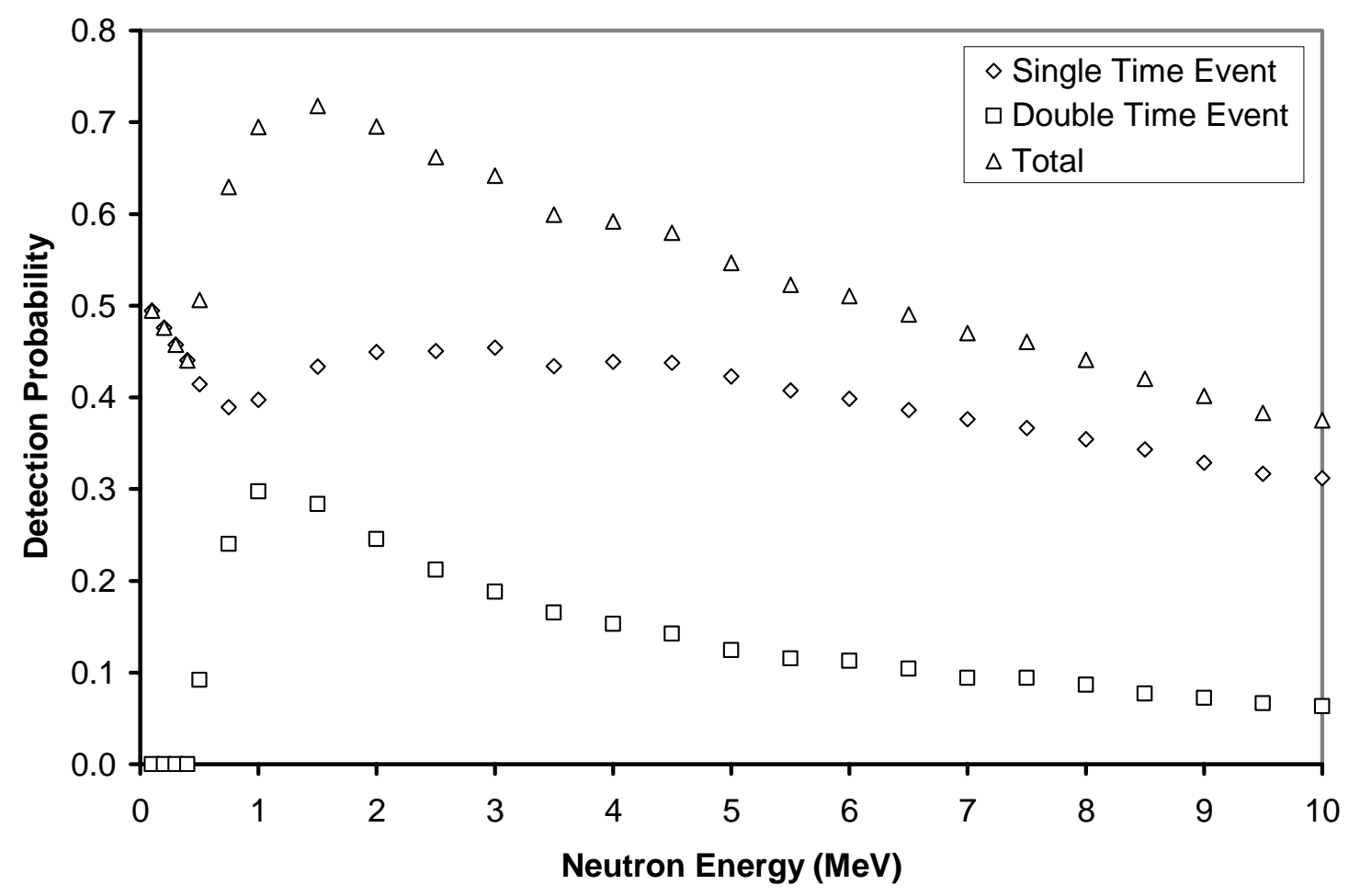

(b) 
Figure 2.

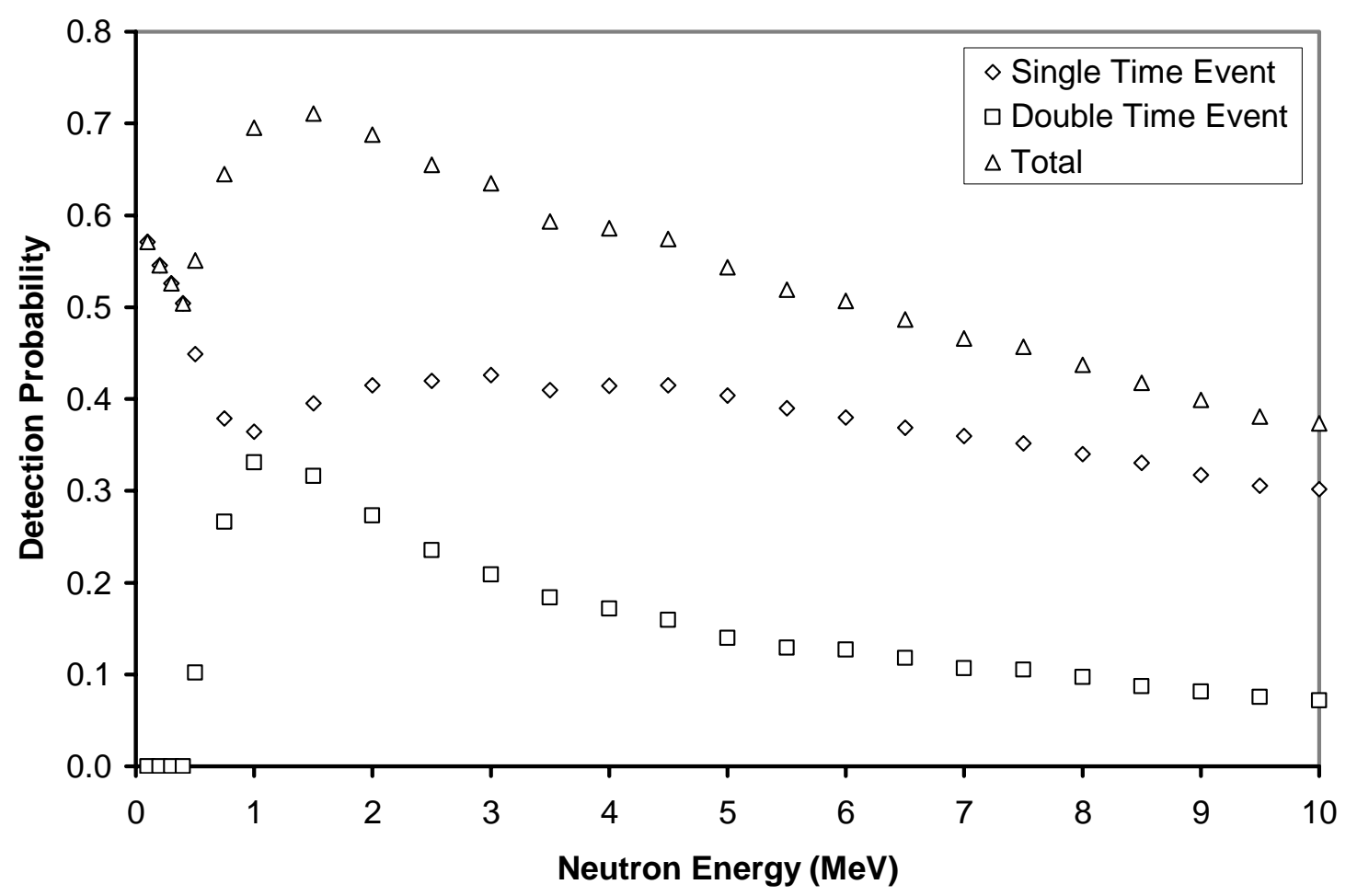

(c)

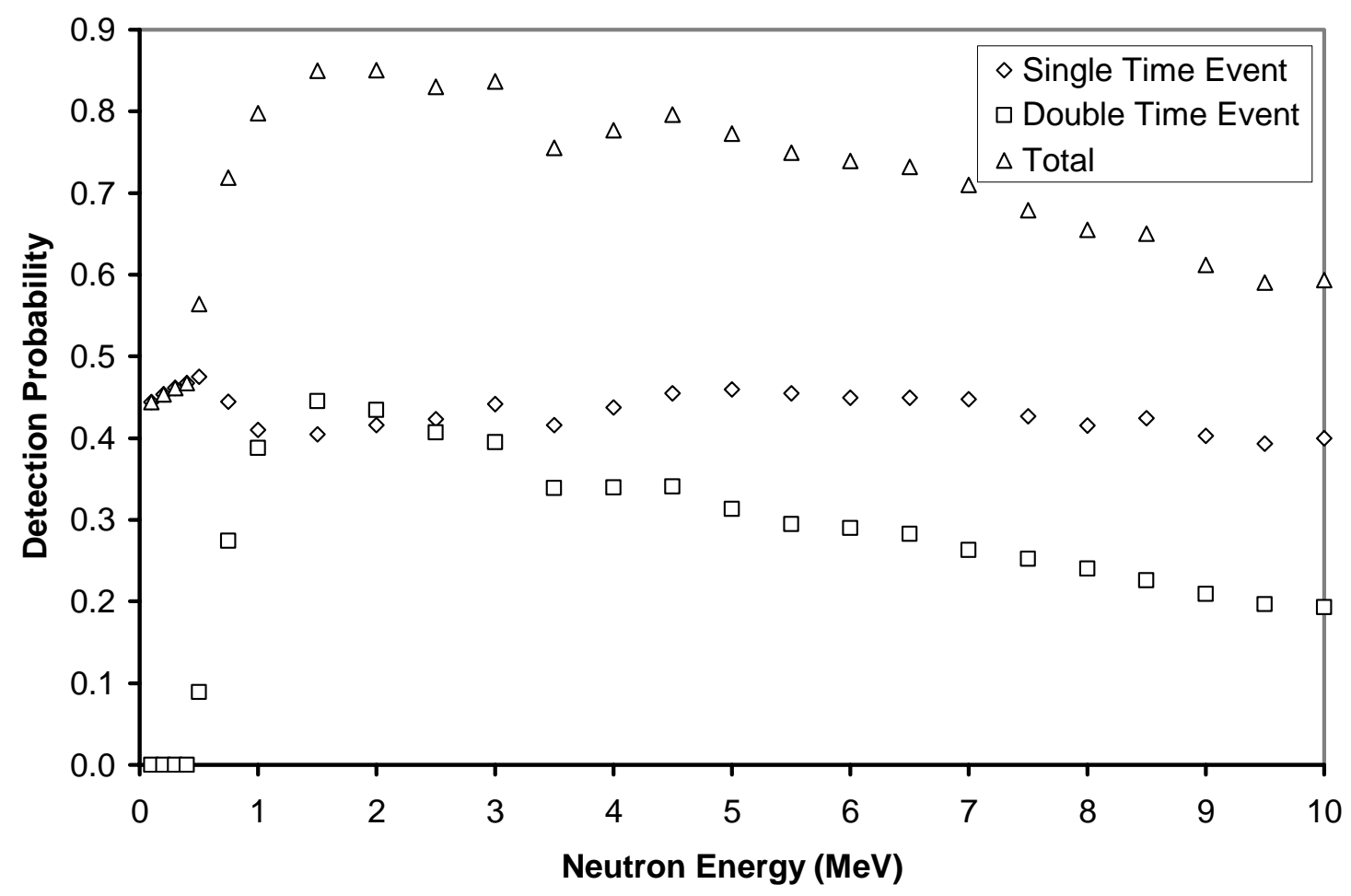

(d) 
Figure 2.

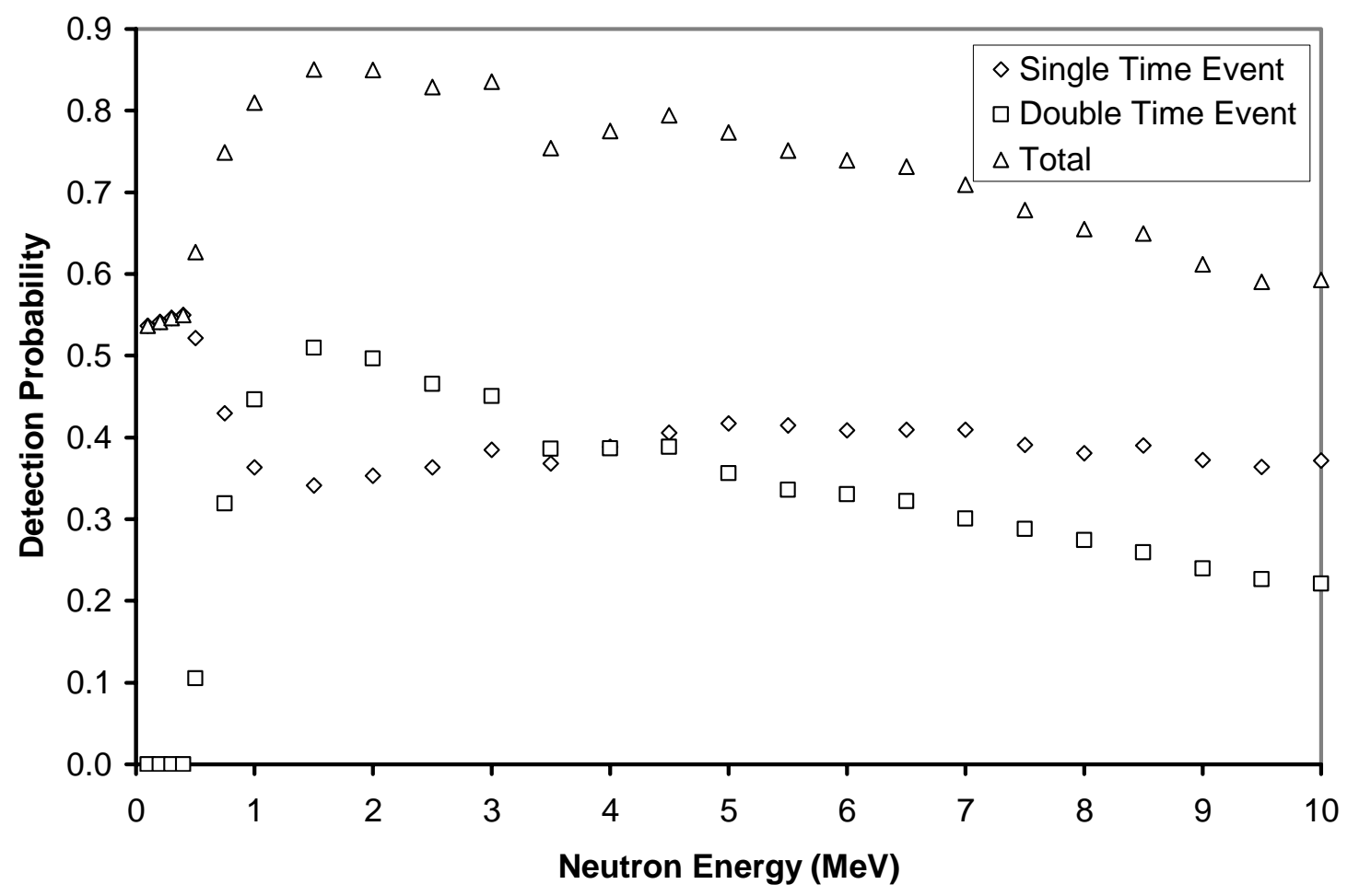

(e)

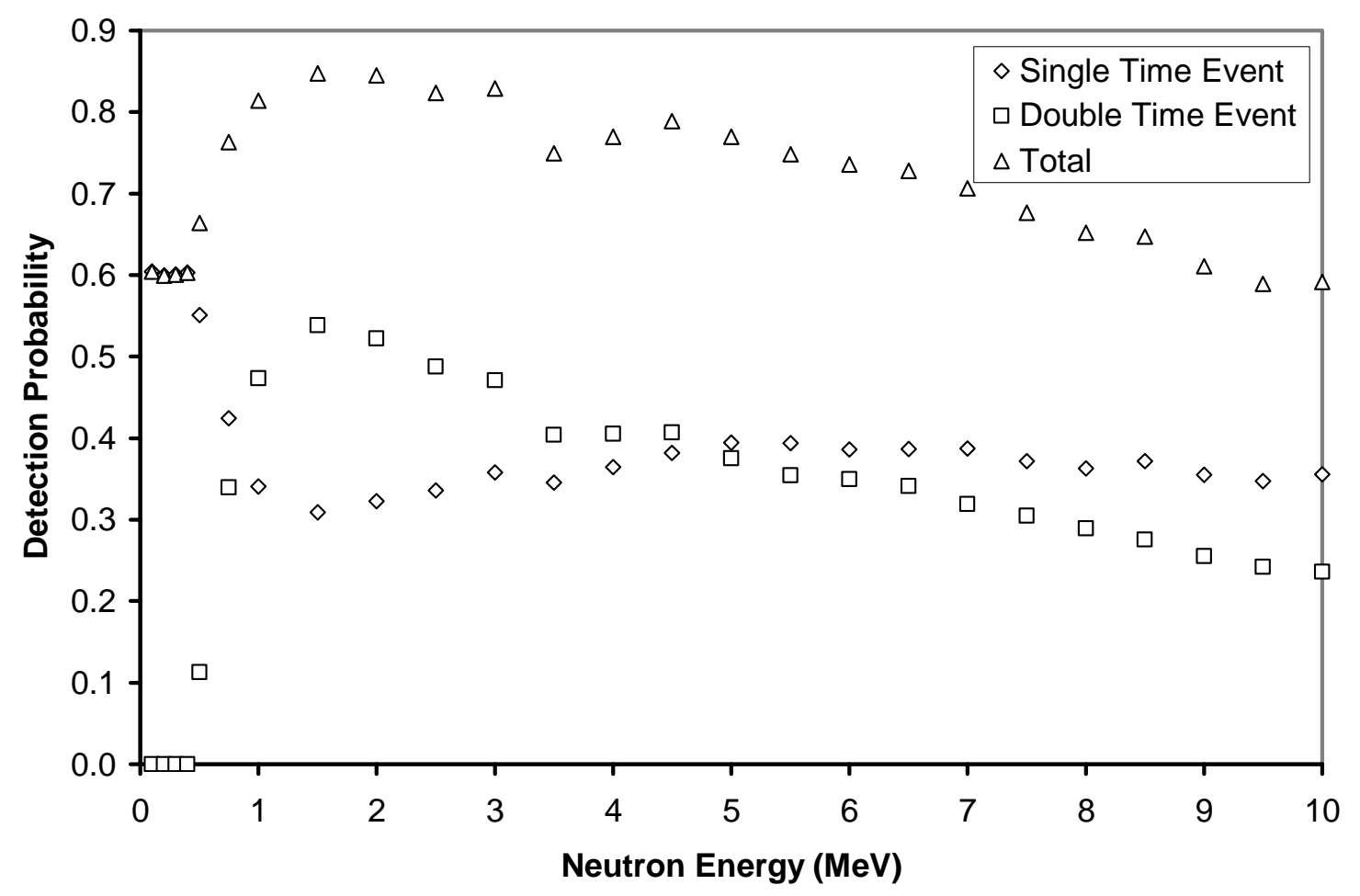

(f) 
Figure 2.

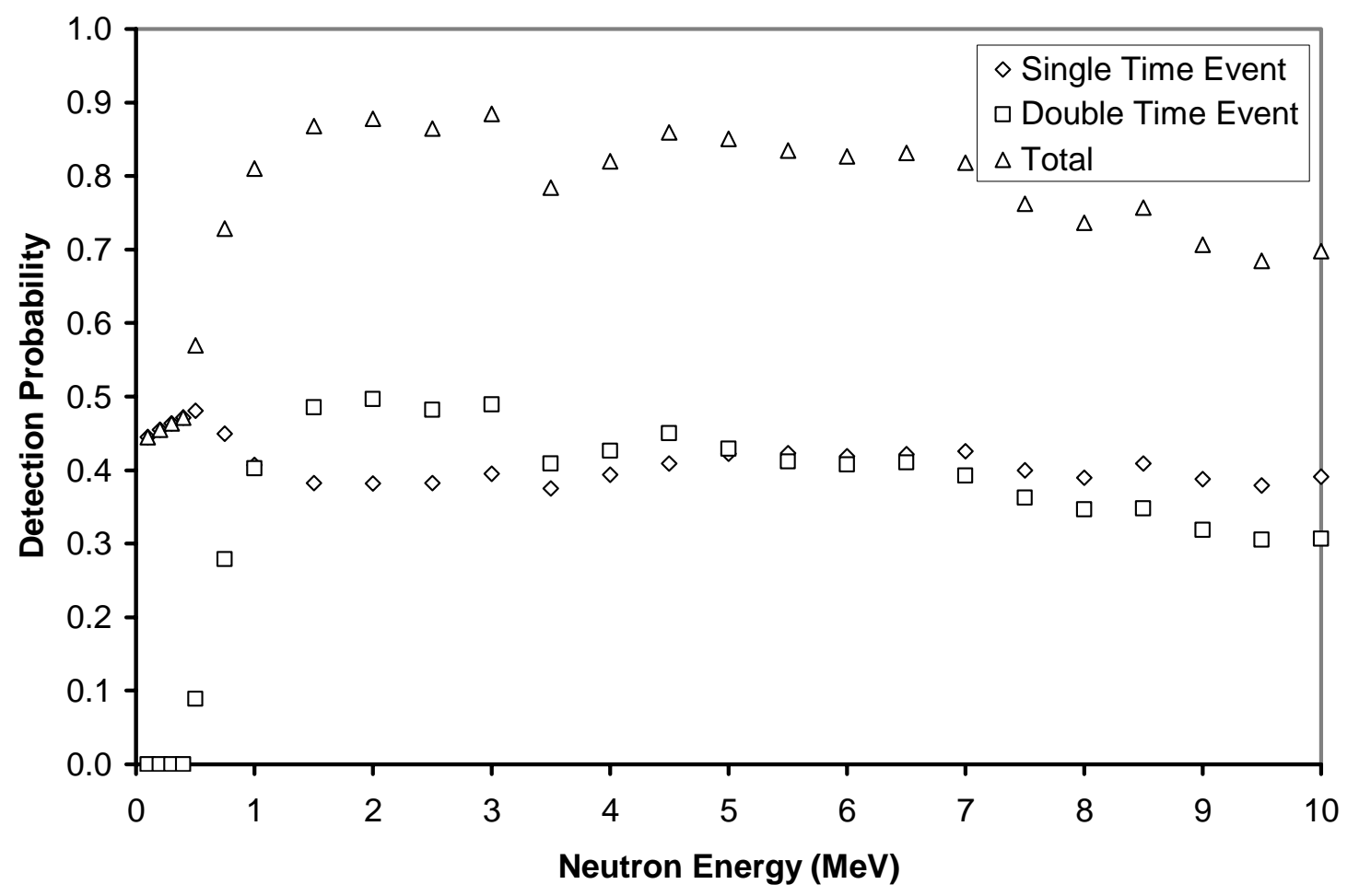

(g)

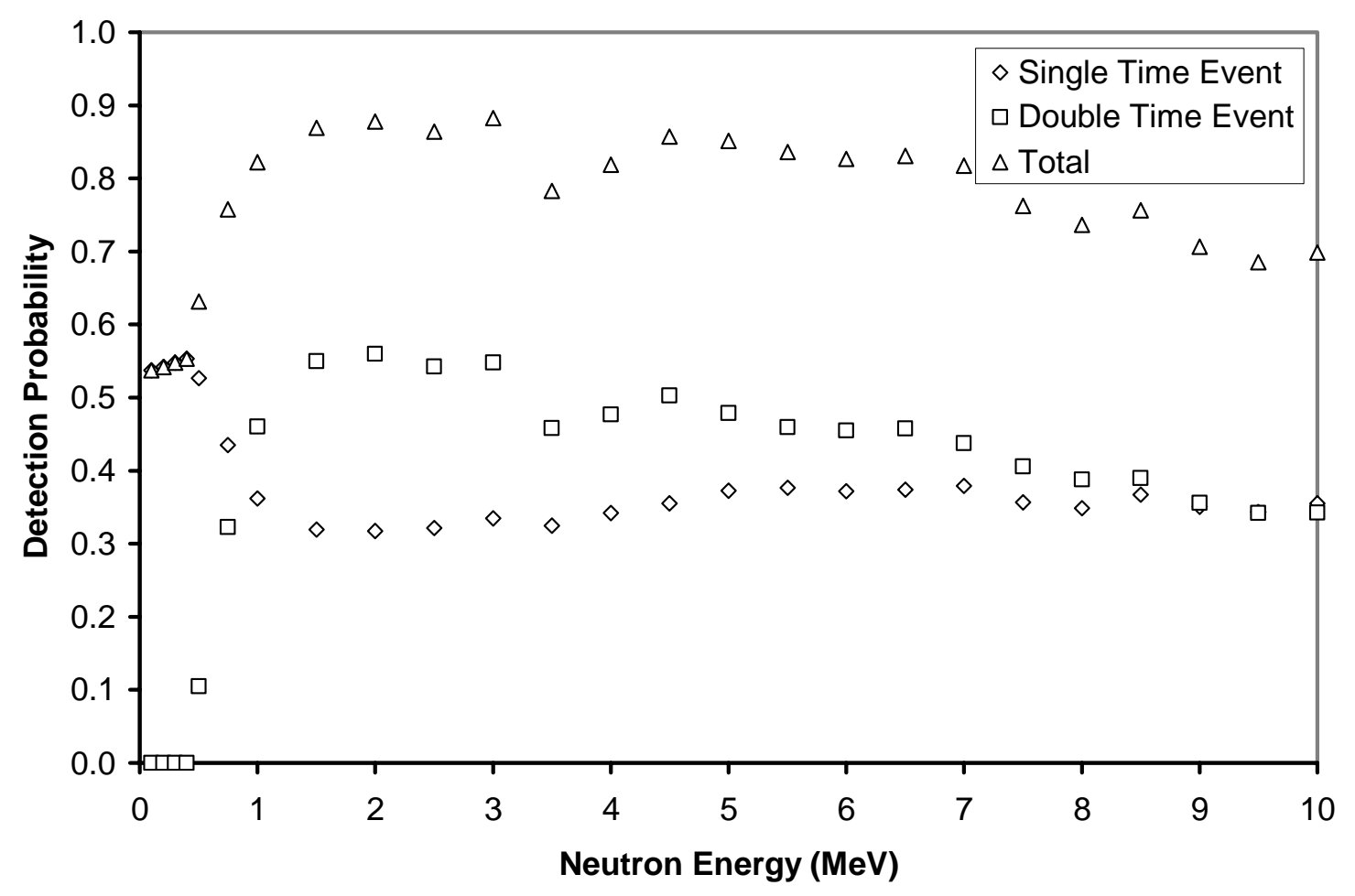

(h) 
Figure 2.

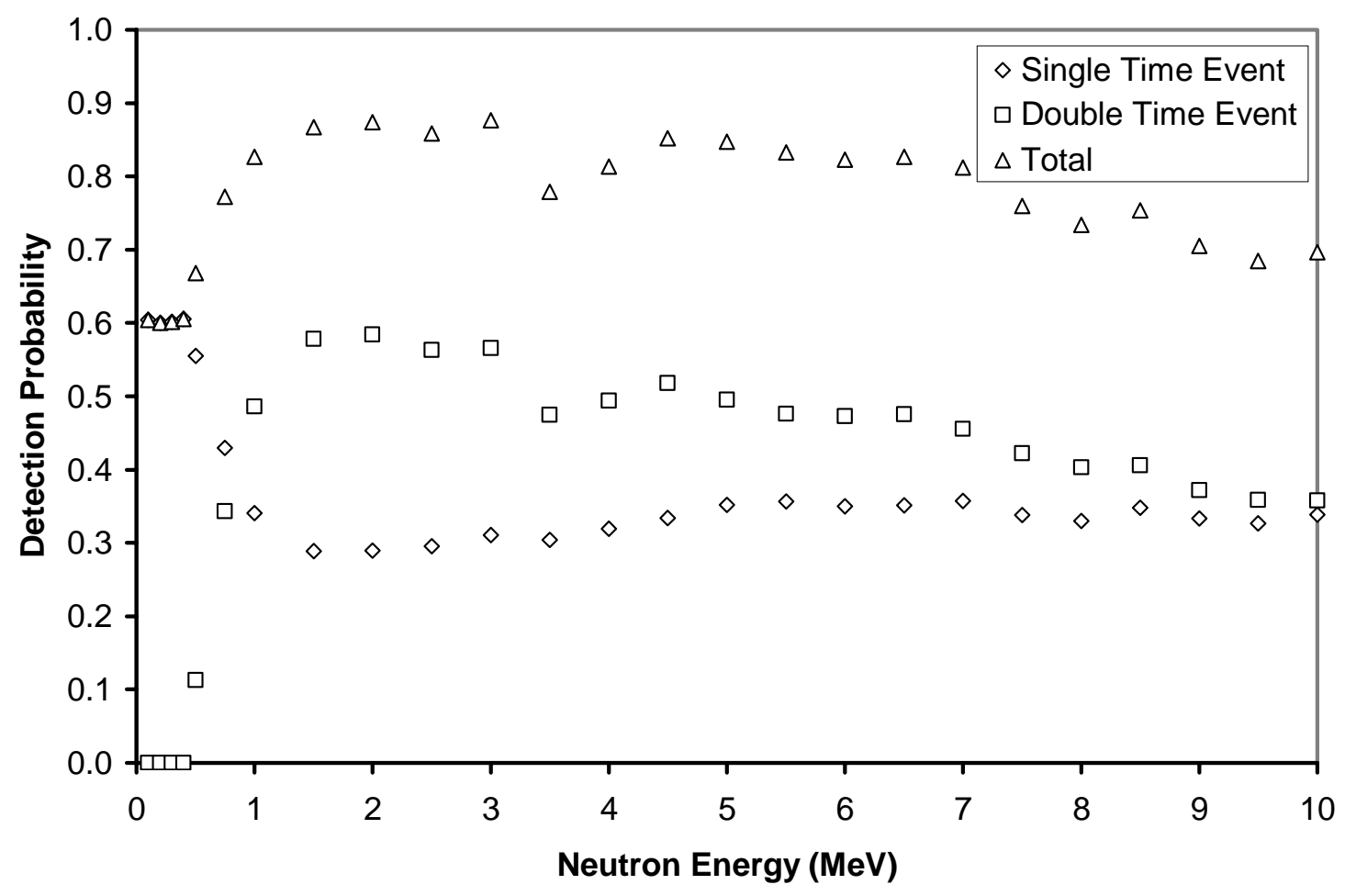

(i) 
Figure 3.

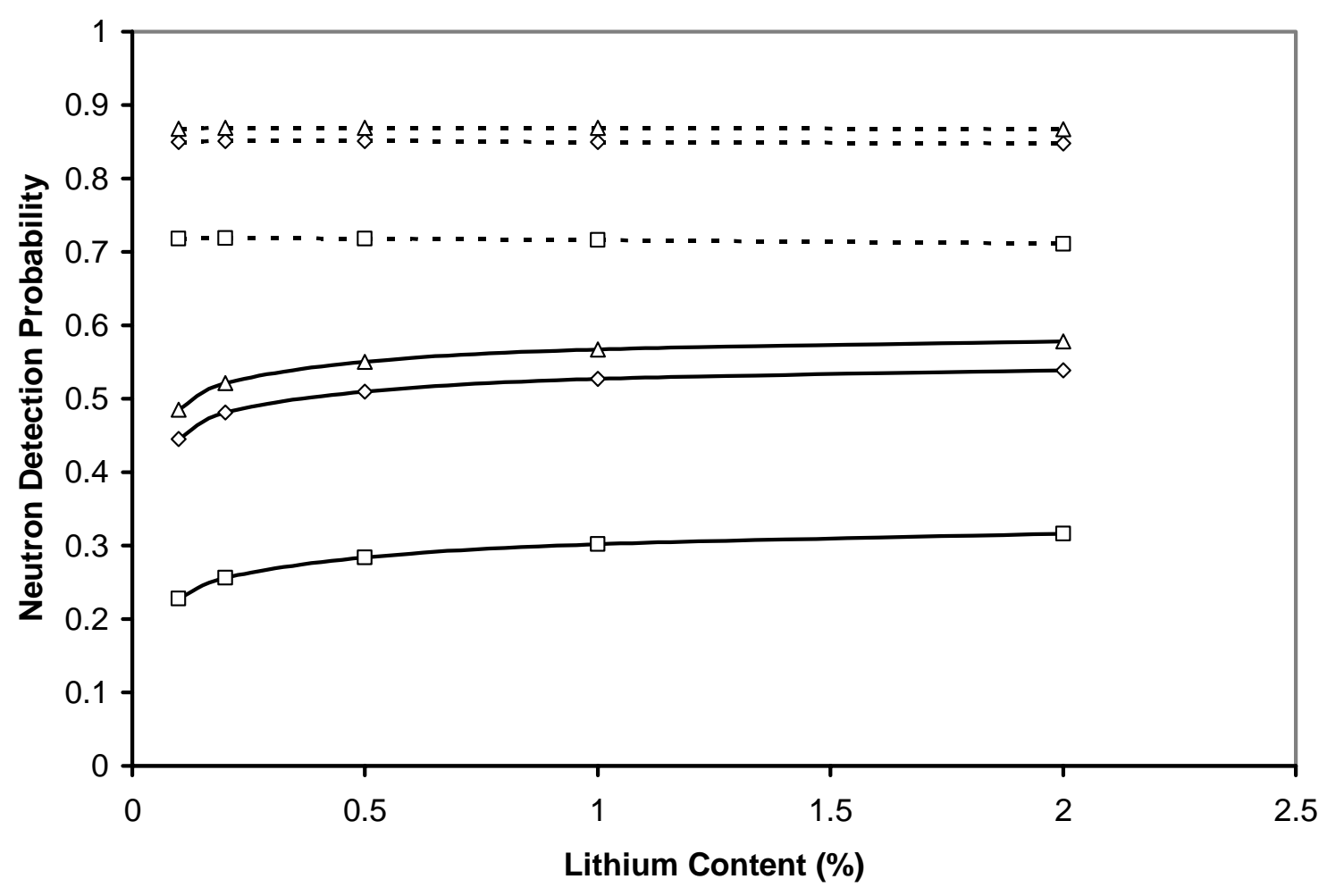


Figure 4.

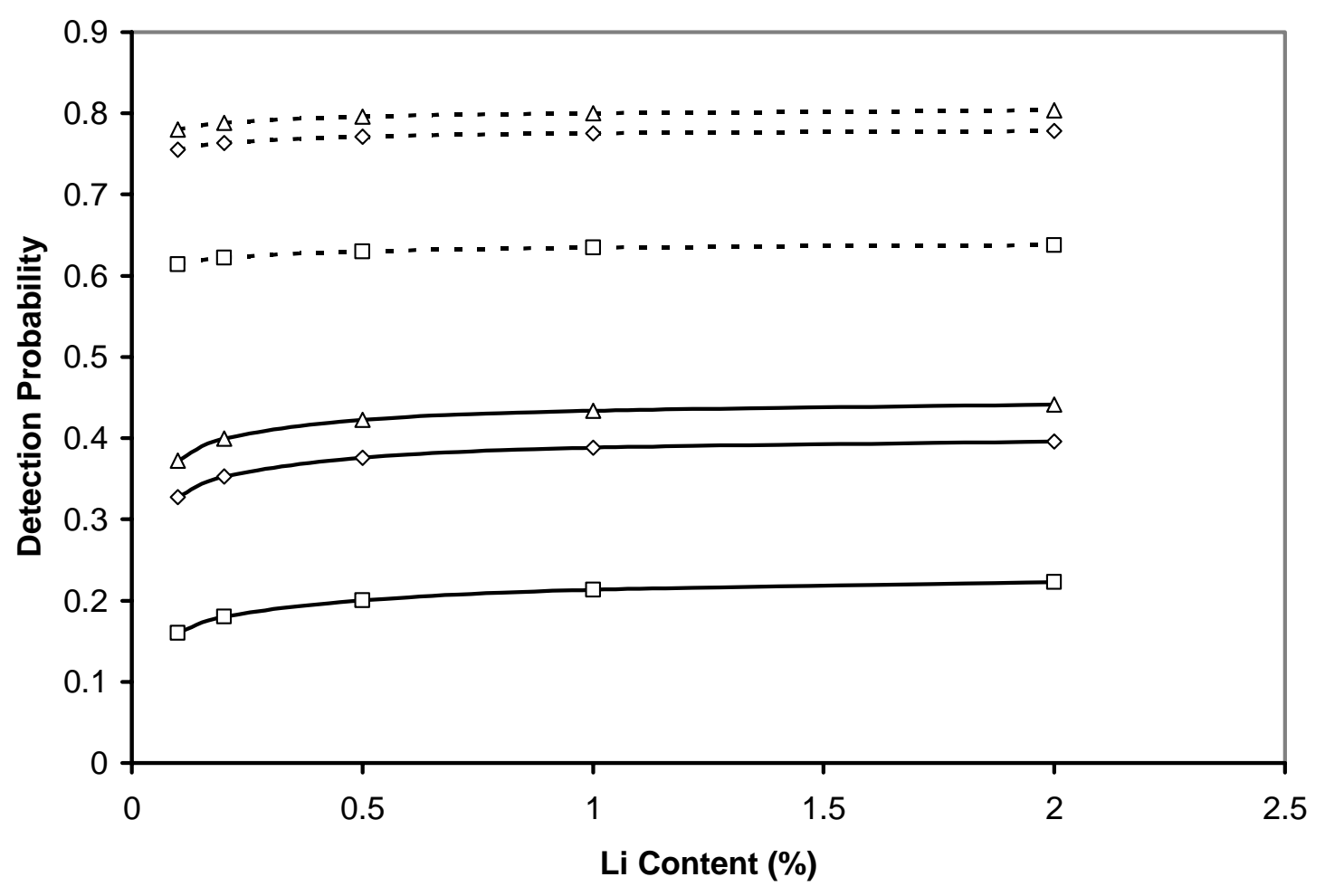


Figure 5.

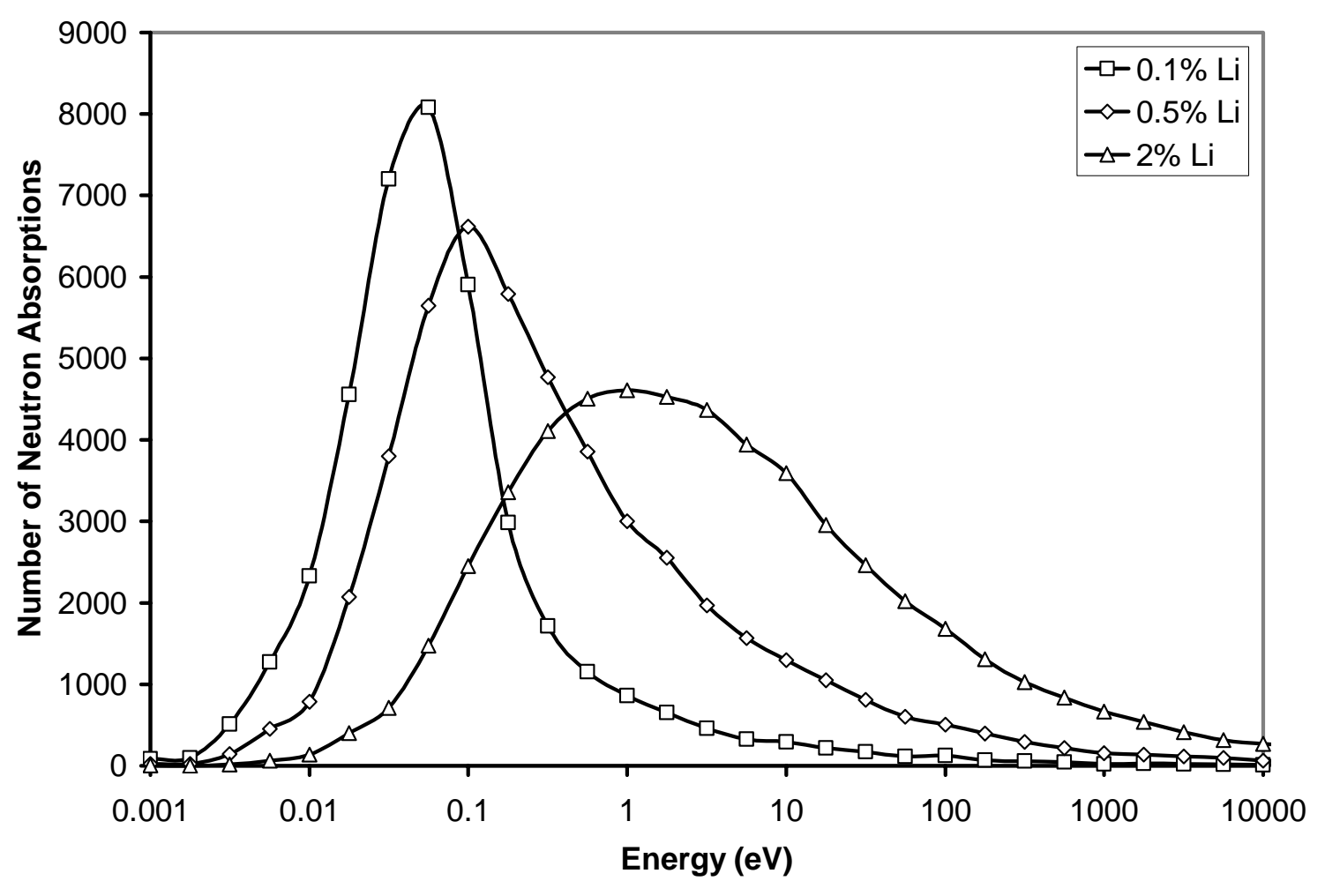


Figure 6.

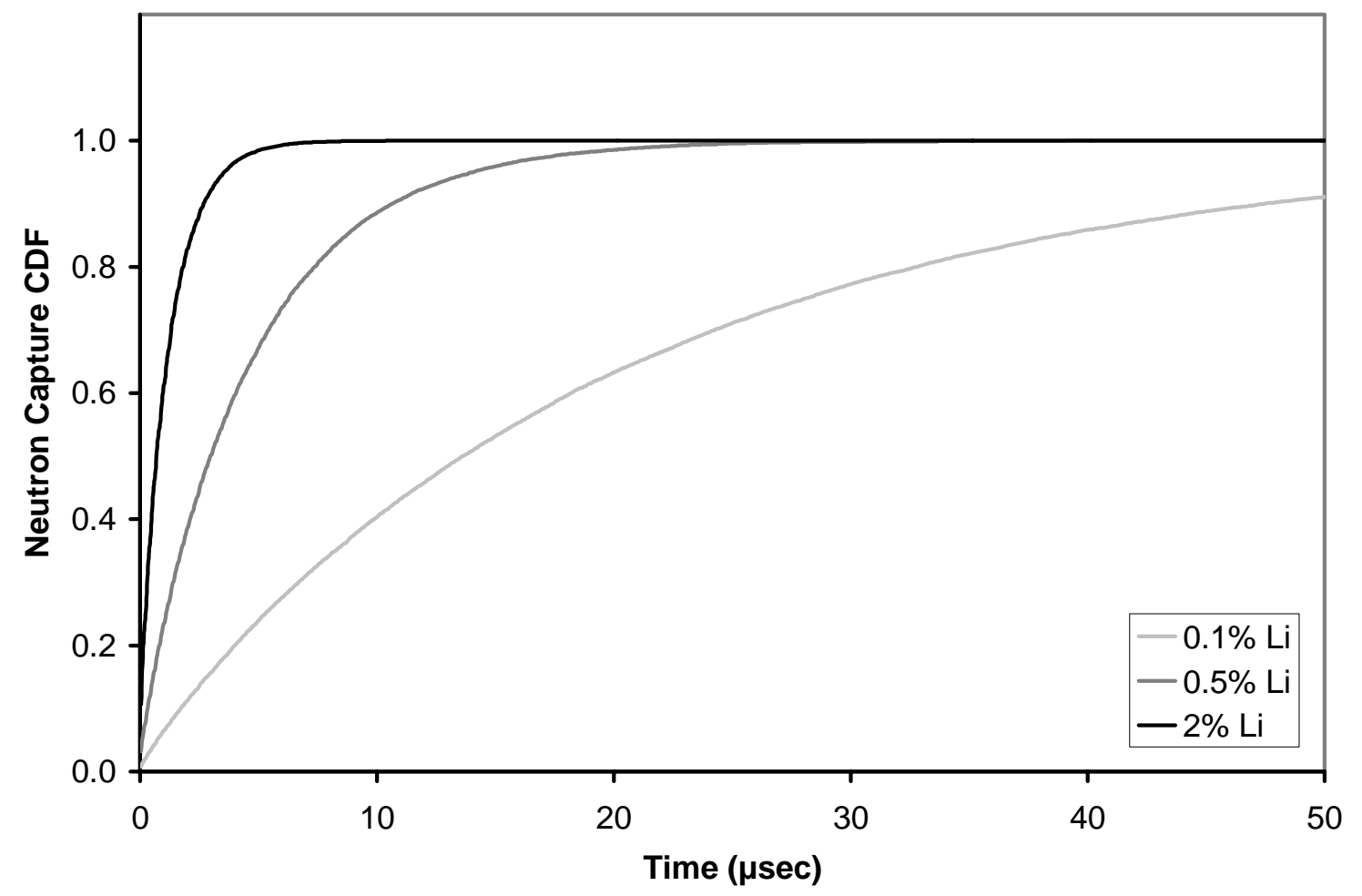


Figure 7.

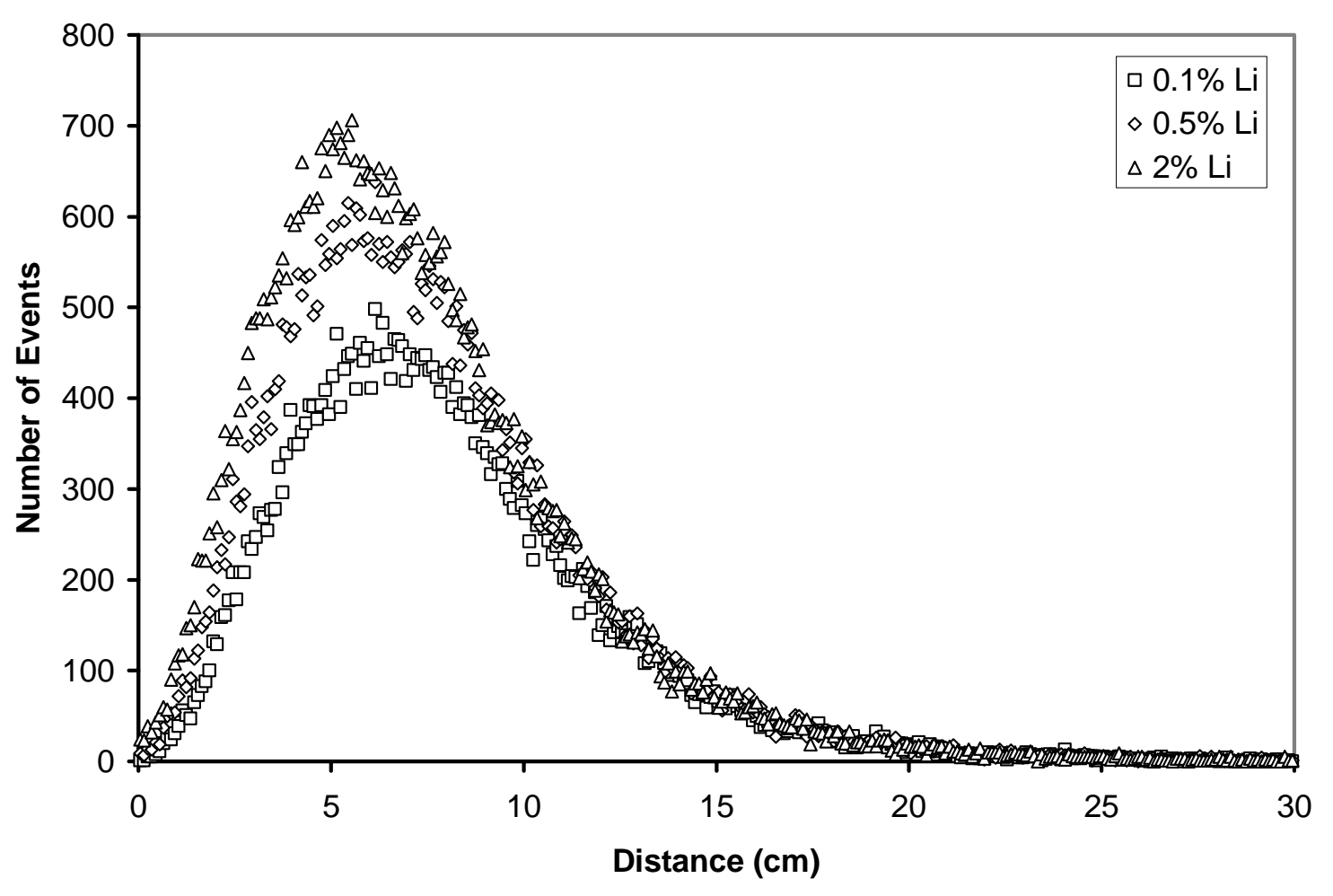

(a)

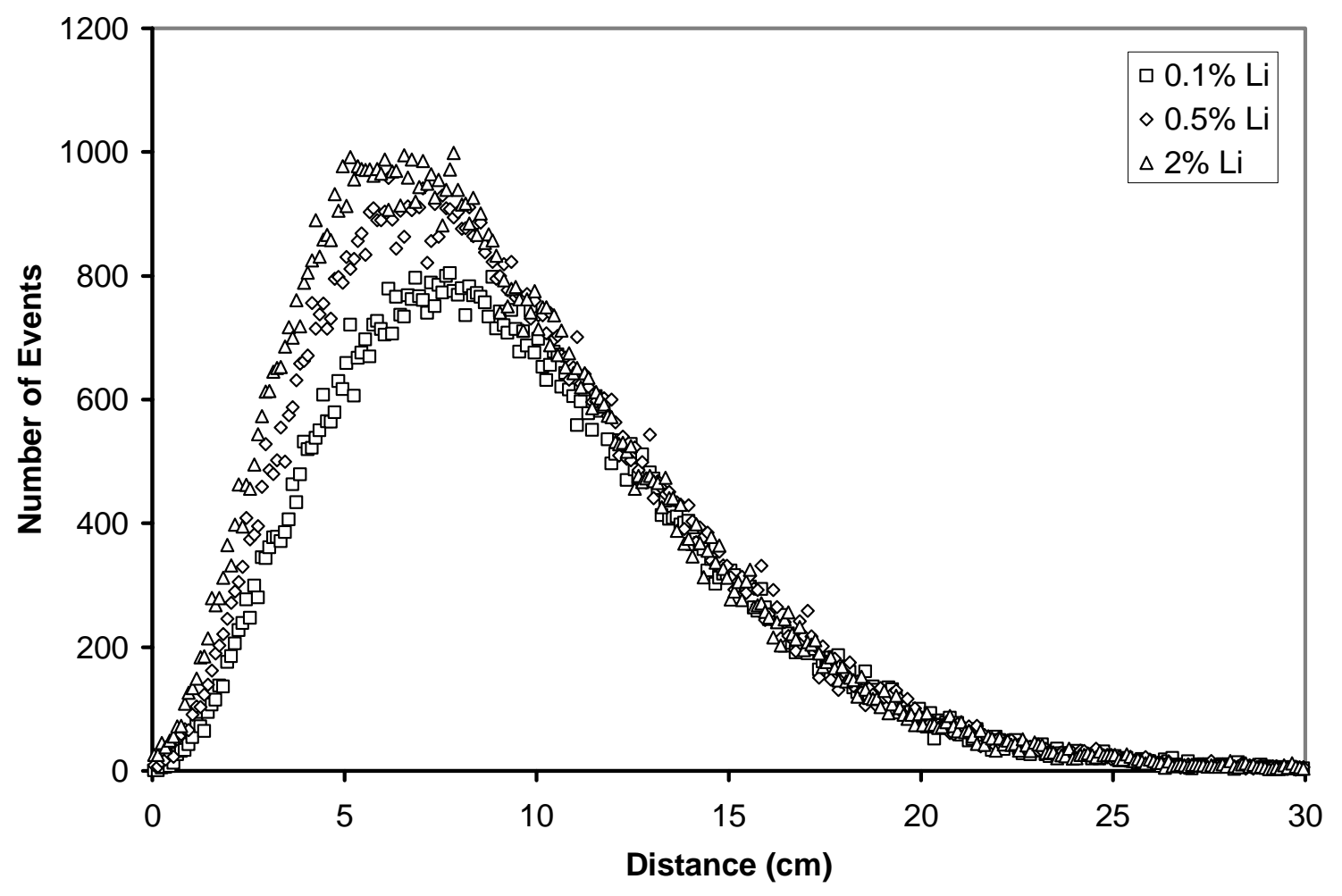

(b) 
Figure 7.

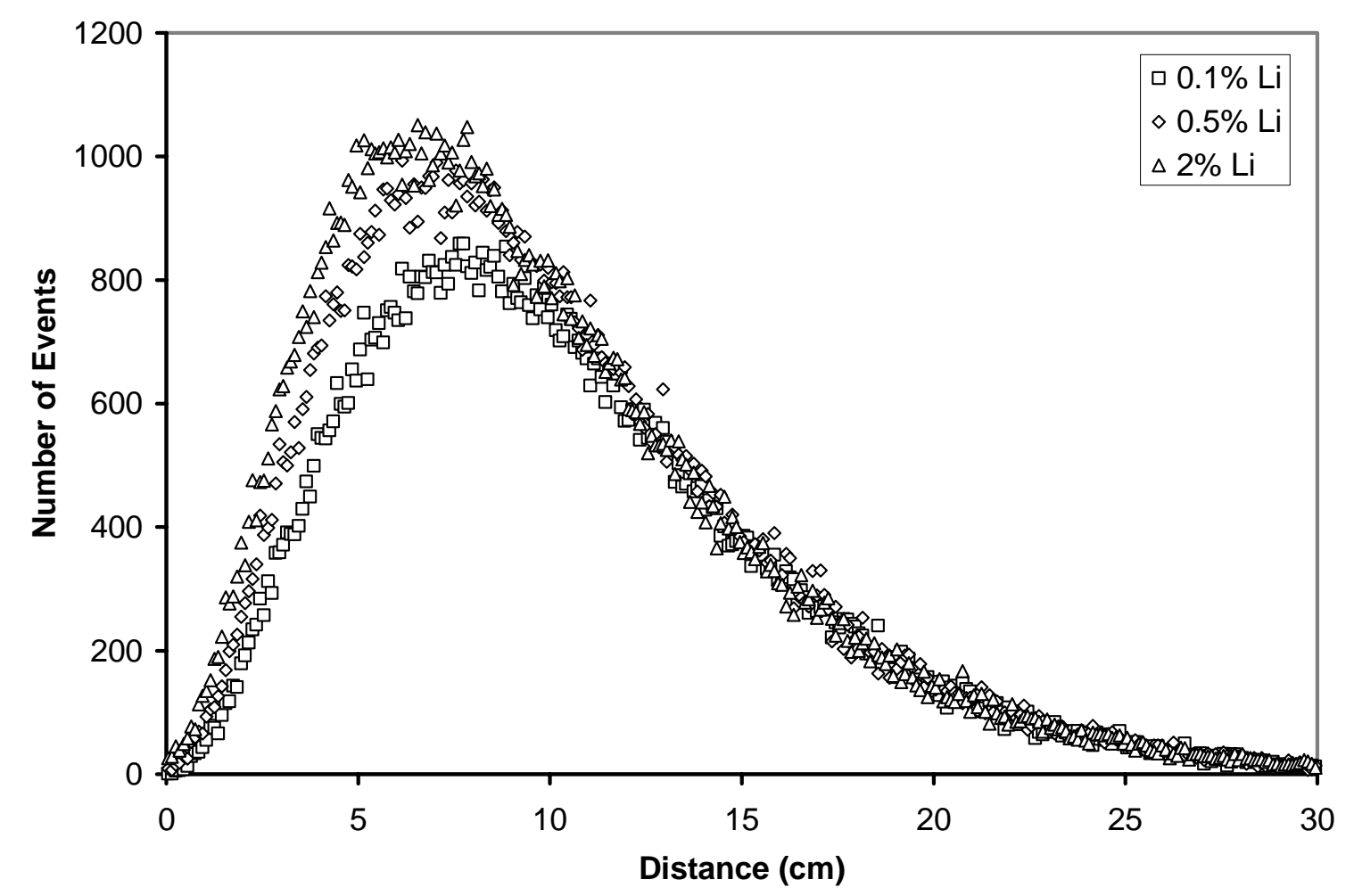

(c) 
Figure 8.

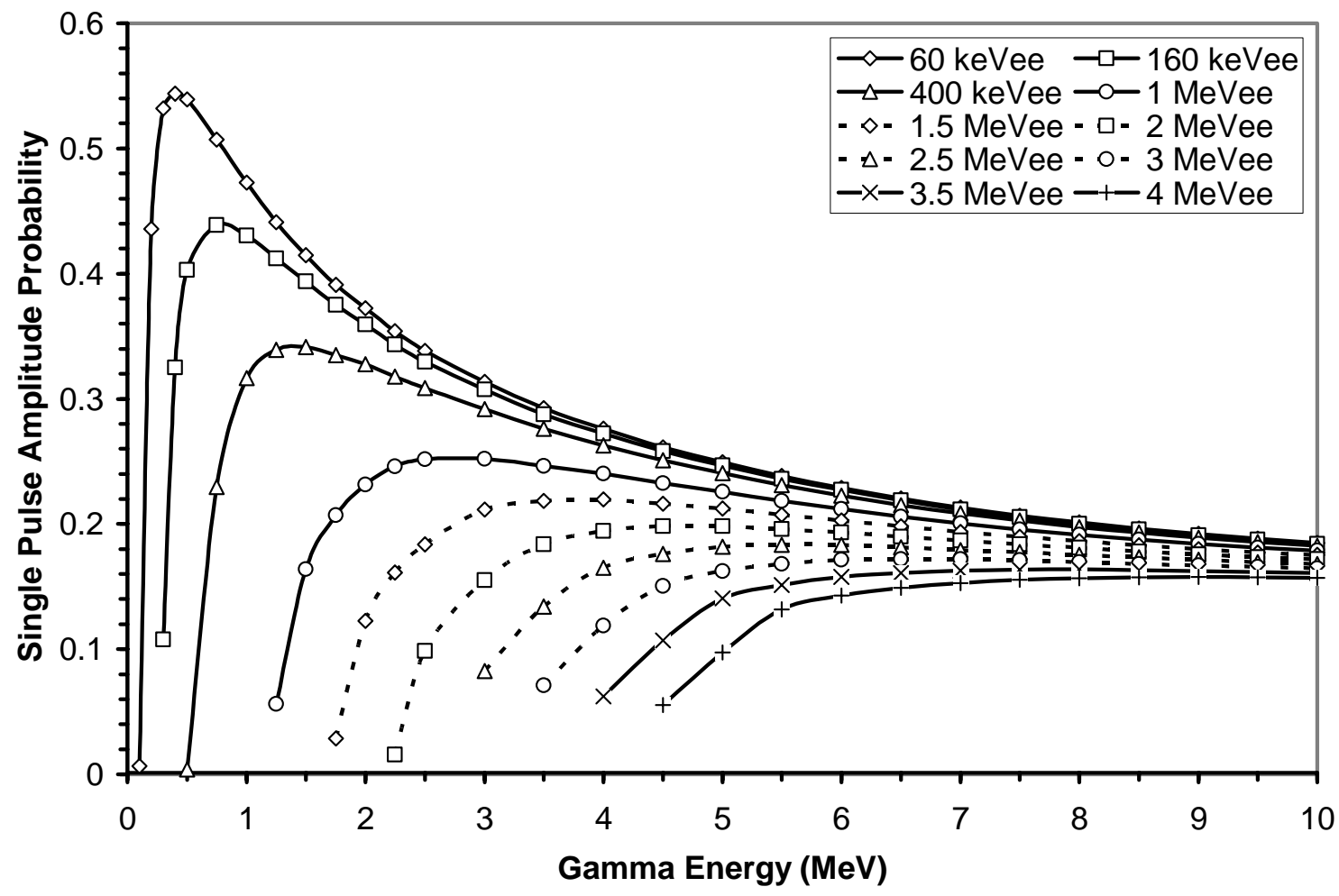

(a)

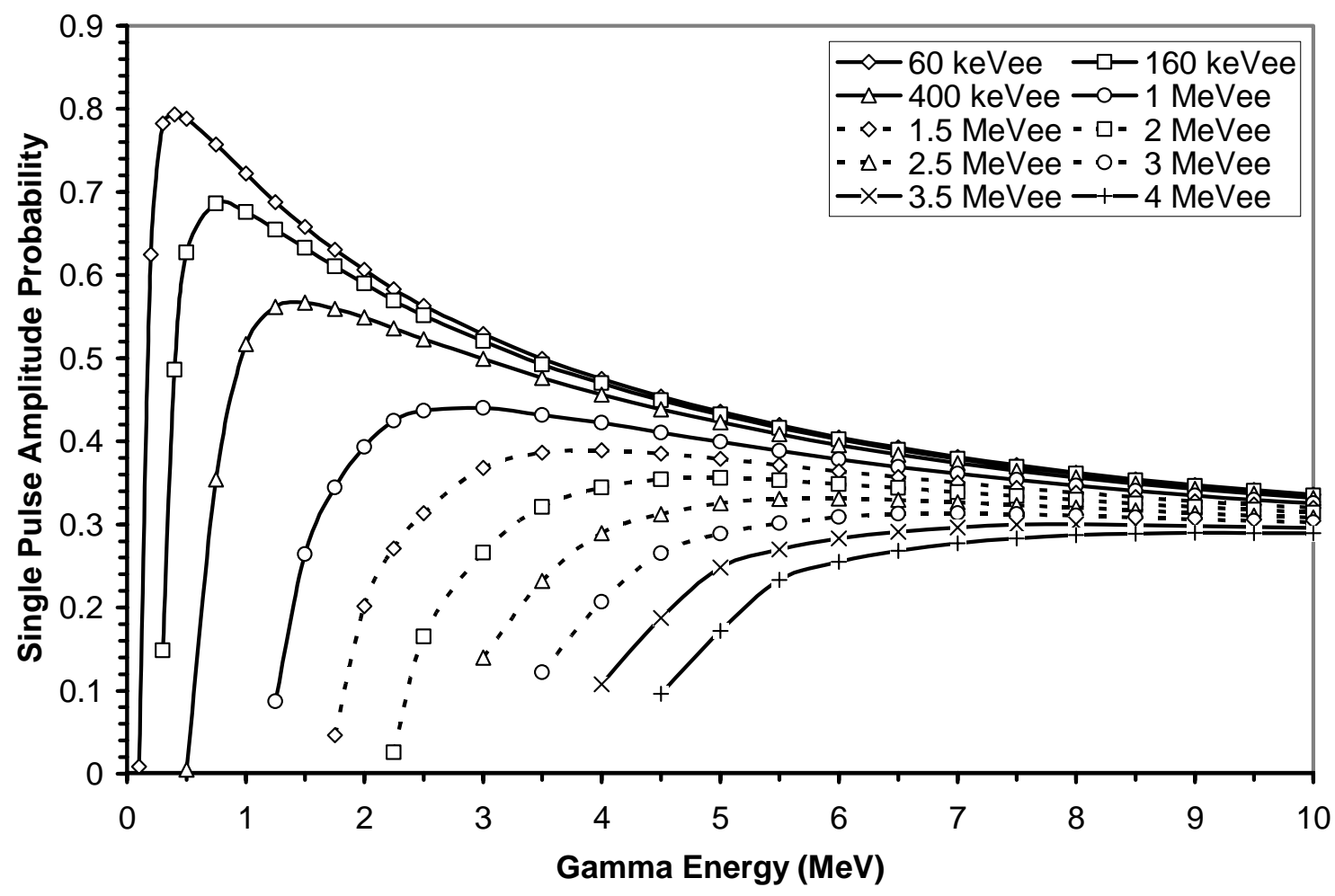

(b) 
Figure 8.

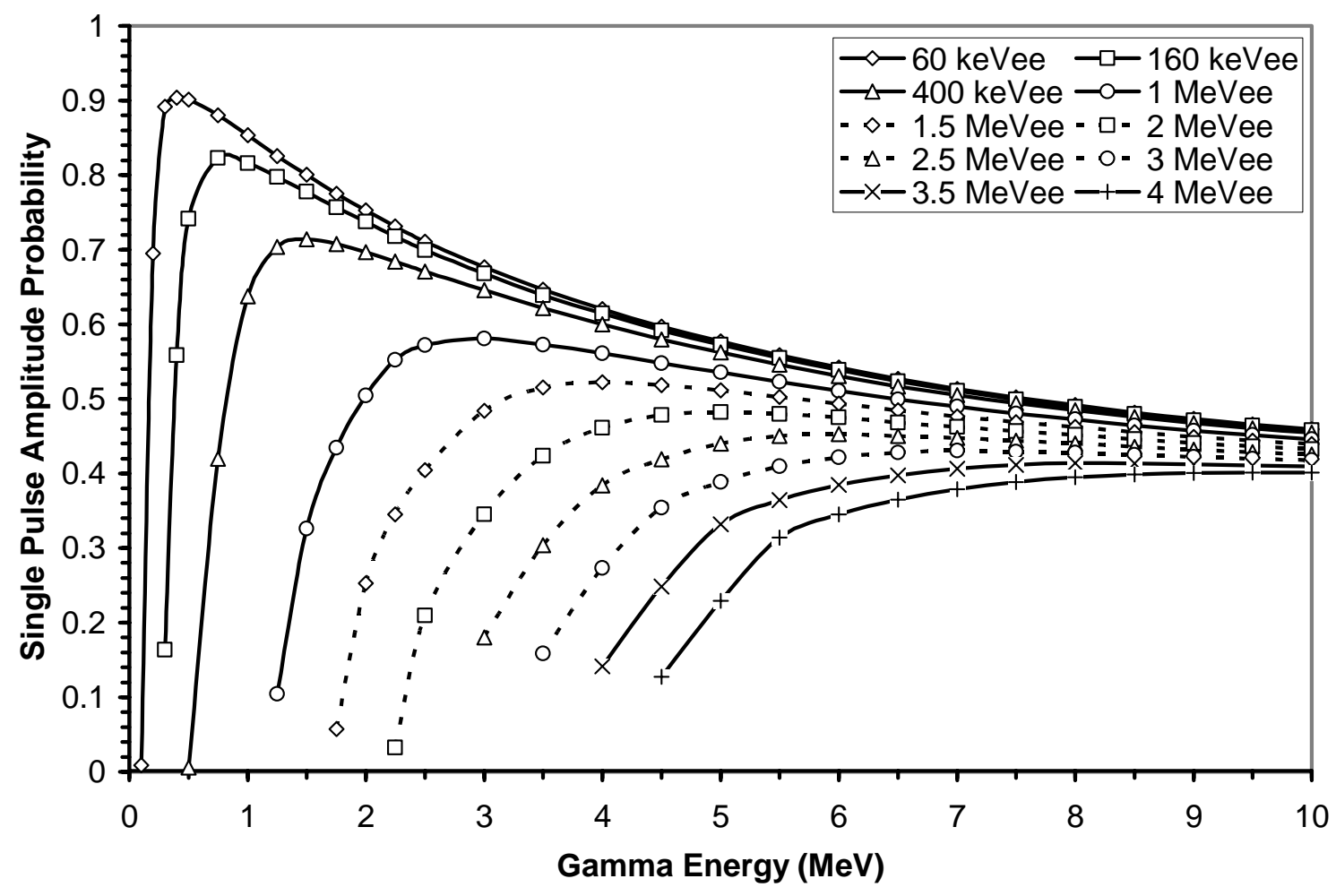

(c)

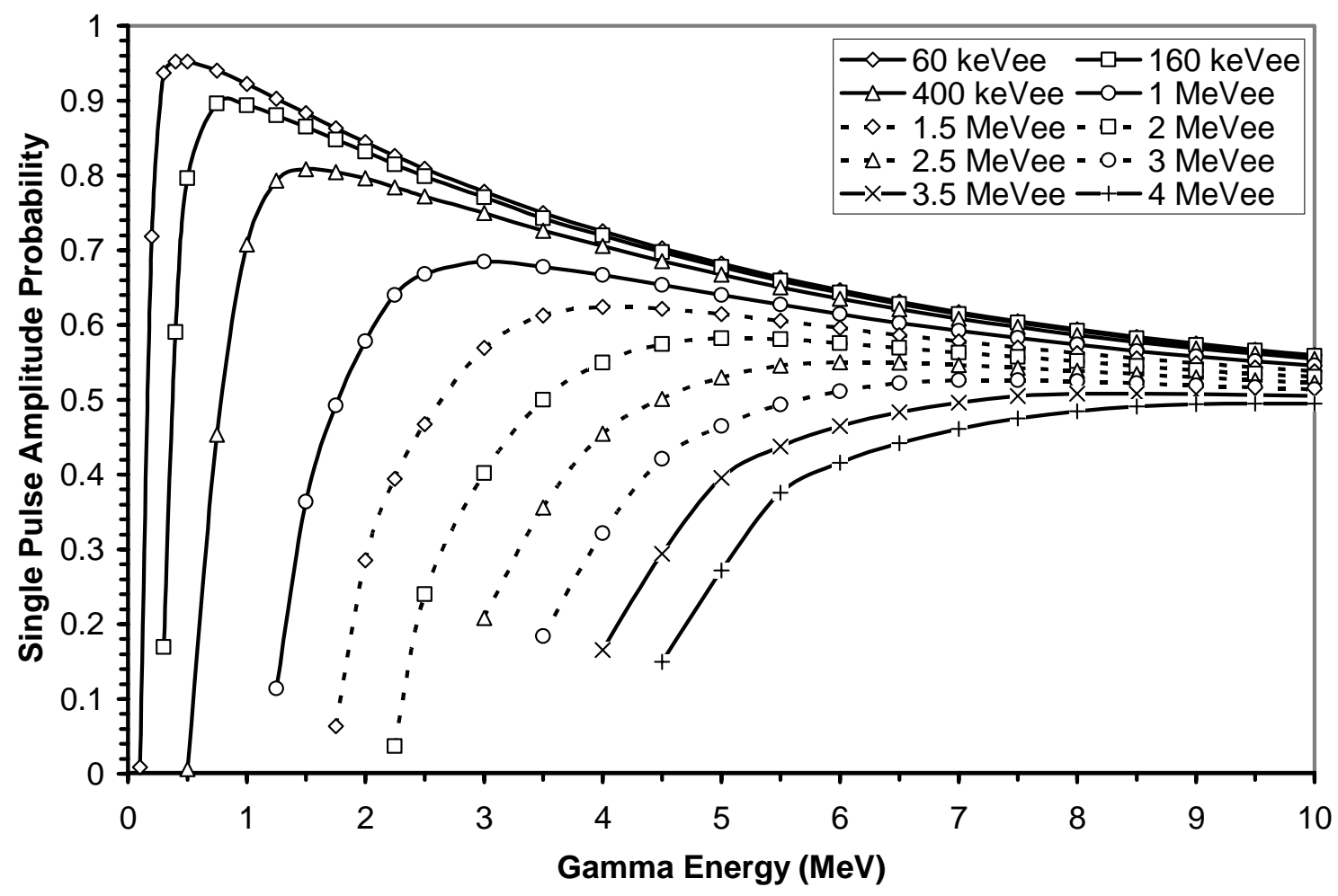

(d) 
Figure 9.

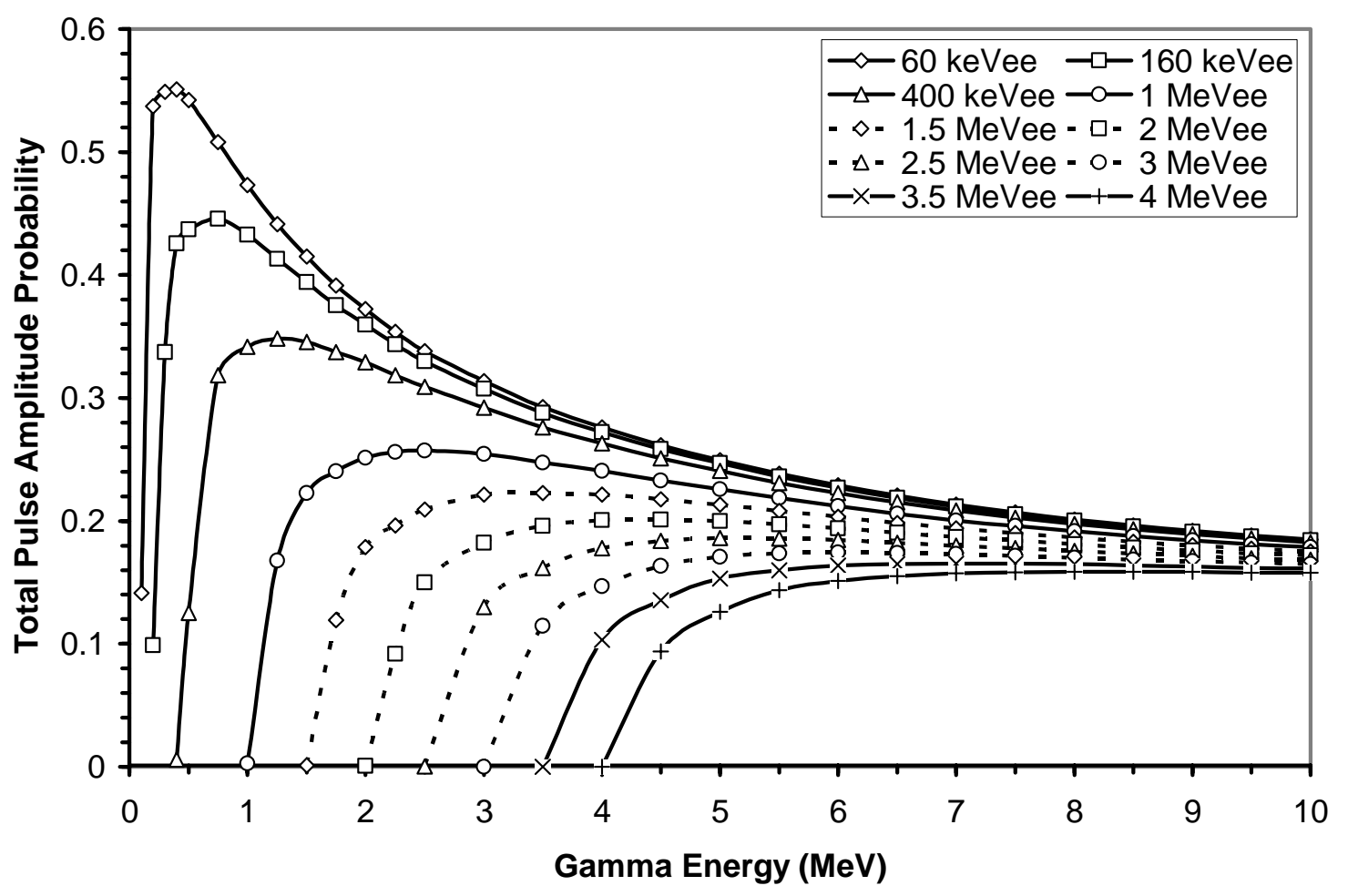

(a)

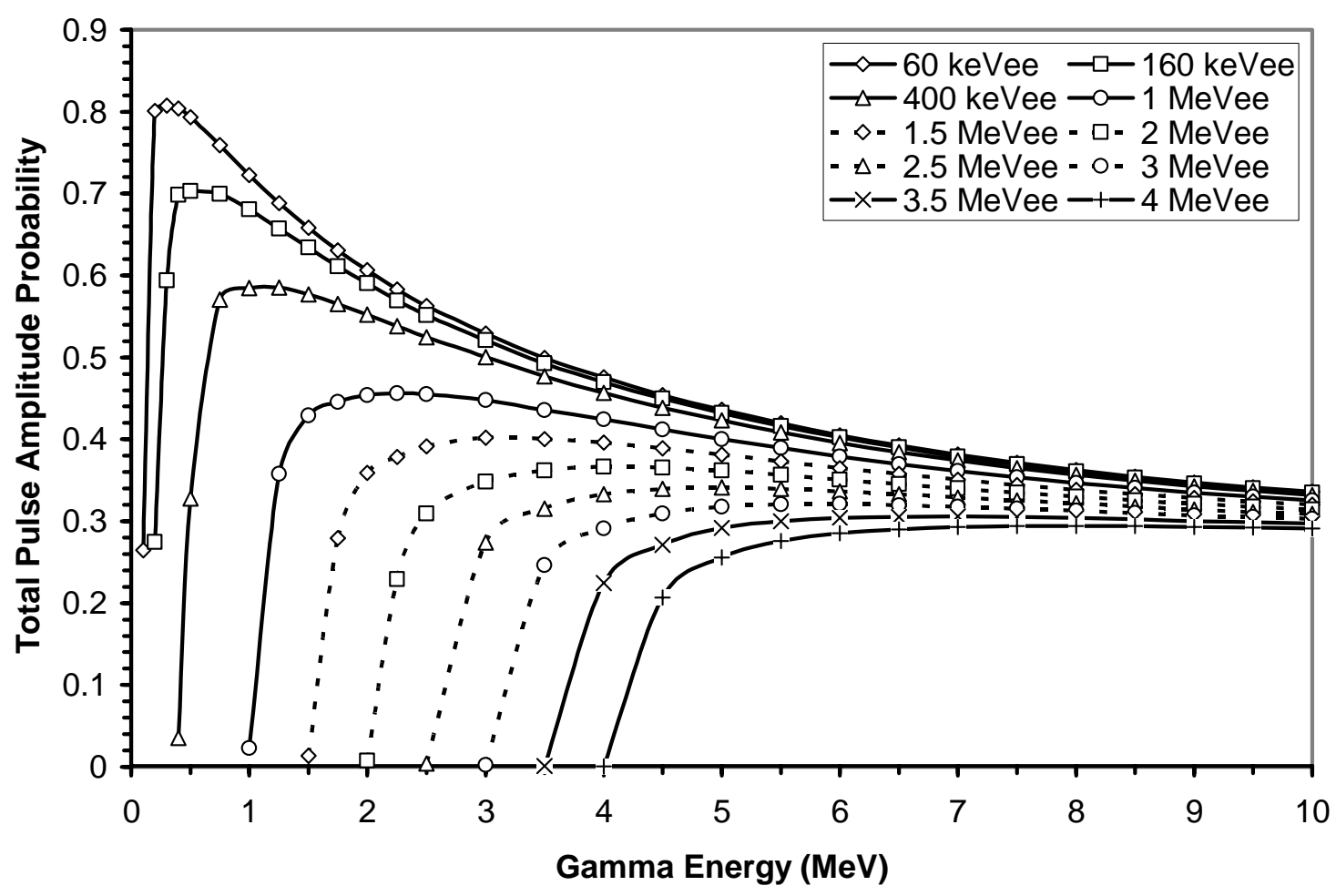

(b) 
Figure 9.

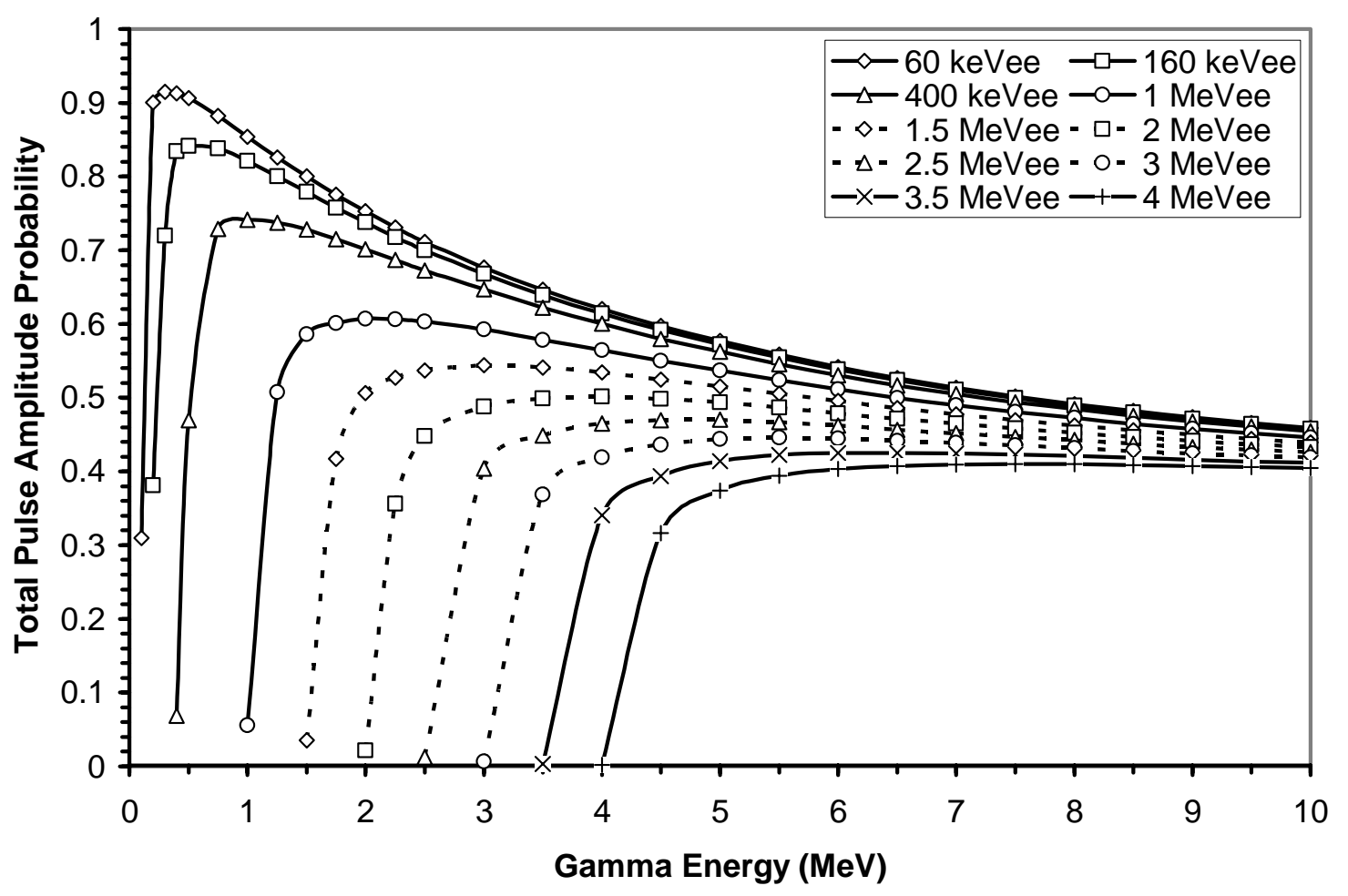

(c)

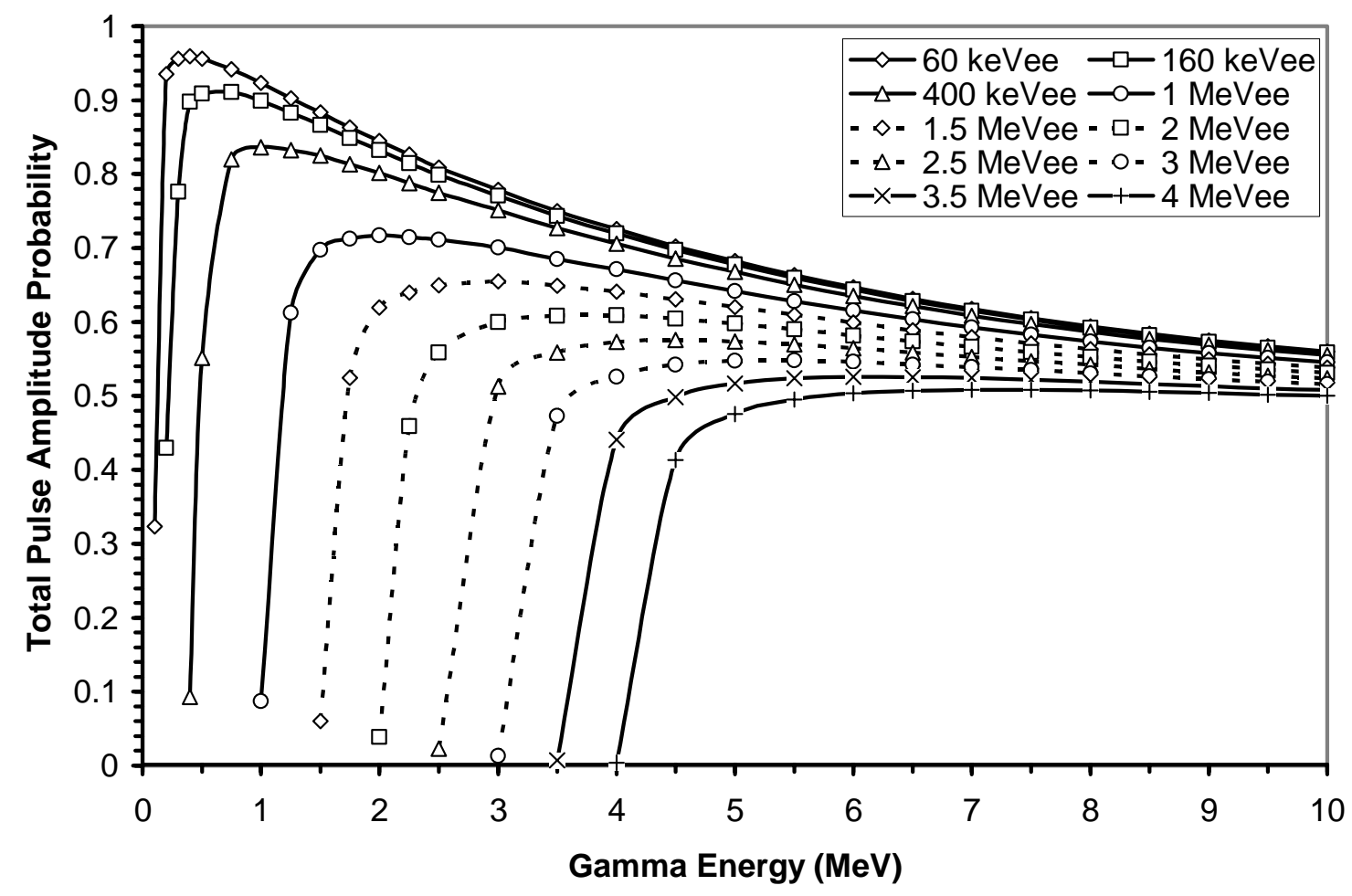

(d) 
Figure 10.

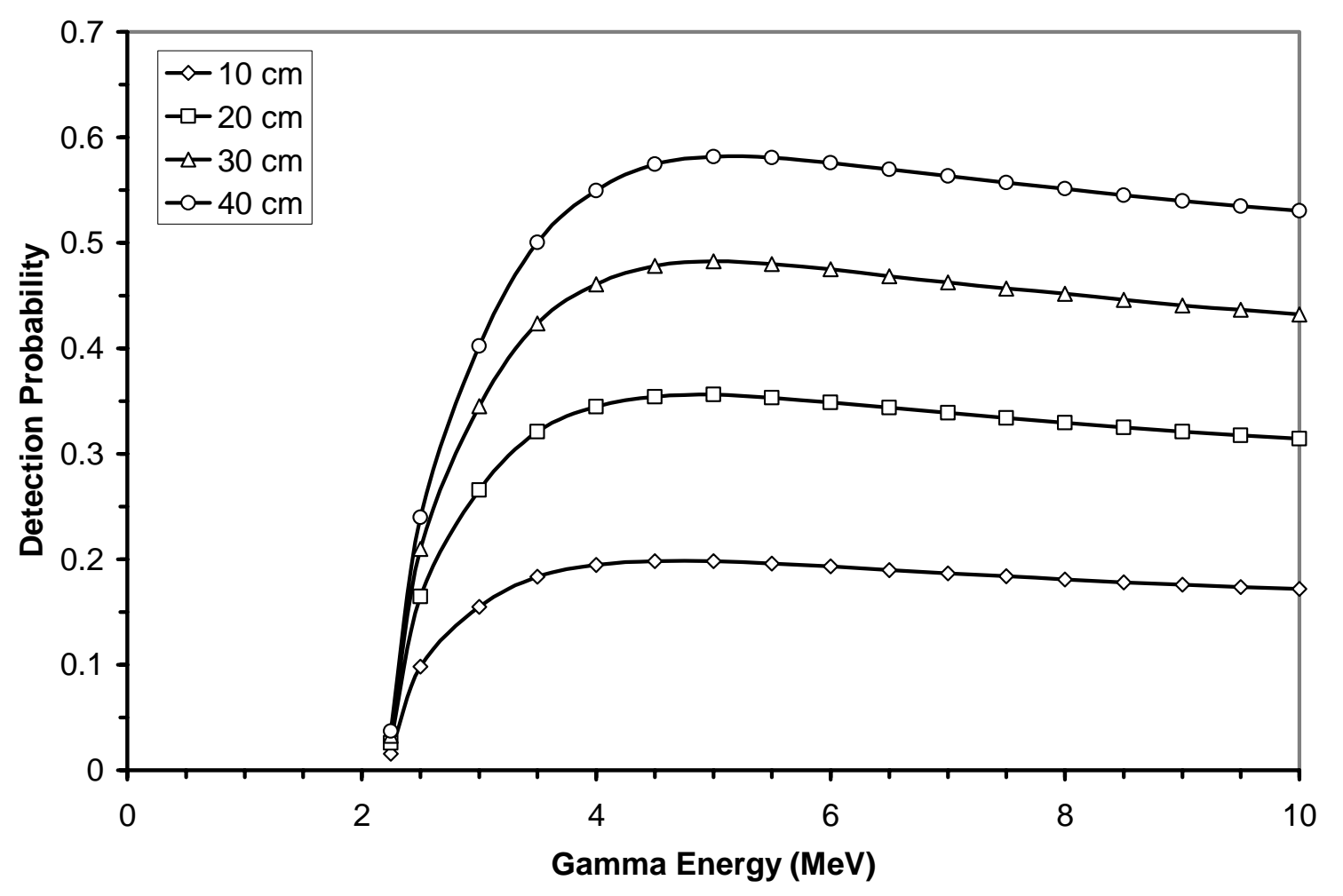

(a)

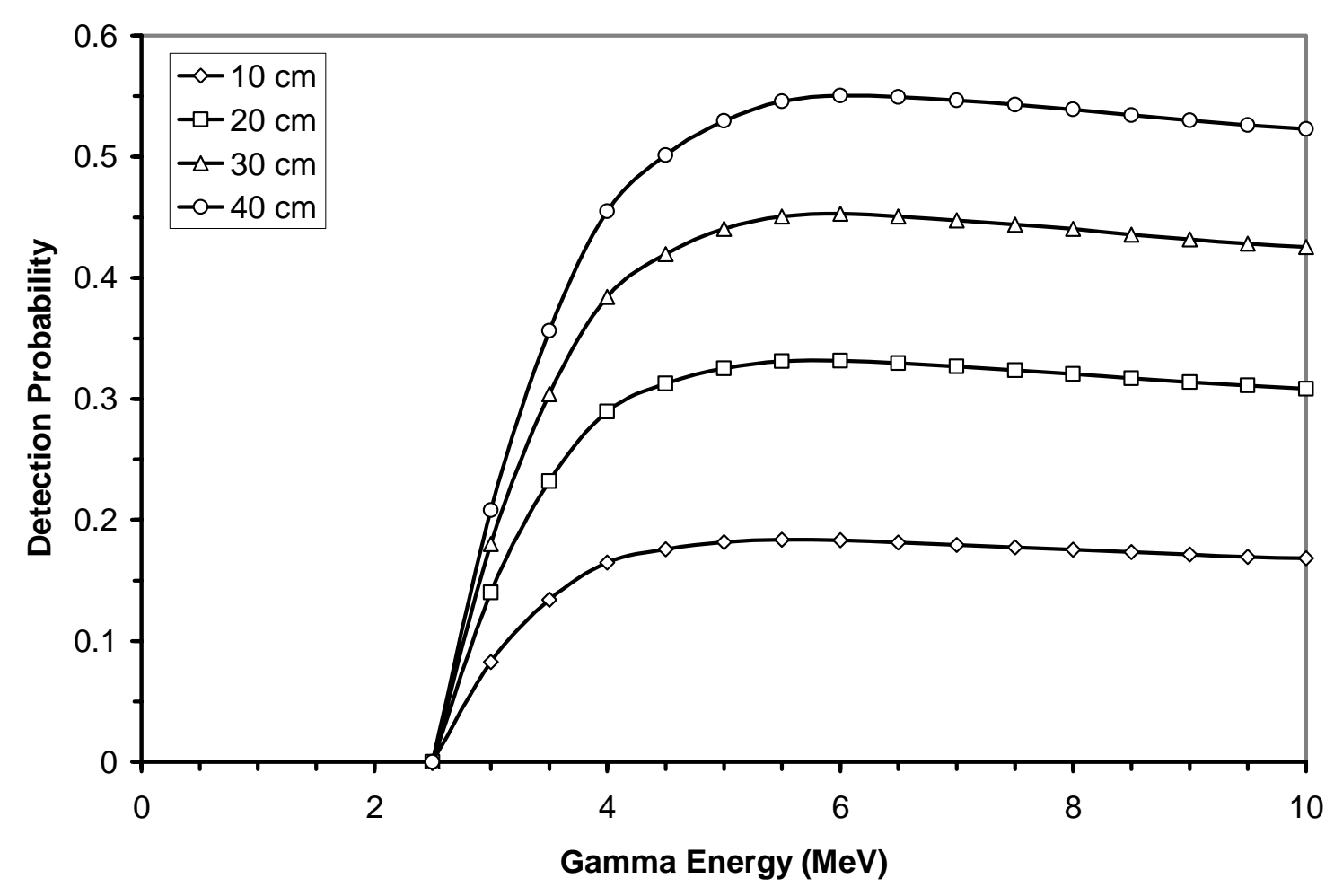

(b) 
Figure 10.

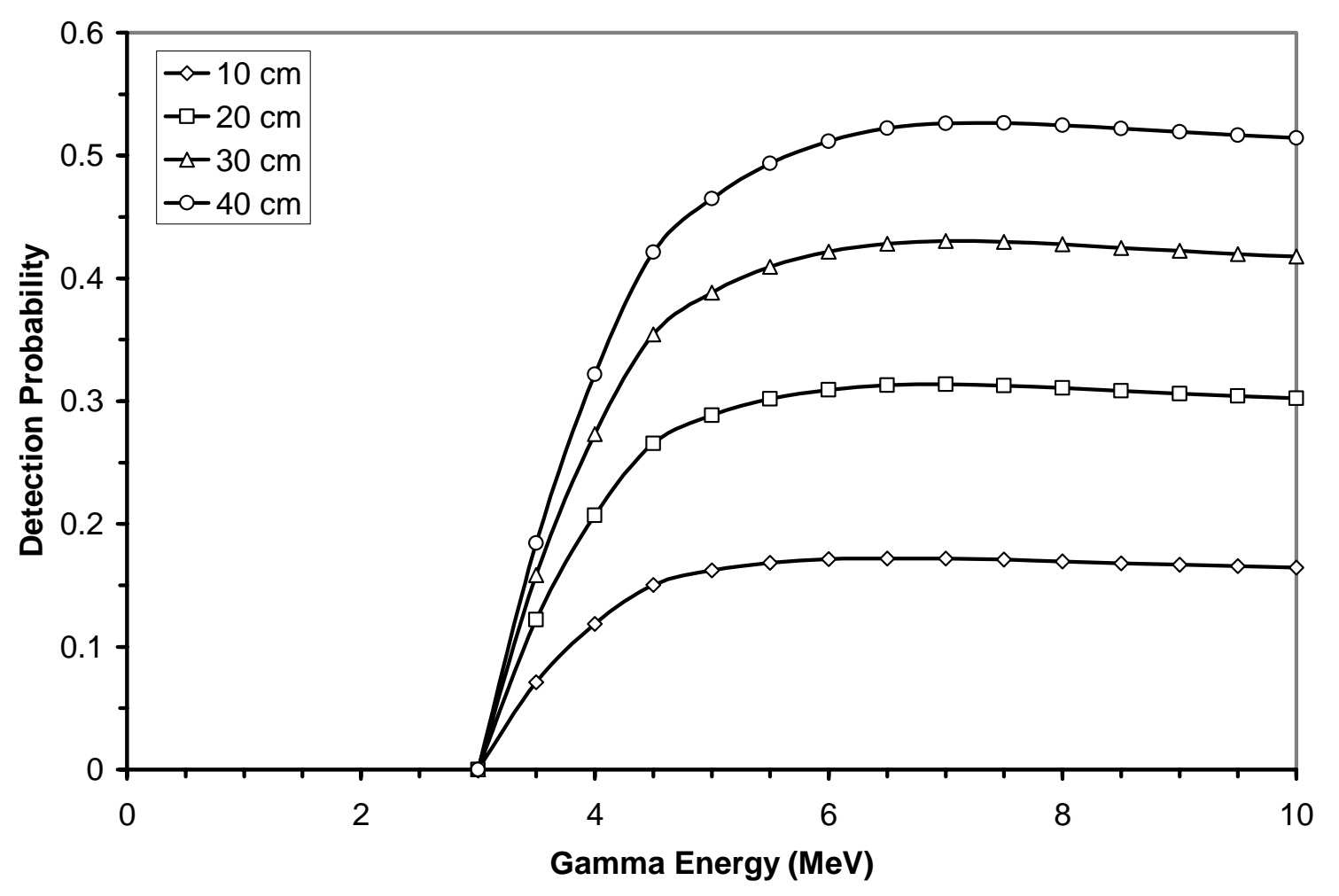

(c) 
Figure 11.

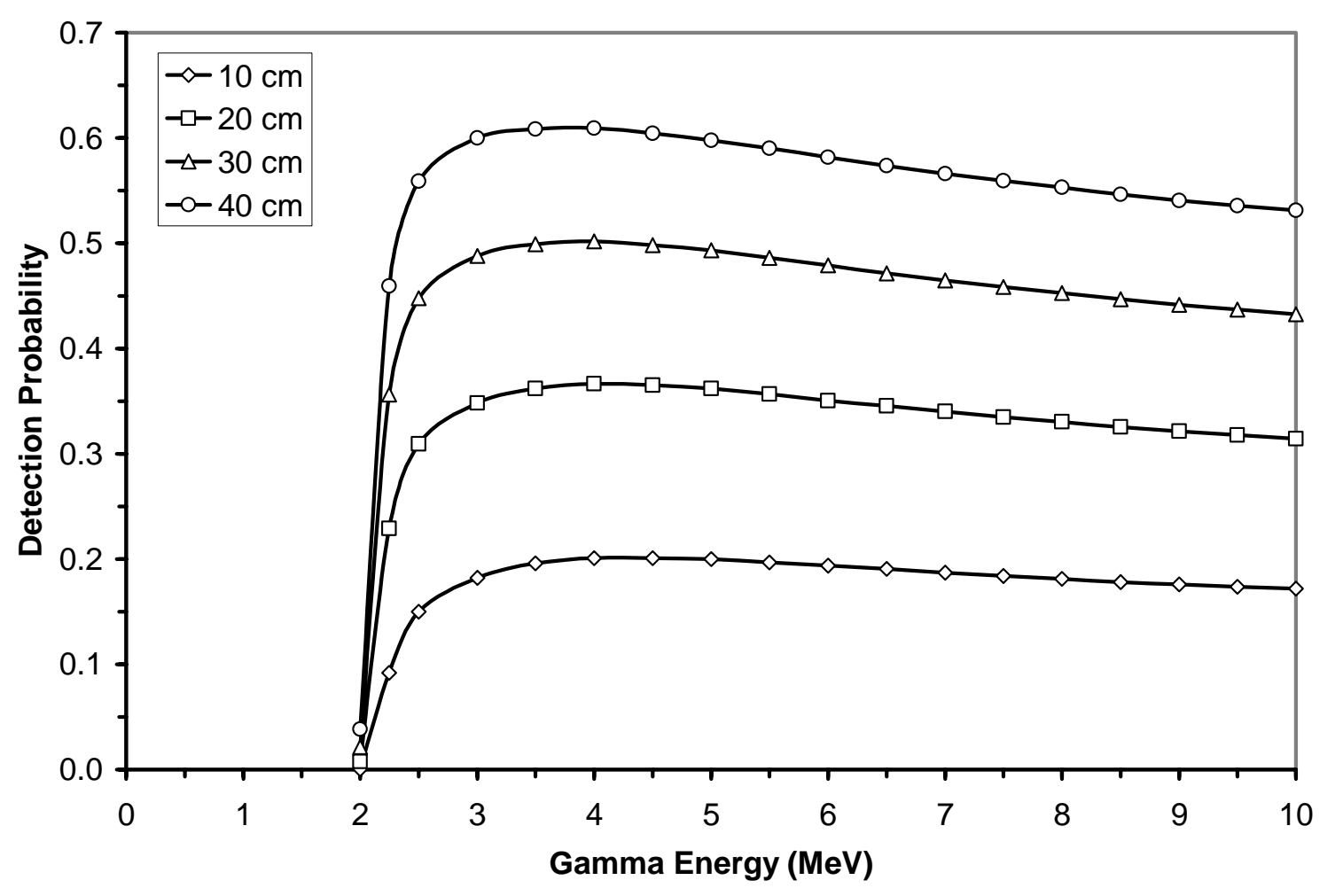

(a)

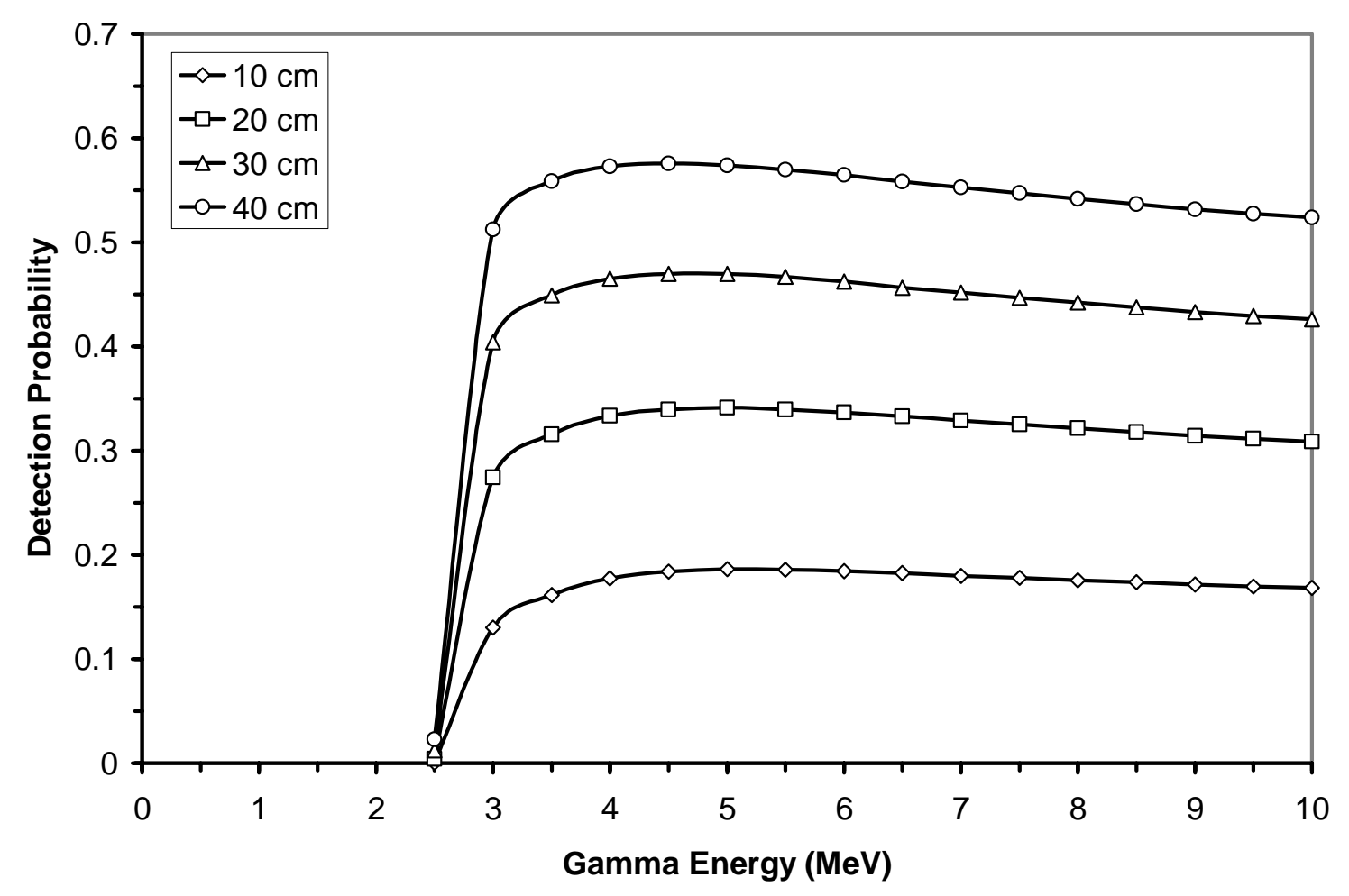

(b) 
Figure 11.

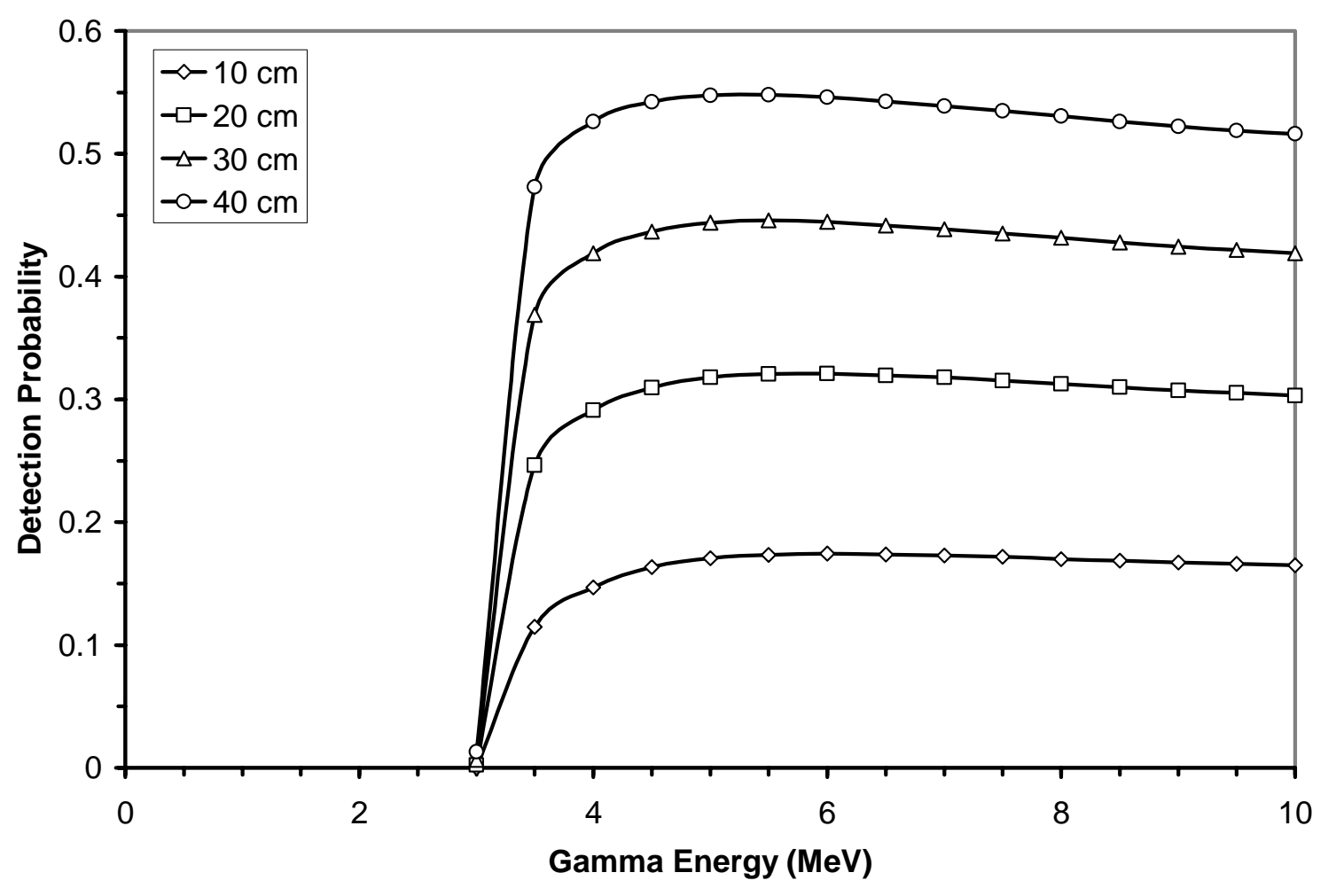

(c) 
Figure 12.

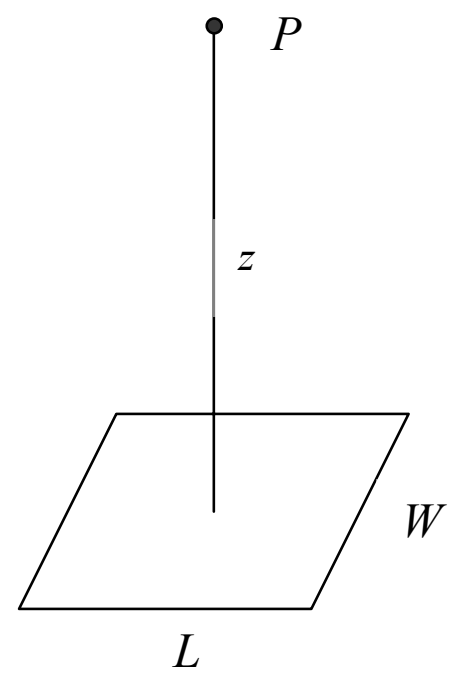


Figure 13.

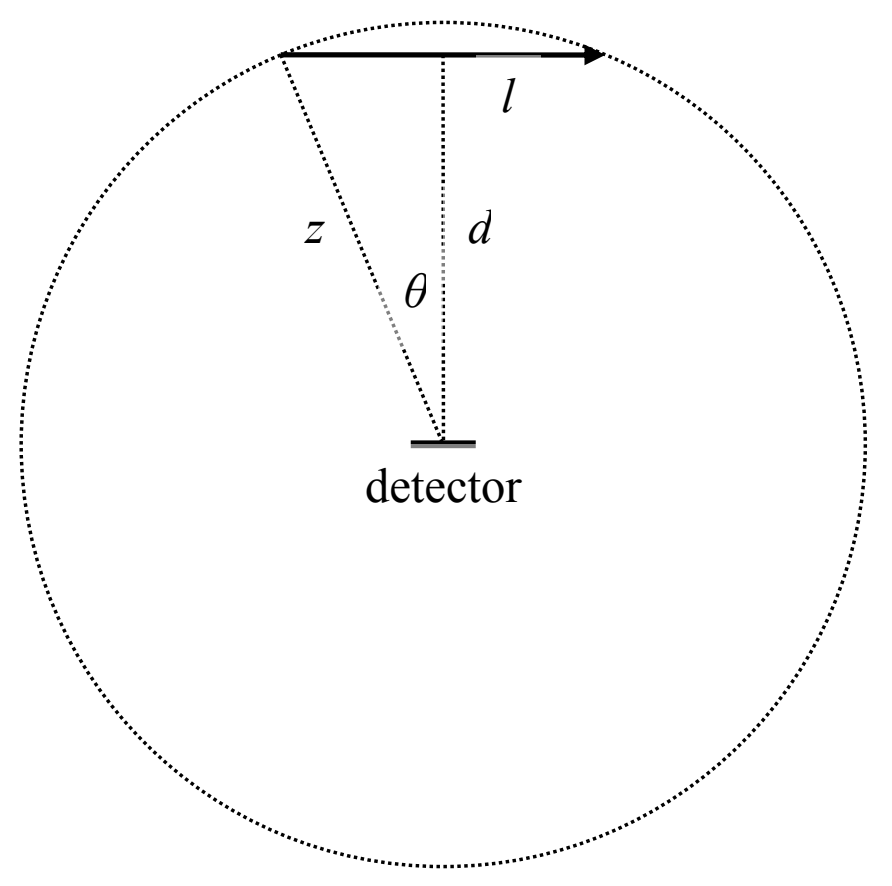


Figure 14.

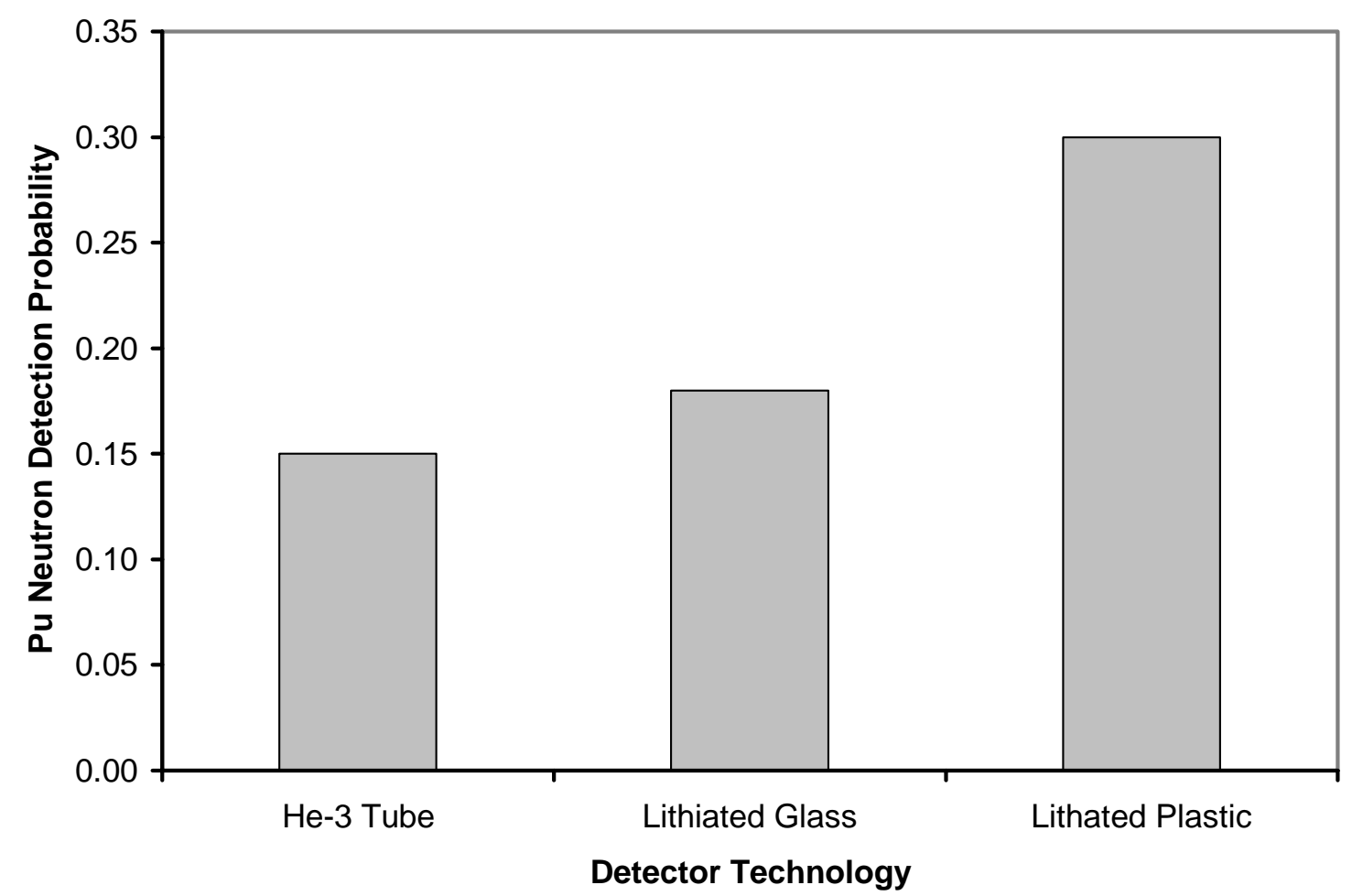




\section{Figure Captions}

Figure 1. Extruded plastic scintillator bar with wavelength-shifting (WLS) readout fibers. A pen is shown for scale.

Figure 2. Neutron detection probability for the following plastic scintillator thicknesses and lithium concentrations: (a) $10 \mathrm{~cm}, 0.1 \%$; (b) $10 \mathrm{~cm}, 0.5 \%$; (c) $10 \mathrm{~cm}, 2 \%$; (d) $20 \mathrm{~cm}$, $0.1 \%$; (e) $20 \mathrm{~cm}, 0.5 \%$; (f) $20 \mathrm{~cm}, 2 \%$; (g) $30 \mathrm{~cm}, 0.1 \%$; (h) $30 \mathrm{~cm}, 0.5 \%$; (i) $30 \mathrm{~cm}, 2 \%$.

Figure 3. Detection probability for $1.5 \mathrm{MeV}$ neutrons for various lithium concentrations and plastic scintillator thicknesses. Solid lines denote double time events and dashed lines denote the total neutron detection probability. Squares, diamonds, and triangles denote scintillator thicknesses of 10,20 , and $30 \mathrm{~cm}$, respectively.

Figure 4. Detection probability for $\mathrm{Pu}$ spontaneous fission neutrons for various lithium concentrations and plastic scintillator thicknesses. Solid lines denote double time events and dashed lines denote the total neutron detection probability. Squares, diamonds, and triangles denote scintillator thicknesses of 10,20 , and $30 \mathrm{~cm}$, respectively.

Figure 5. Distribution of neutron energies upon capture by ${ }^{6} \mathrm{Li}$. (Neutrons initially had 2 $\mathrm{MeV}$ of energy.) Squares, diamonds, and triangles denote lithium concentrations of $0.1 \%, 0.5 \%$, and $2 \%$, respectively.

Figure 6. Cumulative distribution function for time delay between initial scattering reaction by a fast neutron and its capture by ${ }^{6} \mathrm{Li}$ for various lithium concentrations in the scintillator. Neutrons initially had $2 \mathrm{MeV}$ of energy. Only data from neutrons whose scattering reactions produce measurable pulses (i.e. pulses that trip the LLD) are included.

Figure 7. Distribution of distances between the first scatter and neutron capture locations for neutrons with an initial energy of $2 \mathrm{MeV}$ in plastic scintillator thicknesses of (a) 10 $\mathrm{cm}$, (b) $20 \mathrm{~cm}$, and (c) $30 \mathrm{~cm}$.

Figure 8. Single interaction pulse amplitude gamma detection probabilities using various light emission intensity LLD values for scintillator thicknesses of (a) $10 \mathrm{~cm}$, (b) $20 \mathrm{~cm}$, (c) $30 \mathrm{~cm}$, and (d) $40 \mathrm{~cm}$.

Figure 9. Multiple interaction method (total pulse amplitude) gamma detection probabilities using various light emission intensity LLD values for scintillator thicknesses of (a) $10 \mathrm{~cm}$, (b) $20 \mathrm{~cm}$, (c) $30 \mathrm{~cm}$, and (d) $40 \mathrm{~cm}$.

Figure 10. Single interaction pulse amplitude gamma detection probability as a function of gamma energy when using count thresholds of (a) $2 \mathrm{MeVee}$, (b) $2.5 \mathrm{MeVee}$, and (c) 3 MeVee. 
Figure 11. Multiple interaction method (total pulse amplitude) gamma detection probability as a function of gamma energy when using count thresholds of (a) $2 \mathrm{MeVee}$, (b) $2.5 \mathrm{MeVee}$, and (c) $3 \mathrm{MeVee}$.

Figure 12. Configuration of a point $P$ located a distance $z$ from the center of a rectangular area $W \times L$ on a line perpendicular to the center of that area. For purposes of calculating stand-off detection distance, $P$ represents the source and $W \times L$ represents the detector.

Figure 13. Method used for determining the stand-off detection distance for the source in motion case. The value $z$ is what the stand-off distance for the source would be if it were stationary and a counting time equal to the time taken for the source to traverse the distance 1 were used. The value $d$ is the stand-off detection distance for the source when it is in motion.

Figure 14. Comparison of $\mathrm{Pu}$ spontaneous fission neutron detection probabilities for two state-of-the-art technologies with that of lithiated plastic. The double time event detection method for $20 \mathrm{~cm}$ plastic scintillator thickness with $0.5 \% \mathrm{Li}$ content is used. 
Table 1.

\begin{tabular}{|l|l|l|l|}
\hline & \multicolumn{3}{|c|}{ Detector size } \\
\hline Shielding & \multicolumn{1}{|c|}{$10 \mathrm{~m}^{2}$} & \multicolumn{1}{c|}{$1 \mathrm{~m}^{2}$} & \multicolumn{1}{c|}{$0.1 \mathrm{~m}^{2}$} \\
\hline None & $269 \mathrm{~m}$ & $151 \mathrm{~m}$ & $85 \mathrm{~m}$ \\
\hline Moderate & $190 \mathrm{~m}$ & $107 \mathrm{~m}$ & $60 \mathrm{~m}$ \\
\hline Heavy & $85 \mathrm{~m}$ & $48 \mathrm{~m}$ & $27 \mathrm{~m}$ \\
\hline
\end{tabular}

Table 1. Stand-off detection distances for the stationary source case with a one minute counting time. A neutron detection probability of 0.3 is used, corresponding to $20 \mathrm{~cm}$ lithiated plastic scintillator thickness with $0.5 \%$ Li concentration. 
Table 2.

\begin{tabular}{|l|l|l|l|}
\hline & \multicolumn{3}{|c|}{ Detector size } \\
\hline Shielding & \multicolumn{1}{|c|}{$10 \mathrm{~m}^{2}$} & $1 \mathrm{~m}^{2}$ & $0.1 \mathrm{~m}^{2}$ \\
\hline None & $261 \mathrm{~m} / 60 \mathrm{sec}$ & $136 \mathrm{~m} / 60 \mathrm{sec}$ & $63 \mathrm{~m} / 30 \mathrm{sec}$ \\
\hline Moderate & $178 \mathrm{~m} / 60 \mathrm{sec}$ & $86 \mathrm{~m} / 45 \mathrm{sec}$ & $40 \mathrm{~m} / 20 \mathrm{sec}$ \\
\hline Heavy & $63 \mathrm{~m} / 30 \mathrm{sec}$ & $29 \mathrm{~m} / 15 \mathrm{sec}$ & $13 \mathrm{~m} / 5 \mathrm{sec}$ \\
\hline
\end{tabular}

Table 2. Stand-off detection distances for the $8 \mathrm{kph}$ moving source case and their corresponding counting times (5 sec counting intervals were used). A neutron detection probability of 0.3 is used, corresponding to $20 \mathrm{~cm}$ lithiated plastic scintillator thickness with $0.5 \%$ Li concentration. 


\section{Table 3.}

\begin{tabular}{|l|l|c|}
\hline & \multicolumn{2}{|c|}{ Detector size } \\
\hline Shielding & \multicolumn{1}{|c|}{$10 \mathrm{~m}^{2}$} & $1 \mathrm{~m}^{2}$ \\
\hline None & $140 \mathrm{~m} / 10 \mathrm{sec}$ & $64 \mathrm{~m} / 5 \mathrm{sec}$ \\
\hline Moderate & $89 \mathrm{~m} / 5 \mathrm{sec}$ & $28 \mathrm{~m} / 5 \mathrm{sec}$ \\
\hline
\end{tabular}

Table 3. Stand-off detection distances for the $72 \mathrm{kph}$ moving source case and their corresponding counting times (5 sec counting intervals were used). A neutron detection probability of 0.3 is used, corresponding to $20 \mathrm{~cm}$ lithiated plastic scintillator thickness with $0.5 \%$ Li concentration. 